\title{
The diversity of methanol maser morphologies from VLBI observations *
}

\author{
A. Bartkiewicz ${ }^{1}$, M. Szymczak ${ }^{1}$, H. J. van Langevelde ${ }^{2,3}$, A. M. S. Richards ${ }^{4}$, and Y. M. Pihlström ${ }^{5,6}$ \\ 1 Toruń Centre for Astronomy, Nicolaus Copernicus University, Gagarina 11, 87-100 Toruń, Poland \\ e-mail: [annan;msz]@astro.uni.torun.pl \\ 2 Joint Institute for VLBI in Europe, Postbus 2, 7990 AA Dwingeloo, The Netherlands \\ e-mail: langevelde@jive.nl \\ 3 Sterrewacht Leiden, Postbus 9513, 2300 RA Leiden, The Netherlands \\ 4 Jodrell Bank Centre for Astrophysics, School of Physics and Astronomy, University of Manchester, M13 9PL, UK \\ e-mail: a.m.s.richards@manchester.ac.uk \\ 5 Department of Physics and Astronomy, MSC07 4220, University of New Mexico, Albuquerque, NM 87131, USA \\ e-mail: ylva@unm.edu \\ 6 National Radio Astronomy Observatory, 1003 Lopezville Road, Socorro, NM 87801, USA
}

Received 1 April 2009 / Accepted 23 April 2009

\begin{abstract}
Context. The $6.7 \mathrm{GHz}$ methanol maser marks an early stage of high-mass star formation, but the origin of this maser is currently a matter of debate. In particular it is unclear whether the maser emission arises in discs, outflows or behind shocks running into rotating molecular clouds.

Aims. We investigated which structures the methanol masers trace in the environment of high-mass protostar candidates by observing a homogenous sample of methanol masers selected from Torun surveys. We also probed their origins by looking for associated $\mathrm{H}$ II regions and IR emission.

Methods. We selected 30 methanol sources with improved position accuracies achieved using MERLIN and another 3 from the literature. We imaged 31 of these using the European VLBI Network's expanded array of telescopes with 5-cm (6-GHz) receivers. We used the VLA to search for $8.4 \mathrm{GHz}$ radio continuum counterparts and inspected Spitzer GLIMPSE data at 3.6-8 $\mu \mathrm{m}$ from the archive.

Results. High angular resolution images allowed us to analyze the morphology and kinematics of the methanol masers in great detail and verify their association with radio continuum and mid-infrared emission. A new class of "ring-like" methanol masers in starforming regions appeared to be suprisingly common, $29 \%$ of the sample.

Conclusions. The new morphology strongly suggests that methanol masers originate in the disc or torus around a proto- or a young massive star. However, the maser kinematics indicate the strong influence of outflow or infall. This suggests that they form at the interface between the disc/torus and a flow. This is also strongly supported by Spitzer results because the majority of the masers coincide with $4.5 \mu \mathrm{m}$ emission to within less than $1^{\prime \prime}$. Only four masers are associated with the central parts of UC H II regions. This implies that $6.7 \mathrm{GHz}$ methanol maser emission occurs before $\mathrm{H} \mathrm{II} \mathrm{region} \mathrm{observable} \mathrm{at} \mathrm{cm}$ wavelengths is formed.
\end{abstract}

Key words. stars: formation - ISM: molecules - masers - instrumentation: high angular resolution

\section{Introduction}

Methanol masers are commonly assumed to be associated with the environments of high-mass protostars, which provide the conditions required for methanol first to form on grains and then to be sublimated off, and finally to excite the maser transitions (Dartois et al. 1999; Cragg et al. 2002). Methanol maser emission at $6668.519 \mathrm{MHz}$ is one of the strongest and most widespread (Menten 1991) of the the first observable manifestations of a newly formed high-mass star. Emission towards the archetypical source $\mathrm{W} 3(\mathrm{OH})$ is characterized with a brightness temperature of up to $\sim 3 \times 10^{12} \mathrm{~K}$ from a spot of intrinsic size of 0.'0014 (Menten et al. 1992). The gas environment of even distant ( $>5 \mathrm{kpc}$ ) high-mass stars can thus be probed on scales as

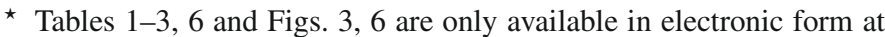
http://www. aanda.org small as $\sim 5$ AU when observed with milliarcsecond (mas) resolution using Very Long Baseline Interferometry (VLBI).

All major methanol targeted surveys of high angular resolution taken to date are summarized in Table 1 . This indicates the diversity of the sample selections and observing parameters, which might have affected the data interpretation.

The first observations of the $6.7 \mathrm{GHz}$ maser line at arcsec resolution, using the ATCA, concentrated on the brightest sources (Norris et al. 1993), followed by more extensive surveys (Phillips et al.1998; Walsh et al.1998). The relative positions of individual maser spots were determined with $\sim 0$ ' $^{\prime} 05$ accuracy and the distribution of bright $\left(>0.5 \mathrm{Jy} \mathrm{beam}^{-1}\right)$ maser spots was resolved for the majority of targets. Various morphological structures, such as simple, linear, curved, complex and double, were found. The linear sizes varied between 190 and 5600 AU. Norris et al. (1993) found that in 10 out of the 15 sources imaged the masers are located along lines or arcs of which five sources 
show a clear velocity gradient along the line. They proposed that the linear structures with velocity gradients are produced by the masers residing in rotating discs seen edge-on. Phillips et al. (1998) increased the sample of masers studied with the ATCA to 45 objects, finding that 17 of them show morphologies and monotonic velocity gradients consistent with the circumstellar disc hypothesis. Assuming a Keplerian disc, the enclosed masses range from 1 to $75 M_{\odot}$. In a sample of 97 sources Walsh et al. (1998) found 36 masers with some linear structure but this was clearly-defined for only 9 sources. Therefore, the Keplerian disc hypothesis accounts for only a small proportion of their sources; most maser sites do not exhibit a systematic velocity gradient. They suggested that the masers form rather behind shock fronts.

The methanol masers are often not associated with detectable continuum emission at centimeter wavelengths. Twenty-five of sources in the sample of Phillips et al. (1998) are associated with an ultra-compact H II (UCHII) region wherein the methanol masers are slightly offset from the peak continuum emission. They argued that the methanol sources without an H II region are possibly associated with less massive stars than those with coincident radio continuum emission. Walsh et al. (1998) also found that most of their maser sources are not associated with radio continuum brighter than $\sim 1 \mathrm{mJy}$, implying that the phase of methanol maser occurs before an observable UCH II region is formed. This suggestion was confirmed for another sample of high-mass protostellar candidates (Beuther et al. 2002).

The detailed spatial structure of the methanol maser emission in these sources should provide further clues to their origin. To date, only a few observations at mas resolution have been published. Minier et al. (2000) observed 14 bright sources with the EVN. In 10 targets they found elongated structures with linear velocity gradients, which can be interpreted in terms of a circumstellar edge-on disc model. However, the estimates of central mass with this model for all but one source seemed to be far lower than expected for a high-mass star. Minier et al. (2000) suggested that this could be because the detectable masers delineate only part of the full diameters of the discs. They also proposed other models, such as accelerating outflows and shock fronts. Dodson et al. (2004) used the LBA to image five maser sites with linear morphologies at arcsecond resolutions. Their milliarcsecond resolution data were interpreted using a model of an externally generated planar shock propagating through a rotating dense molecular clump or star-forming core.

Van der Walt et al. (2007) argued that the model of Dodson et al. is inconsistent with the observed kinematic properties of the masers. They concluded that the observed rest frame distribution of maser velocities can be reproduced well with a simple Keplerian-like disc model. Source NGC 7538 IRS 1 is understood to be a good example of an edge-on Keplerian disc (Minier et al. 2000; Pestalozzi et al. 2004). However, high angular resolution mid-infrared (MIR) data were used to demonstrate that the outflow scenario is also plausible since the maser is not oriented perpendicular to the outflow as expected (De Buizer $\&$ Minier 2005). The kinematic and spatial distribution of the $12 \mathrm{GHz}$ methanol masers in $\mathrm{W} 3(\mathrm{OH})$ were successfully fitted by a model of a conical bipolar outflow (Moscadelli et al. 2002).

The initial methanol imaging surveys were mostly of relatively low (arcsec) resolution, whilst very long baseline interferometer (VLBI) studies probably missed fainter emission, such as from the edges or the far side of putative discs. For the first time, we have studied a large sample, detecting 31 sources at mas resolution and $\sim 10 \mathrm{mJy}$ sensitivity, with sufficient astrometric precision to complete robust identifications. This enables us to test the competing hypotheses for the origins of methanol
6.7 GHz maser emission, namely circumstellar discs, outflows, or propagating shock fronts. In this paper, we present $\mathrm{EVN}^{1}$ observations of the methanol line and VLA observations of continuum emission for a homogeneous sample of the methanol masers discovered in the Torun untargeted survey (Szymczak et al. 2000, 2002). The preliminary results of methanol observations were partly published in Bartkiewicz et al. (2004, 2006, 2009). As part of this survey the discovery of a ring structure in G23.657-00.127 was reported by Bartkiewicz et al. (2005). Our observations have enabled us to detect a wide diversity of methanol maser geometries and demonstrate for the first time that in a large fraction of sources the distribution of the spots is ring-like.

\section{Observations and data reduction}

\subsection{Sample selection}

The sources were selected from two previous samples obtained using the Torun $32 \mathrm{~m}$ antenna: the blind survey of the $6.7 \mathrm{GHz}$ methanol maser line (Szymczak et al. 2002) and the methanol survey of IRAS-selected objects (Szymczak et al. 2000). The untargeted flux-limited $\left(3 \sigma_{\mathrm{rms}} \simeq 1.6 \mathrm{Jy}\right)$ complete survey of the Galactic plane region $20^{\circ} \leq l \leq 40^{\circ}$ and $|b| \leq 0$. 52 enabled the detection of 100 sources of which 26 were new. The same field includes 22 sources discovered in the earlier survey of IRAS-selected objects. These 48 objects were chosen as a sample for detailed studies. We note that the mean single-dish methanol maser flux density of these 48 sources is $16 \mathrm{Jy}$, a factor of 2 lower than that of the rest sources in the original samples. This may have introduced a selection effect for masers that are more distant, have intrinsically weaker maser emission, or are less aligned with the line of sight.

Depending on the maser flux densities the source coordinates obtained using the $32 \mathrm{~m}$ dish are accurate to within $25-70^{\prime \prime}$. We undertook astrometric measurements using the first two MERLIN ${ }^{2}$ antennas to be equipped with 6.7-GHz receivers (Mark II and Cambridge). These single baseline observations detected 30 of the 48 objects, providing positions with subarcsecond accuracy (see Sect. 2.2). We included three additional objects in the same region that had not been detected in the Torun surveys, for which accurate positions are reported in the literature. These are: G22.357+00.066 (Walsh et al. 1998), G25.411+00.105, and G32.992+00.034 (Beuther et al. 2002). The total sample selected for VLBI observations comprised 33 sources in the Galactic within the region defined by $21.4^{\circ} \leq$ $l \leq 39.1^{\circ}$ and $-0.38^{\circ} \leq b \leq 0.56^{\circ}$ (Table 2 ).

\subsection{MERLIN astrometry}

The MERLIN observations at a rest frame frequency of $6668.519 \mathrm{MHz}$ were carried out during observing runs between 2002 May and June and 2003 March and May. The typical onsource observing time was about $1 \mathrm{~h}$ for each target, and frequent observations of nearby phase reference sources and other calibrators were completed. Standard single-baseline data reduction procedures were applied (Diamond et al. 2003) using AIPS (the Astronomical Image Processing System). We searched for emission from each target in its vector-averaged spectrum by shifting the phase center from $-200^{\prime \prime}$ to $+200^{\prime \prime}$ in right ascension and from $-500^{\prime \prime}$ to $+500^{\prime \prime}$ in declination (at $1^{\prime \prime}$ intervals) to locate

\footnotetext{
1 The European VLBI Network.

2 The Multi-Element Radio Linked Interferometer Network.
} 
the position giving the maximum intensity for the main maser feature. We simultaneously inspected the phase, which should be close to $0^{\circ}$ at this position in the spectral channels containing the main feature. Finally, we produced a large $\left(40^{\prime \prime} \times 40^{\prime \prime}\right)$ dirty map of the main feature for the channel of the highest spectral signalto-noise ratio, centered on the estimated position. The brightest spot was then assumed to be as the maser position. A typical beam was $200 \times 20 \mathrm{mas}^{2}$ at a position angle of $20^{\circ}$. Because of the very poor $u v$-coverage, we were unable to derive the maser structures.

Methanol maser emission was detected towards 30 of the 48 sources observed, giving absolute positions of sufficient accuracy for follow-up EVN observations. The MERLIN singlebaseline astrometric accuracy was between 0.3 and $1^{\prime \prime}$ in most cases, depending on the source brightness. However, the absolute position uncertainty of sources at $\mid$ Dec. $\mid<3.5$ increased to $5-10^{\prime \prime}$. The mean differences between the coordinates obtained using a single dish and using the MERLIN single baseline were $30^{\prime \prime} \pm 6^{\prime \prime}$ and $20^{\prime \prime} \pm 4^{\prime \prime}$ in right ascension and declination, respectively. No emission was detected towards the remaining 18 sources above a sensitvity limit of 0.3 Jy (Table 3 ). The possible causes of non-detection are: variability, large errors in the single dish positions, interference (for a few targets), or extended emission, resolved out by the interferometer.

\subsection{EVN observations}

The EVN observations of 33 targets in the $6.7 \mathrm{GHz}$ methanol maser line were carried out in seven observing runs between 2003 and 2007 (projects EN001, EN003, EB031, EB034). The observing parameters are summarized in Table 4 including the date, duration of each run, working antennas, cycle time between the maser and phase-calibrator, spectral resolution, typical synthesized beam size, and $1 \sigma_{\text {rms }}$ noise level in a spectral channel.

Each observing run included scans of 3C 345, which was adopted as a bandpass, delay and rate calibrator. Five or six sources were, typically, observed in each run, selected to be within a few degrees of each other in projection on the sky and of similar maser emission velocities. A phase-referencing scheme was applied in which a nearby, sufficiently bright phasecalibrator for each session was selected from the VLBA list. Details are given in Table 2. We used a spectral bandwidth of $2 \mathrm{MHz}$ yielding a velocity coverage of $\sim 100 \mathrm{~km} \mathrm{~s}^{-1}$. In all sessions, the Mk V recording system was used with the exception of the first epoch when data were recorded on tapes (Mk IV system). The data were correlated with the Mk IV Data Processor operated by JIVE with 1024 spectral channels. In 4a and 4b runs only, when all nine antennas were operating, data were correlated with 512 spectral channels in two passes i.e., separately for LHC and RHC polarization, because of the correlator limitations. Left- and right-hand circular polarization data were averaged to increase the signal-to-noise ratio.

\subsubsection{Calibration and imaging}

The data calibration and reduction were carried out in AIPS, employing standard procedures for spectral line observations. First, the amplitude was calibrated using measured antenna gain curves and system temperatures. In the second step, the parallactic angle corrections were added. The Effelsberg antenna was used as a reference when calibrating the data from all sessions. The instrumental delays for calibrated using phase-calibrator and 3C 345 observations. The phase-calibrator was mapped and a few iterations of self-calibration were completed, gradually shortening the time interval from $120 \mathrm{~min}$ to $1 \mathrm{~min}$. Flux densities of 240, 202, 80, and $237 \mathrm{mJy}$ were obtained for phase-calibrators J1825-0737, J1834-0301, J1856+0610, and $\mathrm{J} 1907+0127$, respectively. The maser data were corrected for the effects of the Earth's rotation and its motion within the Solar System and towards the LSR. After applying all corrections from the calibration sources, we compiled preliminary maps of the channel containing the brightest and most compact peak. We then used the clean components of that map, if possible, as the starting model for further rounds of self-calibration. In two cases, high quality images could only be obtained after the first round of self-calibration using a default model at the pointing position.

We searched for emission using large $\left(2^{\prime \prime} \times 2^{\prime \prime}\right)$ maps over the entire band. We then created naturally-weighted $0.5^{\prime \prime} \times 0.5^{\prime \prime}$ cleaned images to use in analyzing maser properties. The beam sizes for each data set are listed in Table 4. The pixel separation was 1 mas. The rms noise levels in line-free channels were typically between 4 and $10 \mathrm{mJy}^{\text {beam }}{ }^{-1}$ depending on the run. The positions of all maser spots (above $5 \sigma_{\text {rms }}$ ) in each individual channel map were determined by fitting two-dimensional Gaussian components. The formal fitting errors were, typically, $0.01-0.15$ mas in right ascension and $0.02-0.5$ mas in declination, depending on the source strength and structure.

The astrometric accuracy for the 29 sources with phasereferenced maps is limited by four factors. Firstly, the phasereference source positions have an accuracy of $<1.5$ mas. Secondly, the antenna positions have an accuracy of $\sim 1 \mathrm{~cm}$, corresponding to an uncertainty of $\sim 1$ mas in RA and 2-3 mas in Dec. Thirdly, the separations between the targets and phase reference sources were $\lesssim 4.5$, which translate into a potential phase solution transfer error equivalent to 2 mas in RA and 4-5 mas in Dec. Fourthly, the position uncertainty due to noise is given by (beamsize)/(signal-to-noise ratio), which is $\ll 1$ mas for all our reference features. This infers a total astrometric uncertainty of 3 mas in RA and 6 mas in Dec. For the remaining four sources, we were unable to improve on the original MERLIN positions.

\subsection{VLA continuum observations}

In order to investigate the presence, position, and distribution of radio continuum emission associated with the $6.7 \mathrm{GHz}$ methanol maser emission, we used the VLA at $8.4 \mathrm{GHz}$ in A configuration (the project AB1250). Data were taken on 2007 August 18 for $12 \mathrm{~h}$ in a standard VLA continuum mode towards 30 sources in the sample. We did not observe the three sources that had not been included in the Torun surveys. We used 3C 286 as a flux calibrator and two phase-calibrators, 18517+0355 and 18323-1035, from a standard VLA list. To increase signal-tonoise ratio, we employed the fast switching mode with a cycle time between the phase-calibrator and the target of $50 \mathrm{~s}+250 \mathrm{~s}$. This sequence lasted for $20 \mathrm{~min}$ for each target.

The data reduction was carried out following to the standard recipies from AIPS Cookbook Appendix A (NRAO 2007). The amplitude and phases of 3C 286 were corrected using the default source model and 3C 286 was then used to find the phasecalibrator flux densities. The antenna gains were calibrated using the phase-calibrator data. Some bad points were flagged and finally the images were created with natural weighting. The $1 \sigma_{\text {rms }}$ noise level in the maps was typically $\sim 50 \mu \mathrm{Jy} \mathrm{beam}^{-1}$ and the beam was $0 . ! 35 \times 0.25$. 
Table 4. Details of EVN observations.

\begin{tabular}{|c|c|c|c|c|c|c|c|}
\hline $\begin{array}{l}\text { Observing } \\
\text { run }\end{array}$ & Date & $\begin{array}{c}\text { Duration } \\
\text { (h) }\end{array}$ & Telescopes* & $\begin{array}{c}\text { Cycle time } \\
(\mathrm{min})\end{array}$ & $\begin{array}{c}\text { Spectral channel } \\
\text { separation } \\
\left(\mathrm{km} \mathrm{s}^{-1}\right)\end{array}$ & $\begin{array}{c}\text { Synthesized } \\
\text { beam }(\alpha \times \delta ; \mathrm{PA}) \\
\left(\operatorname{mas} \times \operatorname{mas} ;{ }^{\circ}\right)\end{array}$ & $\begin{array}{c}\text { Rms noise } \\
\text { per channel } \\
\left(\mathrm{mJy} \mathrm{beam}^{-1}\right)\end{array}$ \\
\hline 1 & 2003 Jun. 08 & 12 & CmJbEfOn & $6.00+4.00$ & 0.09 & $6 \times 16 ;-1$ & 10 \\
\hline 2 & 2004 Nov. 11 & 12 & $\mathrm{CmDaEfMcNtOnTrWb}$ & $3.75+1.75$ & 0.09 & $6 \times 16 ;+7$ & 4 \\
\hline $3 \mathrm{a}$ & 2006 Feb. 22 & 10 & CmJbEfHhMcNtOnWb & $3.75+1.75$ & 0.09 & $5 \times 15 ;+31$ & 10 \\
\hline $3 b$ & 2006 Feb. 23 & 10 & CmJbEfHhMcOnWb & $3.75+1.75$ & 0.09 & $6 \times 14 ;+34$ & 7 \\
\hline $4 a$ & 2007 Jun. 13 & 10 & CmJbEfHhMcNtOnTrWb & $3.25+1.75$ & 0.18 & $6 \times 12 ;+20$ & 4 \\
\hline $4 \mathrm{~b}$ & 2007 Jun. 14 & 10 & CmJbEfHhMcNtOnTrWb & $3.25+1.75$ & 0.18 & $6 \times 11 ;+25$ & 4 \\
\hline $4 \mathrm{c}$ & 2007 Jun. 15 & 10 & CmJbEfMcNtOnTrWb & $3.25+1.75$ & 0.09 & $6 \times 13 ;+35$ & 6 \\
\hline
\end{tabular}

* Cm-Cambridge, Da-Darnhall, Jb-Jodrell Bank, Ef-Effelsberg, Hh-Hartebeesthoek, Mc-Medicina, Nt-Noto, On-Onsala, Tr-Toruń, Wb-Westerbork.

Table 5. Results of EVN observations.

\begin{tabular}{|c|c|c|c|c|c|c|c|c|c|}
\hline \multirow{2}{*}{$\begin{array}{l}\text { Source } \\
\text { Gll.1ll } \pm \text { bb.bbb }\end{array}$} & \multicolumn{2}{|c|}{ Position $(\mathrm{J} 2000)$} & \multirow{2}{*}{$\begin{array}{c}\begin{array}{c}V_{\mathrm{p}} \\
\left(\mathrm{km} \mathrm{s}^{-1}\right)\end{array} \\
\end{array}$} & \multirow{2}{*}{$\begin{array}{c}\Delta V \\
\left(\mathrm{~km} \mathrm{~s}^{-1}\right) \\
\end{array}$} & \multirow{2}{*}{$\begin{array}{c}S_{\mathrm{p}} \\
\left.\text { (Jy beam }^{-1}\right)\end{array}$} & \multicolumn{2}{|l|}{ Area } & \multirow[t]{2}{*}{ Class $^{* *}$} & \multirow{2}{*}{$\begin{array}{c}\Delta_{\mathrm{MIR}} \\
\left({ }^{\prime \prime}\right) \\
\end{array}$} \\
\hline & $\mathrm{RA}(\mathrm{h} \mathrm{m} \mathrm{s})$ & Dec. $\left({ }^{\circ}{ }^{\prime \prime \prime} \prime\right)$ & & & & $($ mas $\times$ mas $)$ & $\mathrm{PA}\left({ }^{\circ}\right)$ & & \\
\hline G21.407-00.254 & 183106.33794 & -102137.4108 & 89.0 & 3.00 & 2.76 & $138 \times 39$ & -87 & $\overline{\mathrm{C}}$ & 0.23 \\
\hline G22.335-00.155 & 183229.40704 & -09 2929.6840 & 35.6 & 3.10 & 1.71 & $49 \times 11$ & +16 & $\mathrm{~L}$ & 0.67 \\
\hline G22.357+00.066 & 183144.12055 & -092212.3129 & 79.7 & 9.20 & 10.54 & $330 \times 174$ & -5 & $\mathrm{C}$ & 0.51 \\
\hline G23.207-00.377 & 183455.21212 & -084914.8926 & 77.1 & 13.20 & 9.30 & $313 \times 255$ & -69 & $\mathrm{R}$ & 0.56 \\
\hline G23.389+00.185 & 183314.32477 & -082357.4723 & 75.4 & 6.00 & 21.55 & $205 \times 134$ & +59 & $\mathrm{R}$ & 0.16 \\
\hline G23.657-00.127 & 183451.56482 & -081821.3045 & 82.6 & 10.80 & 3.62 & $351 \times 345$ & -82 & $\mathrm{R}$ & 0.50 \\
\hline G23.707-00.198 & 183512.36600 & -081739.3577 & 79.2 & 23.30 & 6.06 & $130 \times 110$ & -83 & A & 0.74 \\
\hline G23.966-00.109 & 183522.21469 & -080122.4698 & 70.9 & 4.20 & 5.47 & $35 \times 4$ & -45 & $\mathrm{~L}$ & 0.19 \\
\hline G24.148-00.009 & 183520.94266 & -074855.6745 & 17.8 & 1.40 & 3.60 & $28 \times 3$ & -11 & $\mathrm{~L}$ & 0.16 \\
\hline $\mathrm{G} 24.541+00.312$ & 183455.72152 & -071906.6504 & 105.7 & 6.80 & 7.75 & $137 \times 53$ & +78 & A & 0.45 \\
\hline G24.634-00.324 & 183722.71271 & -073142.1439 & 35.4 & 13.40 & 3.03 & $73 \times 21$ & -60 & $\mathrm{R}$ & 1.01 \\
\hline G25.411+00.105 & 183716.92106 & -063830.5017 & 97.3 & 5.20 & 3.43 & $225 \times 162$ & +79 & $\mathrm{R}$ & 0.64 \\
\hline G26.598-00.024 & 183955.92567 & -053844.6424 & 24.2 & 3.30 & 3.04 & $361 \times 152$ & -76 & $\mathrm{R}$ & 0.39 \\
\hline G27.221+00.136 & 184030.54608 & -050105.3947 & 118.8 & 16.10 & 12.54 & $104 \times 79$ & +6 & $\mathrm{C}$ & 0.89 \\
\hline G28.817+00.365 & 184237.34797 & -032940.9216 & 90.7 & 5.20 & 3.14 & $115 \times 28$ & +45 & $\mathrm{~A} / \mathrm{R}$ & 4.71 \\
\hline G30.318+00.070 & 184625.02621 & -021740.7539 & 36.1 & 1.90 & 0.52 & $50 \times 6$ & -50 & $\mathrm{~L}$ & 0.87 \\
\hline G30.400-00.296 & 184752.29976 & -022316.0539 & 98.5 & 6.70 & 2.77 & $199 \times 97$ & +47 & $\mathrm{C} / \mathrm{R}$ & 2.28 \\
\hline G31.047+00.356 & 184643.85506 & -013054.1551 & 80.7 & 6.30 & 1.99 & $68 \times 27$ & +72 & $\mathrm{R}$ & 1.73 \\
\hline G31.156+00.045* & 184802.347 & -013335.095 & & & & & & & 6.62 \\
\hline G31.581+00.077 & 184841.94108 & -011002.5281 & 95.6 & 4.80 & 2.72 & $217 \times 105$ & +79 & $\mathrm{~A} / \mathrm{R}$ & 4.23 \\
\hline G32.992+00.034 & 185125.58288 & +00 0408.3330 & 91.8 & 5.20 & 6.21 & $115 \times 68$ & -80 & $\mathrm{C}$ & 1.48 \\
\hline G33.641-00.228* & 185332.563 & +003139.180 & 58.8 & 5.30 & 28.3 & $167 \times 61$ & +66 & A & 1.22 \\
\hline G33.980-00.019 & 185325.01833 & +00 5525.9760 & 58.9 & 6.90 & 3.78 & $89 \times 43$ & +82 & $\mathrm{R}$ & 0.95 \\
\hline G34.751-00.093 & 185505.22296 & +013436.2612 & 52.7 & 3.10 & 1.95 & $49 \times 11$ & -56 & $\mathrm{R}$ & 0.47 \\
\hline G35.793-00.175* & 185716.894 & +02 2757.910 & 60.7 & 2.80 & 9.70 & $10 \times 2$ & +65 & $\mathrm{~L}$ & 1.12 \\
\hline G36.115+00.552 & 185516.79345 & +030505.4140 & 73.0 & 14.80 & 11.74 & $1201 \times 297$ & -79 & $\mathrm{P}$ & 2.42 \\
\hline G36.705+00.096 & 185759.12288 & +032406.1124 & 53.1 & 10.60 & 7.58 & $64 \times 18$ & -16 & $\mathrm{C}$ & 0.32 \\
\hline G37.030-00.039 & 185903.64233 & +033745.0861 & 78.6 & 0.70 & 0.69 & $2 \times 1$ & -15 & S & 0.61 \\
\hline G37.479-00.105* & 190007.145 & +035953.350 & & & & & & & 1.73 \\
\hline G37.598+00.425 & 185826.79772 & +042045.4570 & 85.8 & 4.50 & 3.91 & $94 \times 28$ & +87 & $\mathrm{C}$ & 1.24 \\
\hline G38.038-00.300 & 190150.46947 & +042418.9559 & 55.7 & 4.20 & 2.17 & $31 \times 23$ & +10 & $\mathrm{C}$ & 0.28 \\
\hline G38.203-00.067 & 190118.73235 & +043934.2938 & 79.6 & 6.00 & 0.83 & $182 \times 58$ & -44 & $\mathrm{C}$ & 1.74 \\
\hline G39.100+00.491 & 190058.04036 & +054243.9214 & 15.3 & 3.30 & 2.07 & $183 \times 37$ & +52 & $\mathrm{C}$ & 0.82 \\
\hline
\end{tabular}

* Coordinates derived from the single MERLIN baseline data.

** Class of morphology as described in Sect. 3.3: $\mathrm{S}$ - simple, L - linear, $\mathrm{R}$ - ring, C - complex, A - arched, P - pair.

\section{Results}

\subsection{Maser emission}

We successfully mapped a total of 31 out of 33 methanol masers observed with the EVN. We were unable to image G31.156+00.045 and G37.479-00.105 because of the weakness $(<30 \mathrm{mJy})$ of the emission and to strong spike artefacts in the channels at the maser velocity, respectively. We were unable to improve on the MERLIN astrometry for G33.641-00.228 and G35.793-00.175 due to a problem with the EVN phasereferencing that appeared during the first observing run. We created fringe rate maps of the brightest channels of the targets but still failed to determine the absolute position of these two sources. The target sources were near zero declination (from +0.5 to +2.5 ). Furthermore, because of the use of only four EVN telescopes, the $u v$-plane coverage was poor for $\mathrm{N}-\mathrm{S}$ baselines. It is probable that these factors together with a too long phasereferencing cycle time precluded a proper phase calibration. The position of the third source, G36.115+00.552, observed in the first run, was easily ascertained during the $4 \mathrm{c}$ run when eight antennas were working and the cycle time between the maser and phase-calibrator was shorter.

The results are summarized in Table 5. The names of the maser sources correspond to the Galactic coordinates of the 
brightest spot of each target. The absolute coordinates, the LSR velocity $\left(V_{\mathrm{p}}\right)$, and the intensity $\left(S_{\mathrm{p}}\right)$ are given for the brightest spot of each target. We also indicate the velocity range of emission $\Delta V$. The area containing all maser emission from each source was parameterised by measuring the extent of the maser emission along the line given by a least squares fit to the maser spot distribution (major axis and position angle) and in the perpendicular direction (minor axis). The morphological class based on the relative positions of methanol maser spots and the angular separation $\Delta_{\mathrm{MIR}}$ of the brightest spot of each source from the nearest $4.5 \mu \mathrm{m}$ source (see Sect. 4.2) are also given in Table 5.

In Fig. 1, we present the spectra and distribution of the methanol maser emission for the 31 imaged targets. The spectra were extracted from the map datacubes using the AIPS task ISPEC. They represent the total amount of emission seen in the maps. In order to display the detailed structures of masers, we show all the spots detected in each of the individual channel maps. If spots appear at the same positions within half of the beamwidth in at least three or two consecutive channels, for observations with a spectral resolution of $0.09 \mathrm{~km} \mathrm{~s}^{-1}$ and $0.18 \mathrm{~km} \mathrm{~s}^{-1}$, respectively, we refer to them as a cluster. The relevant parameters of all maser clusters for each source are listed in Table 6: the position ( $\triangle \mathrm{RA}, \Delta \mathrm{Dec}$.) relative to the brightest spot (given in Table 5), the peak intensity $\left(S_{\mathrm{p}}\right)$, and the LSR velocity $\left(V_{\text {LSR }}\right)$ of the brightest spot within a cluster. The velocity fullwidth at half-maximum ( $F W H M)$ and the fitted peak amplitude $\left(S_{\text {amp }}\right)$ are given if the spectrum of the cluster has a Gaussian profile.

\subsection{Radio continuum emission}

We detected $8.4 \mathrm{GHz}$ continuum emission in eight of the fields centered on methanol masers. Table 7 lists the continuum source names (derived from the Galactic coordinates of the $8.4-\mathrm{GHz}$ peak fluxes), the peak and the integrated intensities, and the angular size of the radio continuum emission at the $3 \sigma_{\mathrm{rms}}$ level. We also provide the name of the nearest maser from the sample and the angular separation between the continuum peak and the brightest spot of the nearest methanol maser.

The contour maps of all detections are shown in Figs. 2 and 3 . The majority of sources are single peaked and their angular sizes range from 0.' 6 to 3 .' $^{\prime}$. Both G24.148-00.009 and G36.115+00.552 have integrated flux densities that are equivalent to their peak flux densities within the noise, suggesting that these sources are unresolved. The values given for these sources in Table 7 correspond to the angular size upper limits. G31.582+00.075 is one of the weakest sources $\left(S_{\mathrm{p}}=\right.$ $0.43 \mathrm{mJy} \mathrm{beam}^{-1}$ ) but has an exceptionally complex structure. It is extended $\left(4^{\prime \prime} \times 3^{\prime \prime}\right)$ and contains multiple emission peaks. The typical upper limit $\left(3 \sigma_{\mathrm{rms}}\right)$ for the fluxes of the remaining 22 , non-detected sources is $0.15 \mathrm{mJy}$ beam $^{-1}$.

The $6.7-\mathrm{GHz}$ methanol maser emission is found to be within 0.2 of the $8.4-\mathrm{GHz}$ continuum position peaks of G24.148-00.009, G28.817+00.365, and G36.115+00.552. The maser spots of source G26.598-00.024 are 0. '8 from the NE edge of the radio continuum source. Therefore, these four sources are closely associated with the methanol masers (Fig. 2). The continuum object G31.160+00.045 is located $11^{\prime \prime}$.9 from the nominal position of the maser source G31.156+00.045, but this maser has a position uncertainty of $10^{\prime \prime}$ because we were unable to image it with the EVN (Sect. 3.1), so it may also be associated with the radio continuum. On the other hand, the continuum source G31.582+00.075 has a separation of 9 " from the maser $\mathrm{G} 31.581+00.077$, but the latter has a position accuracy of a few mas implying that the source and maser are unlikely to be associated.

We conclude that only 4 (possibly 5) of 30 masers are associated with radio continuum at $8.4 \mathrm{GHz}$. This is consistent with previous findings (Phillips et al. 1998; Walsh et al. 1998; Beuther et al. 2002) that the $6.7 \mathrm{GHz}$ methanol masers are rarely associated with centimeter wavelength continuum emission. However, 24-GHz ATCA observations detected continuum emission associated with methanol masers toward which no continuum at $8.4 \mathrm{GHz}$ had been previously detected (Longmore et al. 2007). This opens the possibility of methanol masers being associated with hyper-compact $\mathrm{H}$ II regions (HC H II), which are ptically thick at frequencies $<10 \mathrm{GHz}$.

\subsection{General properties of the $6.7 \mathrm{GHz}$ methanol masers}

In 31 sources, we detected a total of 1934 maser spots that form 333 clusters. The spectral profiles of 265 (80\%) clusters are well fitted with a Gaussian. The mean FWHM is $0.41 \pm$ $0.01 \mathrm{~km} \mathrm{~s}^{-1}$ and the median value is $0.37 \mathrm{~km} \mathrm{~s}^{-1}$. This is consistent with results from single dish spectra at $\sim 0.05 \mathrm{~km} \mathrm{~s}^{-1}$ resolution (Menten 1991; Caswell et al. 1995). Nineteen sources have complex spectra, that is indicative of spectral blending, so that the line width of individual features cannot be properly determined solely from the spectrum.

We compared the basic parameters of the spectra and distributions of all masers from the sample. However, we did not find any relationships between the line parameters such as $F W H M$, brightness temperature, velocity range of the maser emission, and the size and geometry of the maser region.

The sources show a wide diversity of structures. The following types of morphology can be identified (Table 5):

Simple - the emission appears in a narrow velocity range $\left(\Delta V=0.7 \mathrm{~km} \mathrm{~s}^{-1}\right)$ as a single peaked spectrum. The maser spots form one cluster of size smaller than a few mas. G37.030-00.039 is the only source with these properties. Its spectrum is obviously blended.

Linear - the maser spots form a line in the plane of the sky. The angular extent of these maser structures ranges from 9 to 54 mas. In some sources (G30.318+00.070, G35.793-00.175) a monotonic velocity gradient is clearly seen. There are five linear sources in the sample.

Ring - this morphology appears to be ubiquitous in our sample. The distributions of no less than nine sources display a ring structure. Using the GNU Octave script developed by Fitzgibbon et al. (1999), we fitted an ellipse to the spatial positions of the maser spots for each source. The results are summarized in Table 8. The semi-major (a) and semi-minor (b) axes range from 27 to 192 mas and from 15 to 128 mas, respectively. The average size of the semi-major axis and the standard dispersion in the mean is $89 \pm 20$ mas. The eccentricity (e) of the best-fit model ellipses ranges from 0.38 to 0.94 . The average eccentricity and the standard dispersion of the mean is $0.79 \pm 0.06$. The emission spans a modest velocity range of $\left(3.1-13.4 \mathrm{~km} \mathrm{~s}^{-1}\right)$. All nine sources possess MIR counterparts that coincide with the ellipse center to within less than 2 '. 5 (Table 5). In these objects it is very likely that ring-like maser emission surrounds a central embedded star (see Sect. 4.2). Three other sources $(G 28.817+00.365$, G30.400-00.296, G31.581+00.077) have a ring-like morphology, although the separation between the MIR candidate counterpart and the ellipse center is greater than $2 . .5$ (Table 5). This is probably caused by the larger uncertainties in the maser 

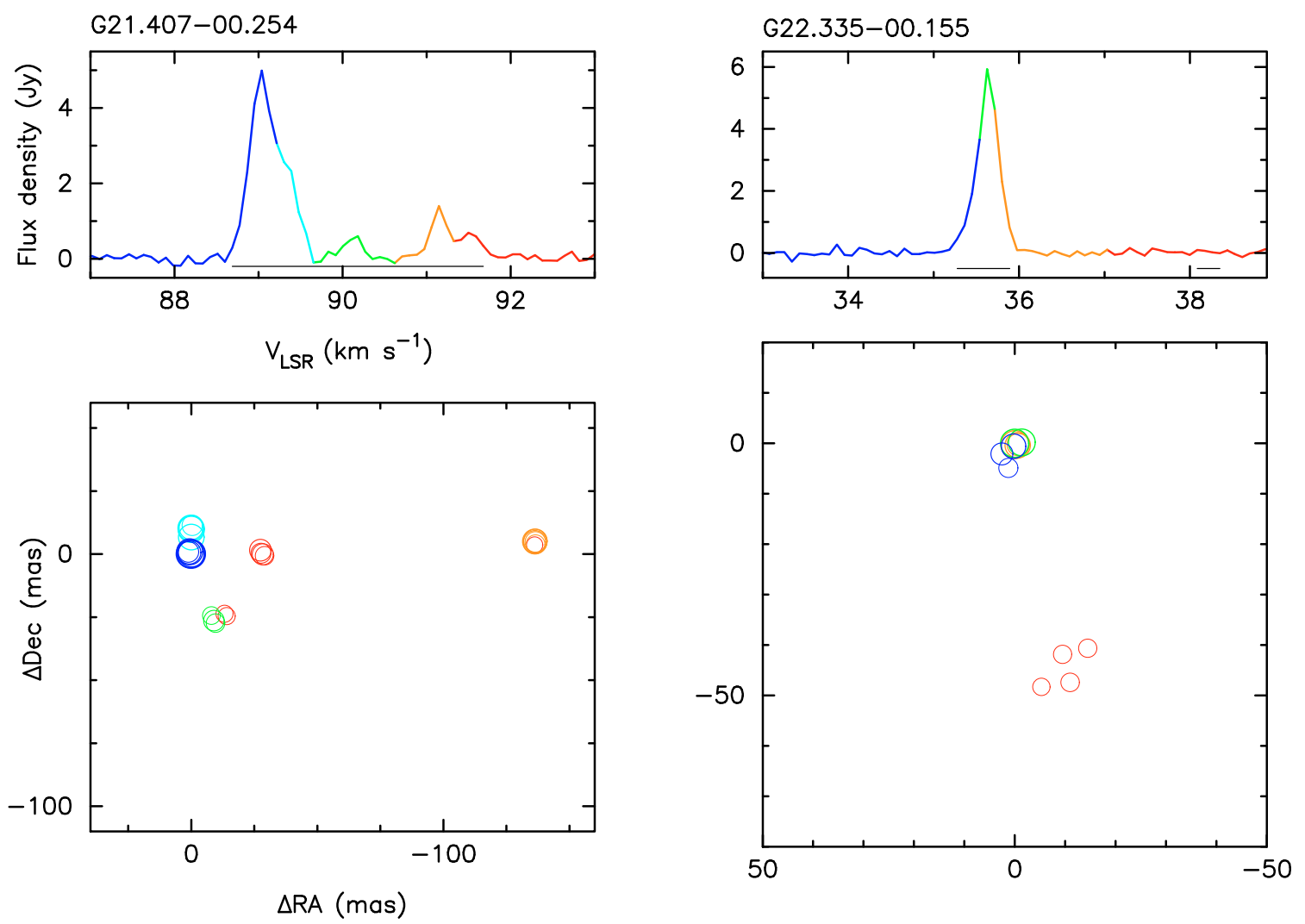

$\mathrm{G} 22.357+00.066$

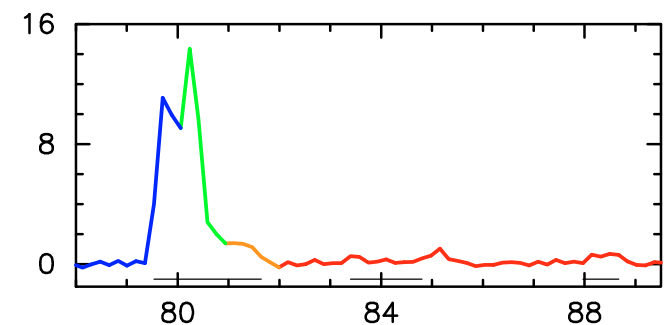

\section{G23.207-00.377}
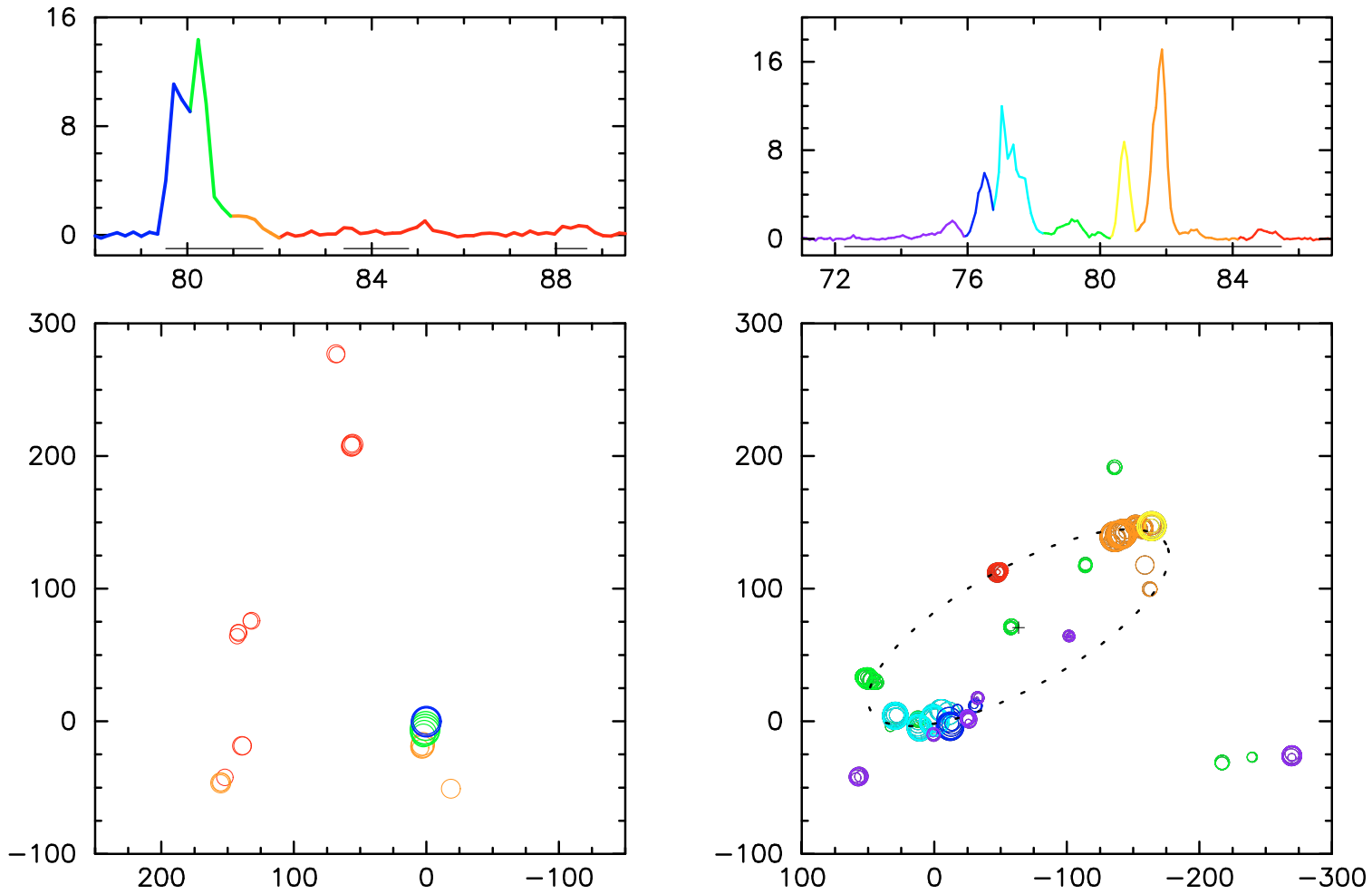

Fig. 1. Spectra and maps of $6.7 \mathrm{GHz}$ methanol maser emission of sources detected with the EVN. The names are the Galactic coordinates of the brightest spot as listed in Table 5. The thin bars under the spectra show the velocity ranges of spots displayed. The coordinates are relative to the brightest spots (Table 5). The sizes of circles are proportional to the logarithm of the intensities of maser spots. The colors of circles relate to the LSR velocities as indicated in the spectra, respectively. For the sources with ring-like morphologies, the best-fit ellipse and its center are marked by a dotted curve and a cross, respectively. The crosses coincide (within the uncertainties) with Spitzer IRAC MIR emission (Sect. 4.2). 
G23.389+00.185
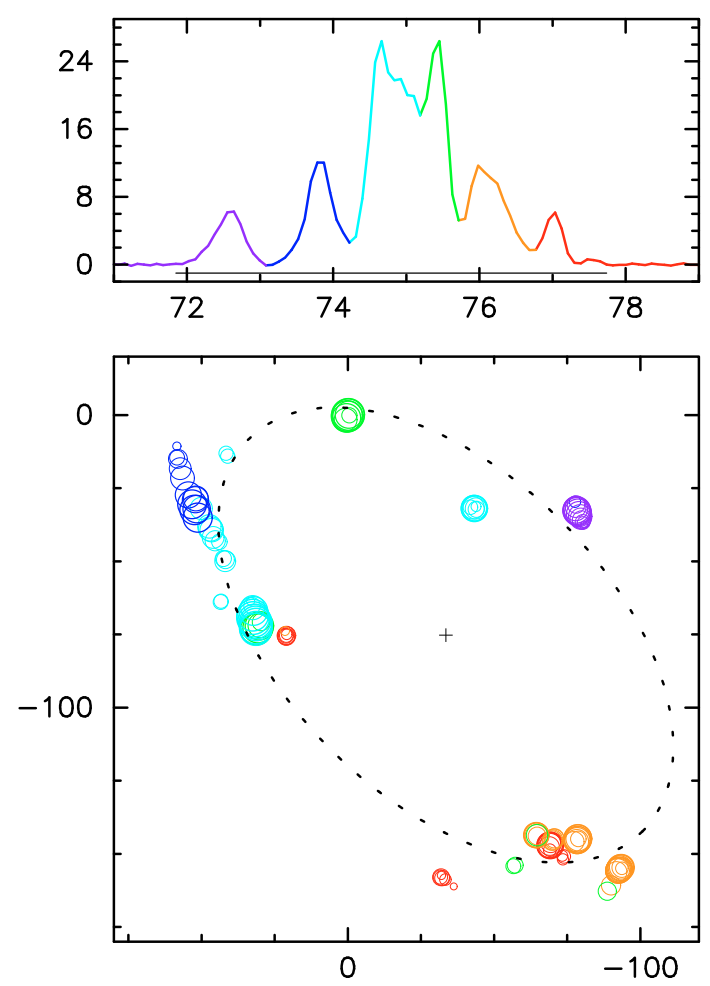

G23.707-00.198
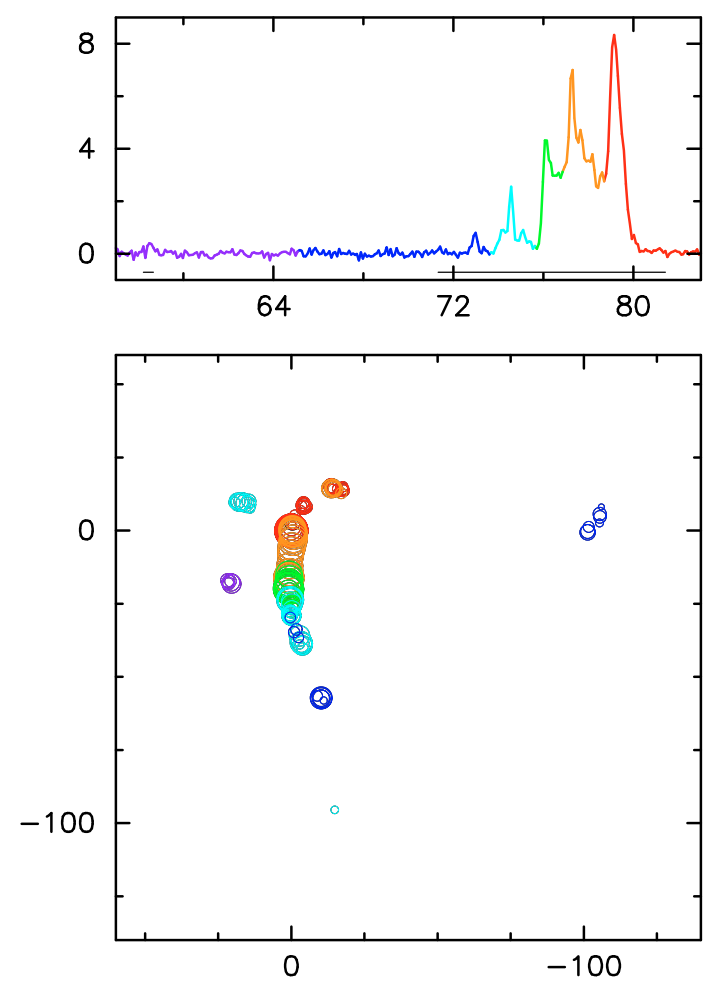

G23.657-00.127
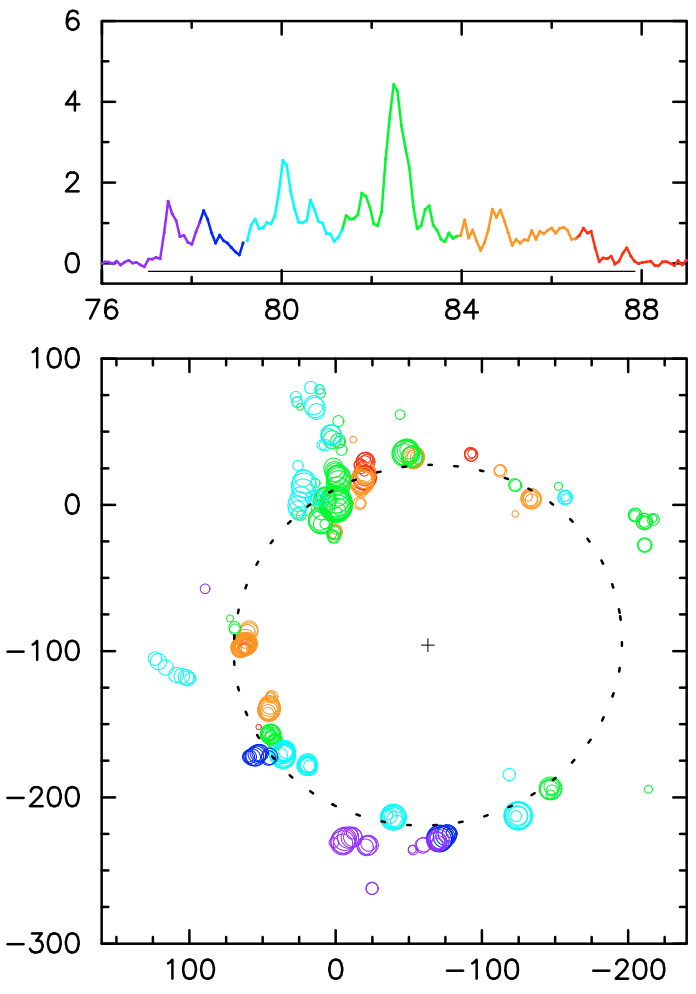

G23.966-00.109
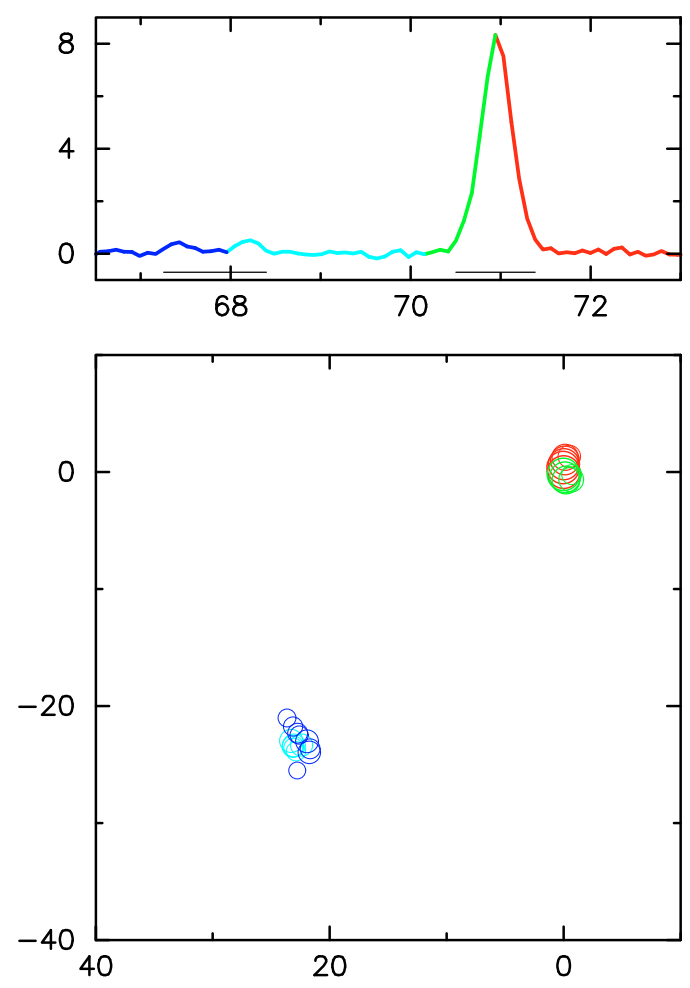

Fig. 1. continued.

positions, since all three sources are at declinations near $0^{\circ}$. These are assigned a tentative classification of the ring-like class in Table 5.
Arched - maser spots are distributed along an arc of between 70 and 220 mas in length. The entire structure may show a systematic velocity gradient. Three (or possibly five) sources exhibit this morphology. 
G24.148-00.009
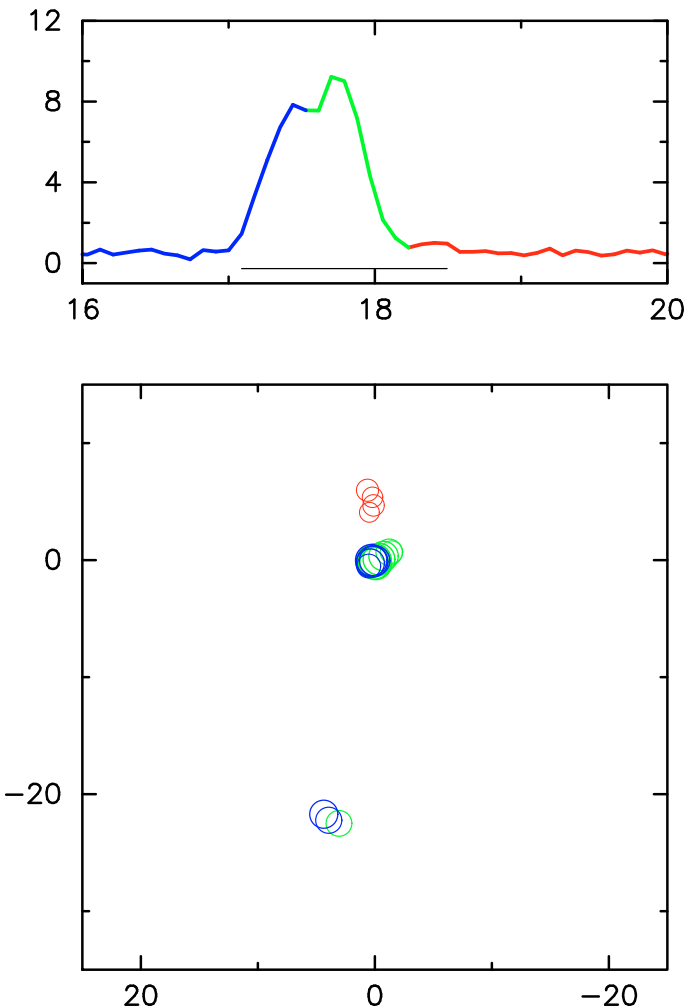

G24.634-00.324
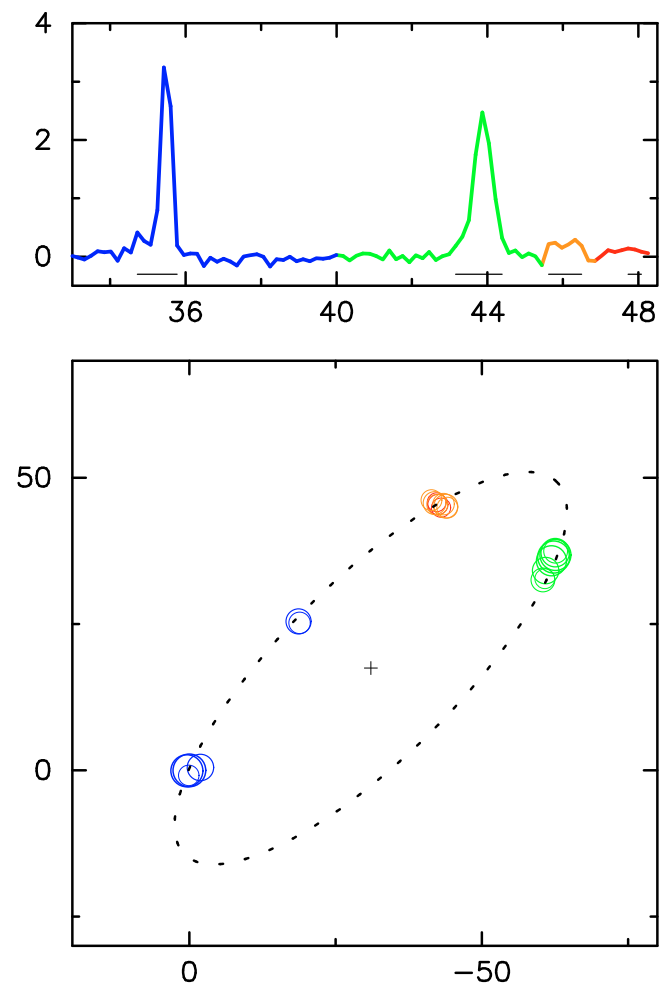

$\mathrm{G} 24.541+00.312$
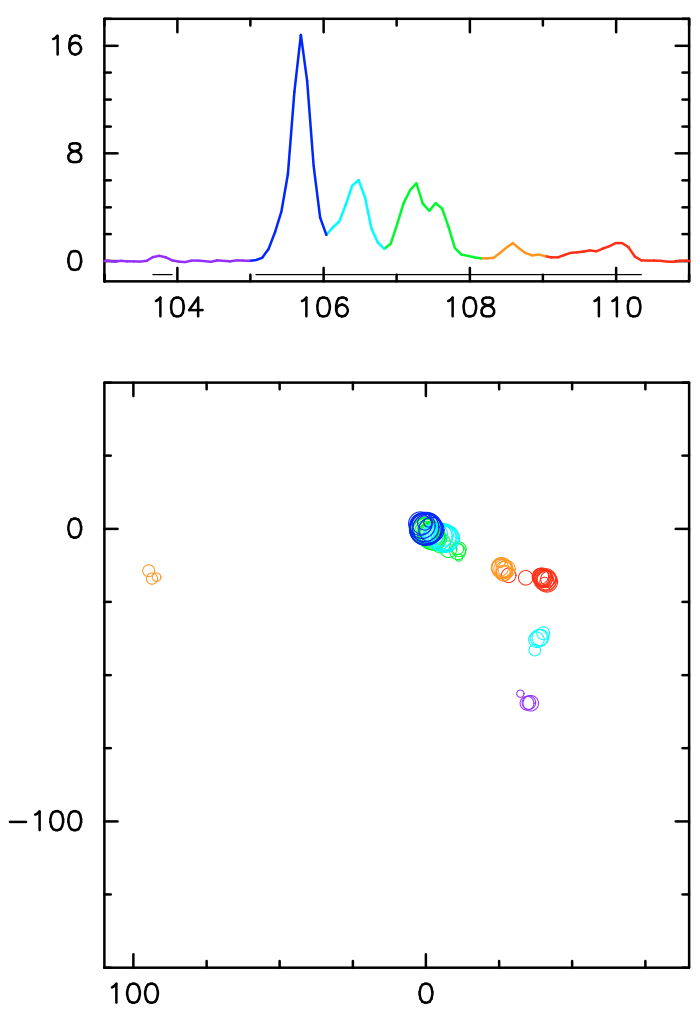

$\mathrm{G} 25.411+00.105$
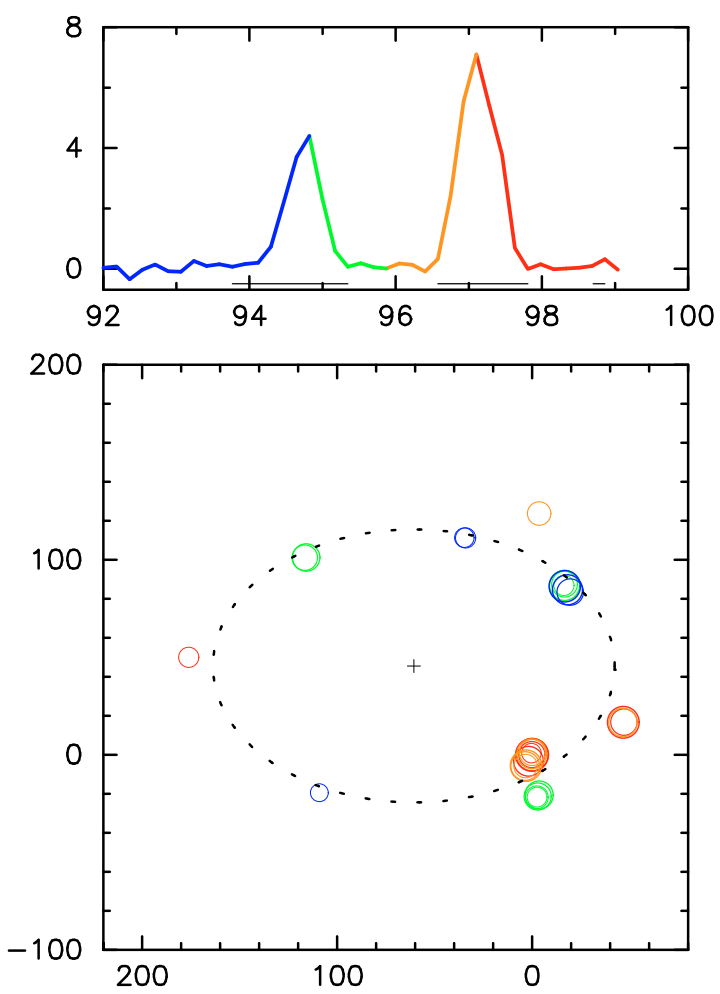

Fig. 1. continued.

Complex - 9 (possibly 10) sources, i.e. about one third of the sample, do not show any regularities in their spatial and velocity distributions. These sources vary greatly in size from $31 \times 23$ mas $^{2}$ to $330 \times 174$ mas $^{2}$.
Pair - this class was defined by Phillips et al. (1998), comprising two maser groups separated by $\sim 1$ arcsec with $\geq 10 \mathrm{~km} \mathrm{~s}^{-1}$ difference in velocity. The major axes of individual clusters are perpendicular to the line joining them. In our sample, we found only one source with such a morphology. 


\section{G26.598-00.024}
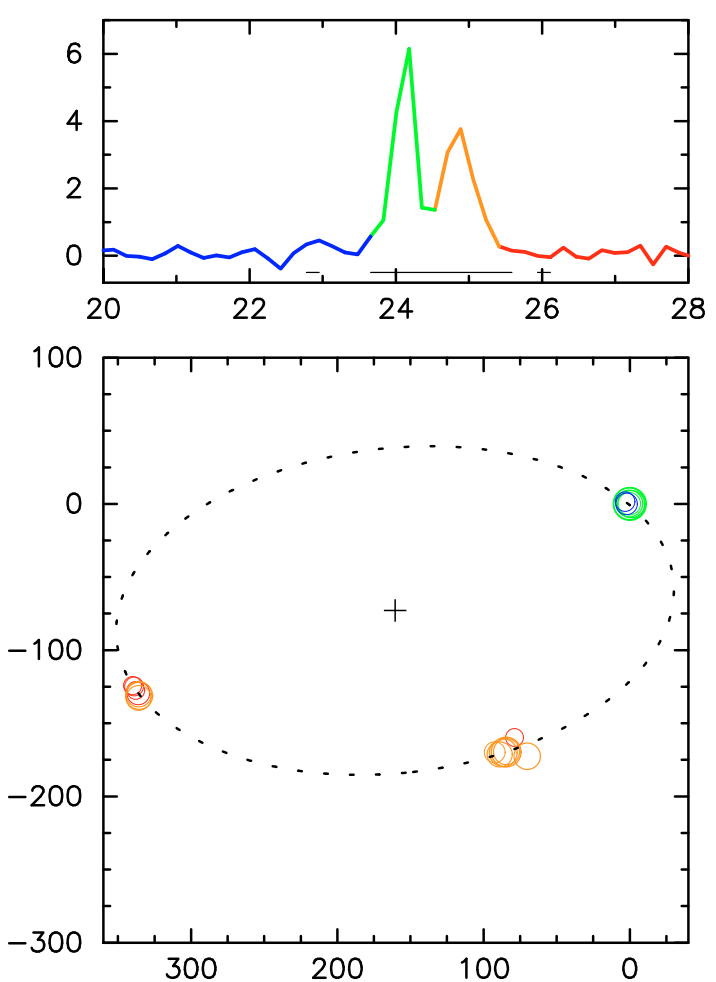

$\mathrm{G} 28.817+00.365$
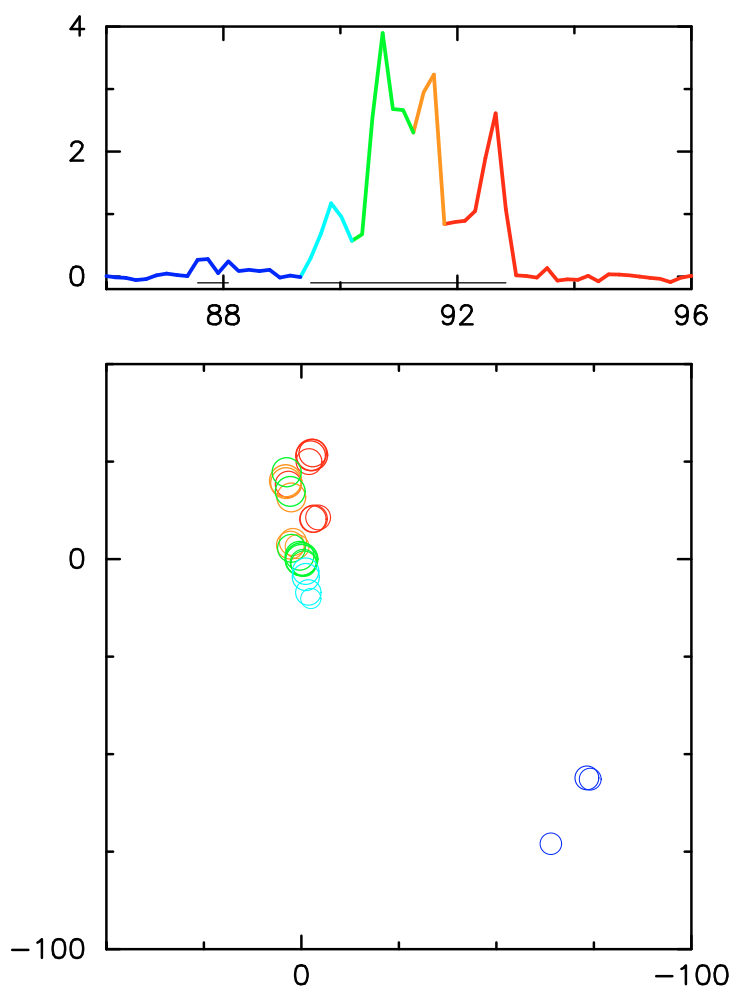

G27.221+00.136
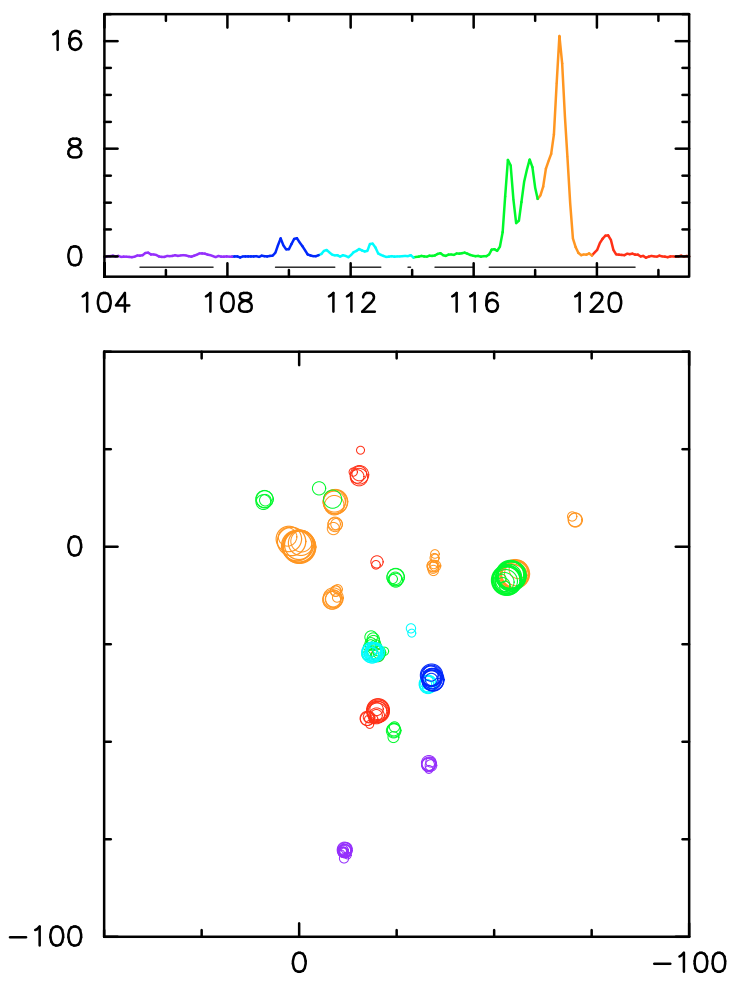

G30.318+00.070
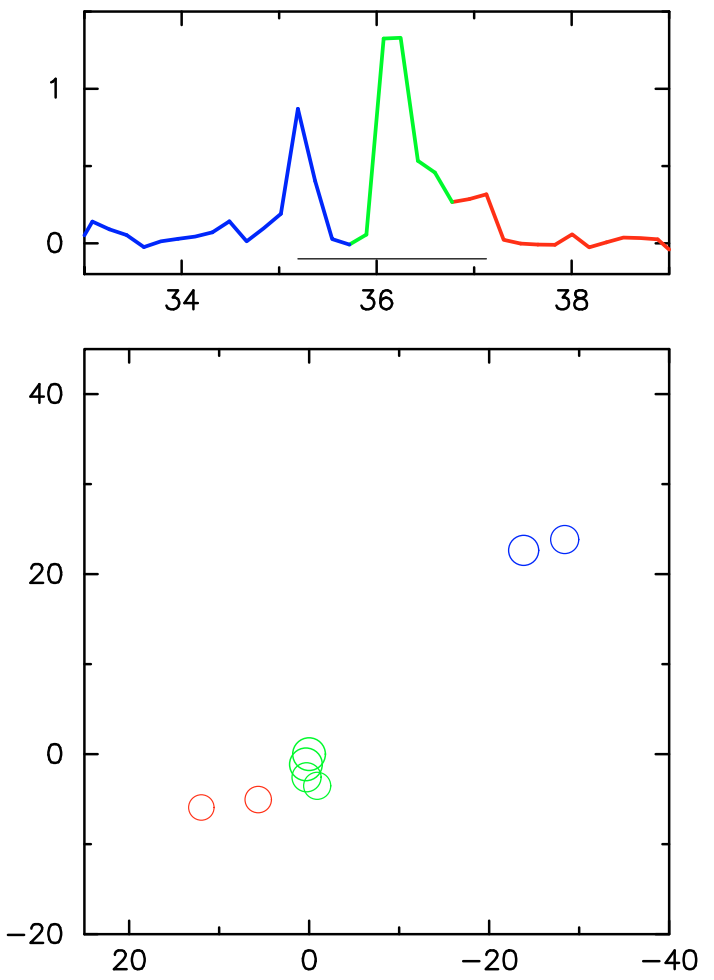

Fig. 1. continued.

The most striking aspect of this study is that we find that the commonest morphology of sources with a systematic maser structure is a ring-like distribution of emission, seen in $~ 29 \%$ of objects. These rings probably surround young massive objects (see Sect. 4). A similar proportion ( 29-32\%) of sources possess a complex morphology. Linear sources with a monotonic velocity gradient are relatively rare in the sample $(\sim 16 \%)$. 
G30.400-00.296
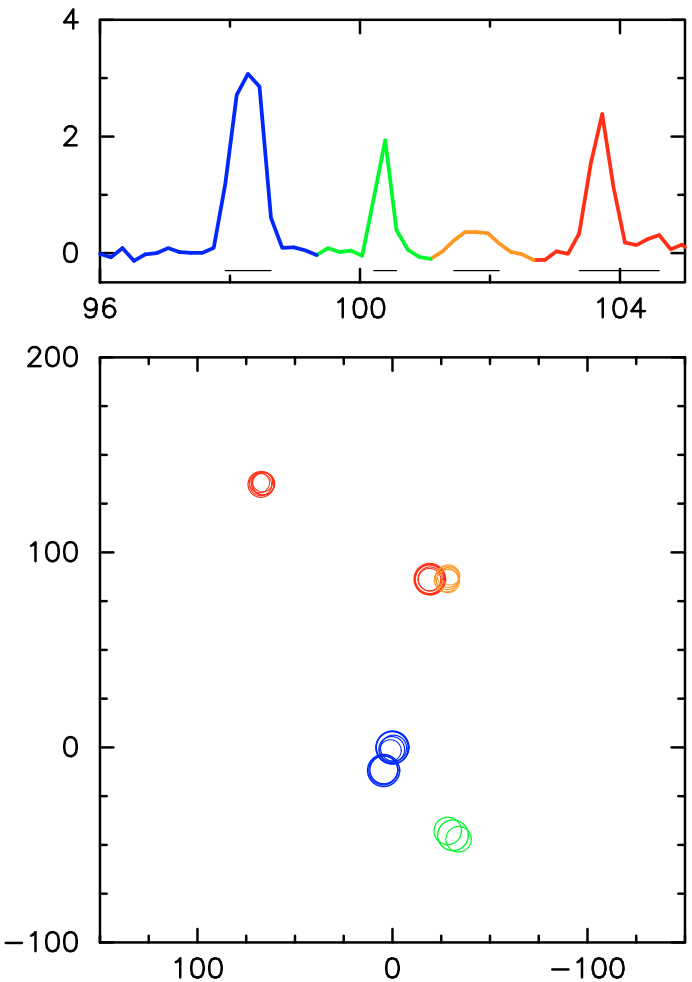

G31.581+00.077
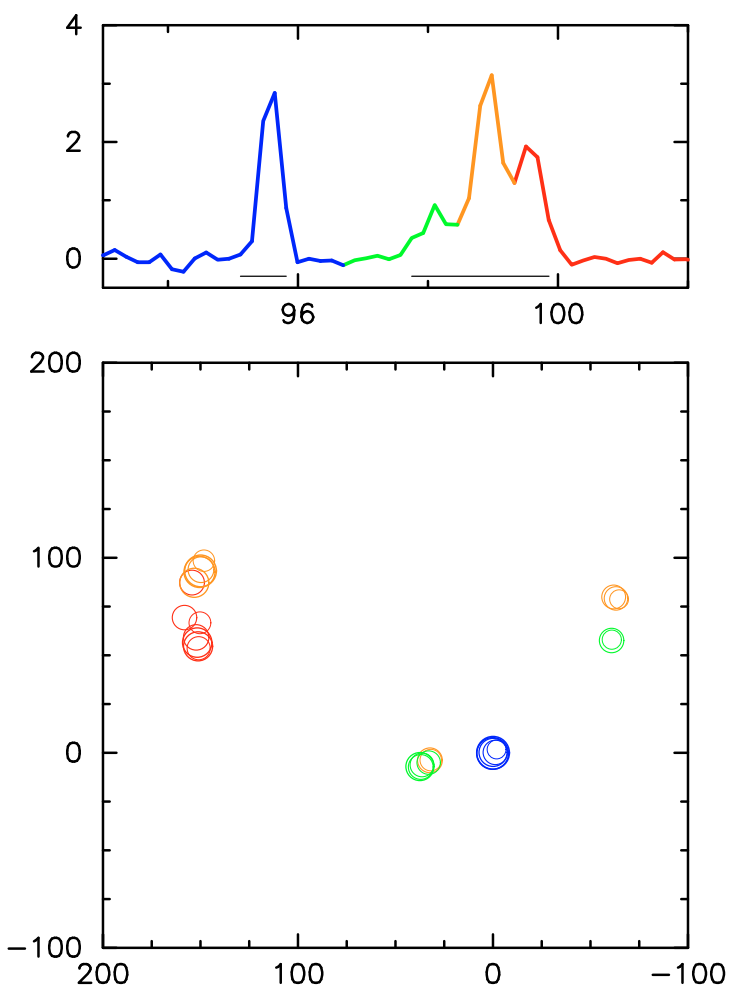

Fig. 1. continued.

\subsection{Individual sources}

This section presents comments on individual sources, including additional observational data relevant to the main aims of this paper. If not stated otherwise, we present the linear sizes
G31.047+00.356
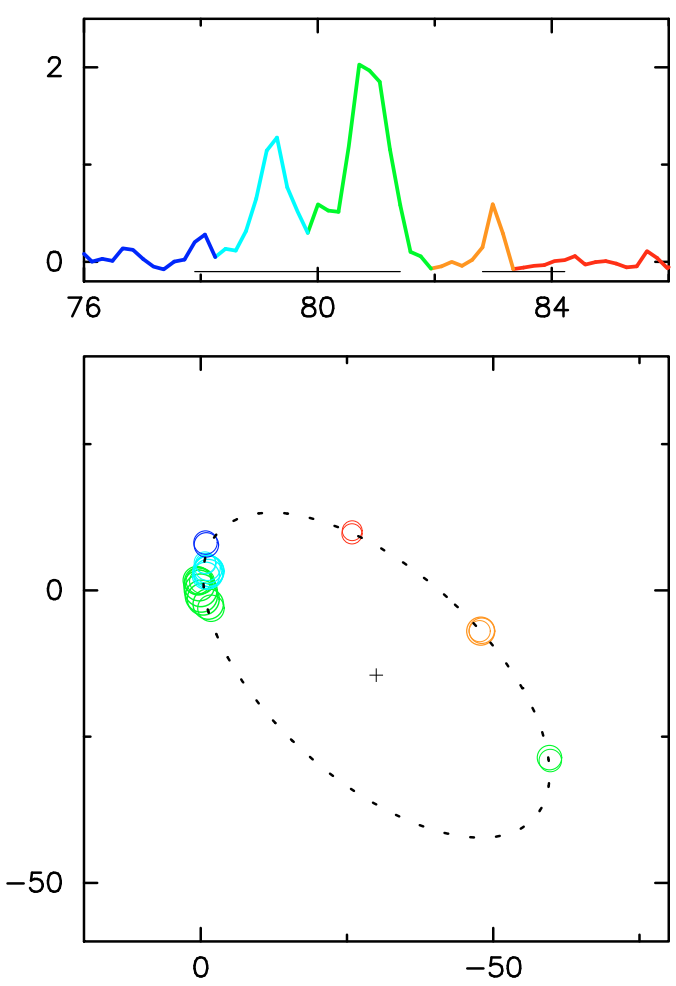

$\mathrm{G} 32.992+00.034$
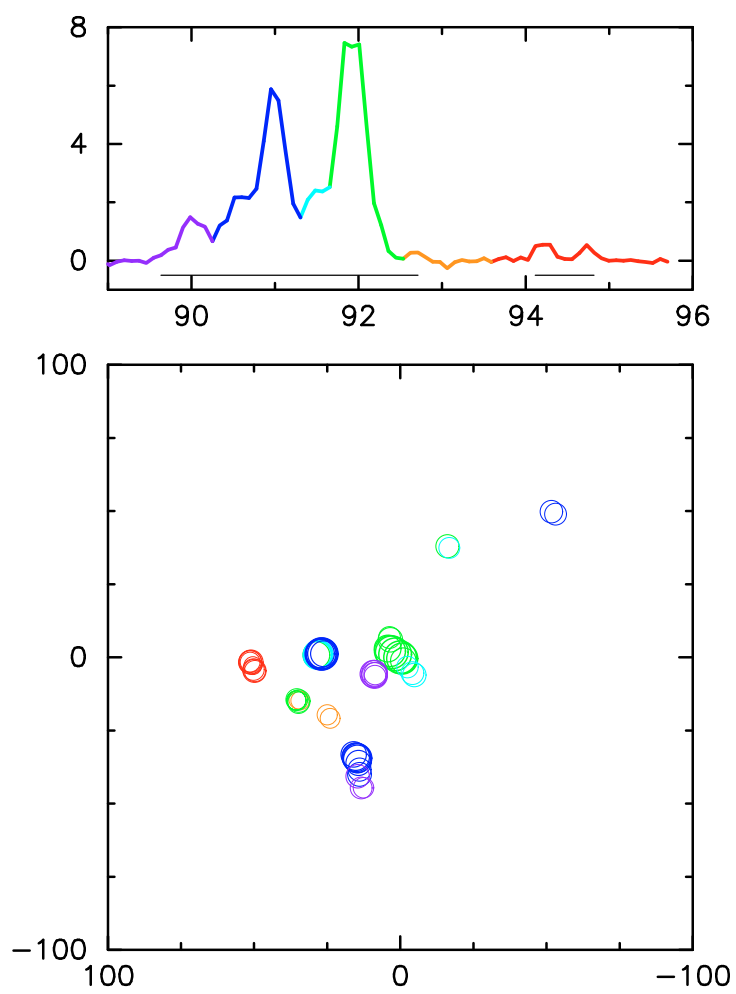

of masers derived using the near (and far) kinematic distances given in Szymczak et al. (2005).

G22.357+00.066. The ATCA observations detected three maser spots (Walsh et al. 1998), while the EVN revealed 31 spots in 10 clusters. The strongest emission detected with both 

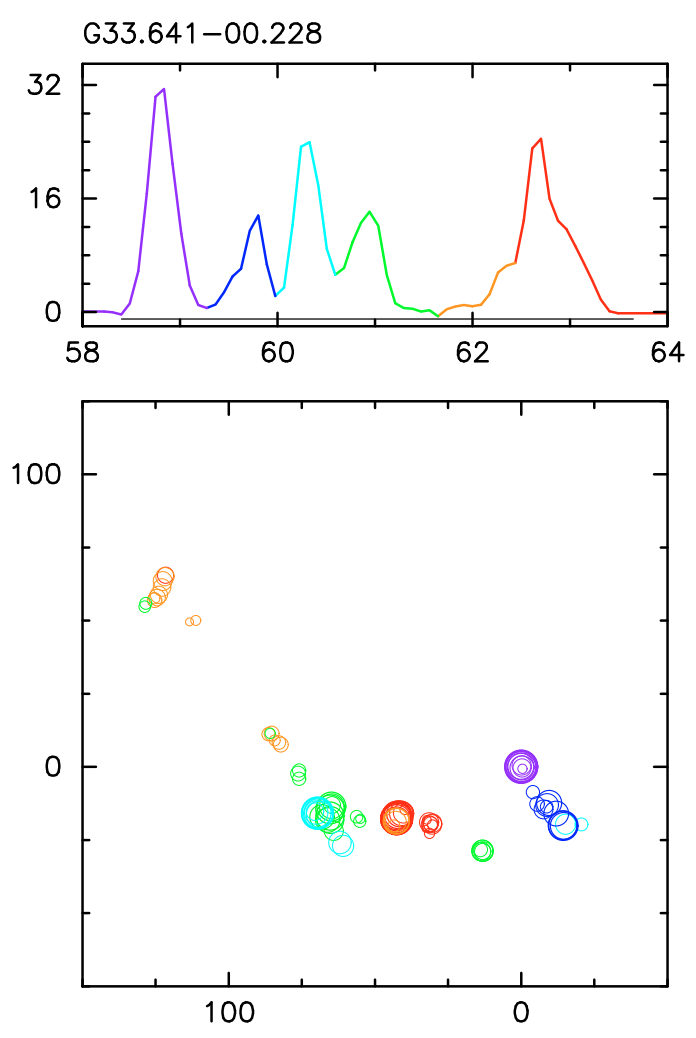

\section{G34.751-00.093}
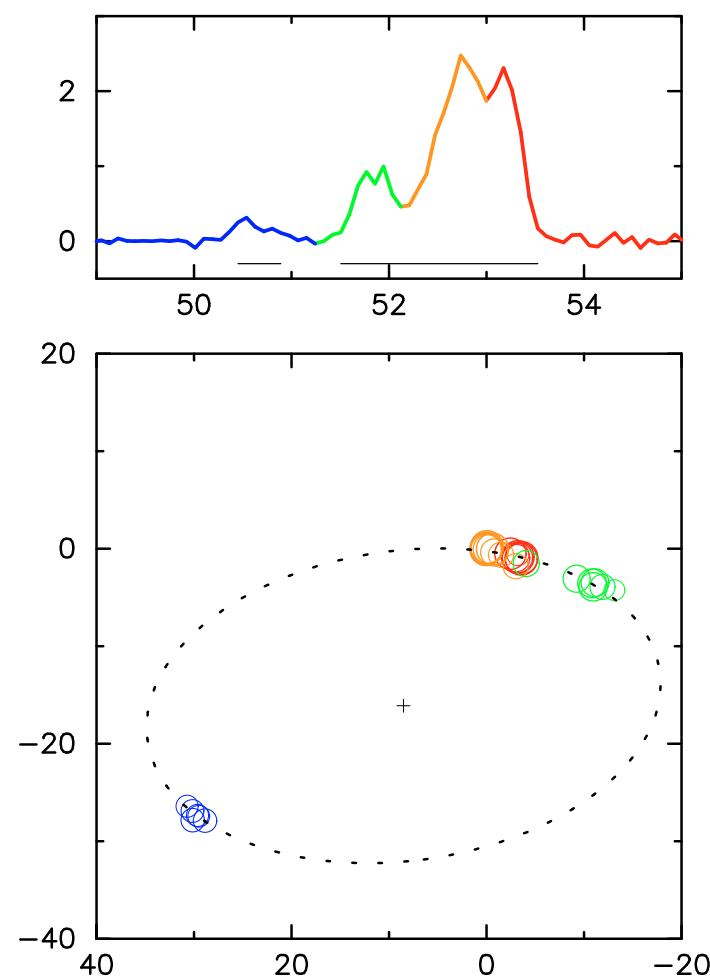

Fig. 1. continued.

interferometers, at $80.0 \mathrm{~km} \mathrm{~s}^{-1}$, coincides to within 1 .'7. We detected new emission at close to $88.5 \mathrm{~km} \mathrm{~s}^{-1}, 0.20-0.27$ north of the brightest spot, but a $77.0 \mathrm{~km} \mathrm{~s}^{-1}$ spot reported by Walsh et al. (1998) was not redetected.
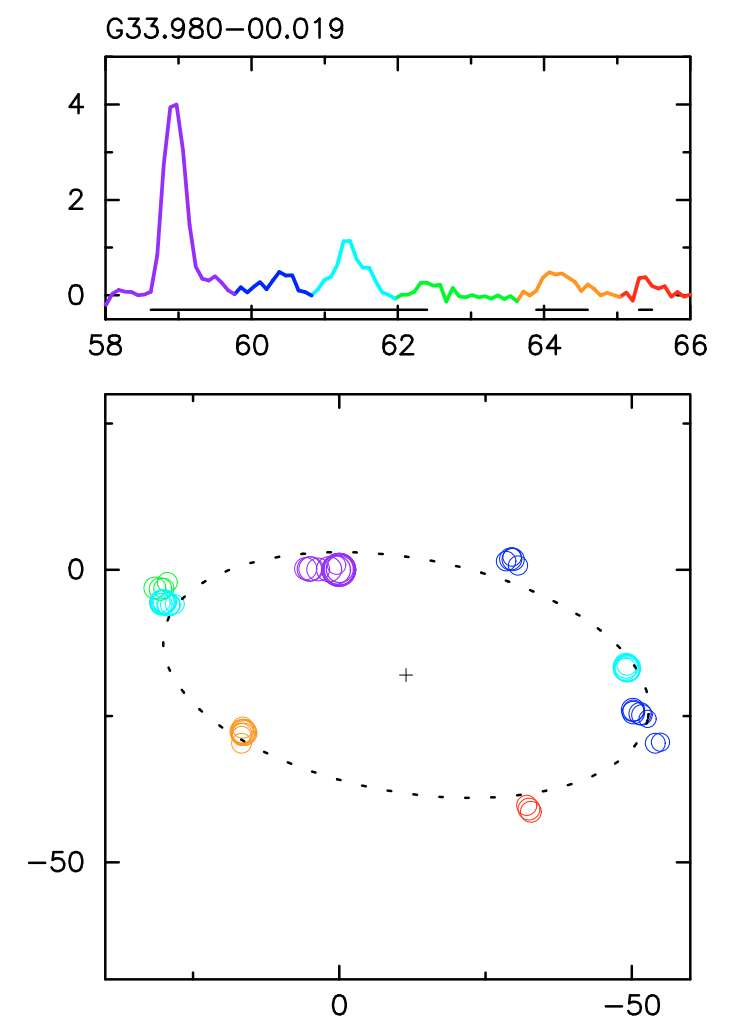

G35.793-00.175
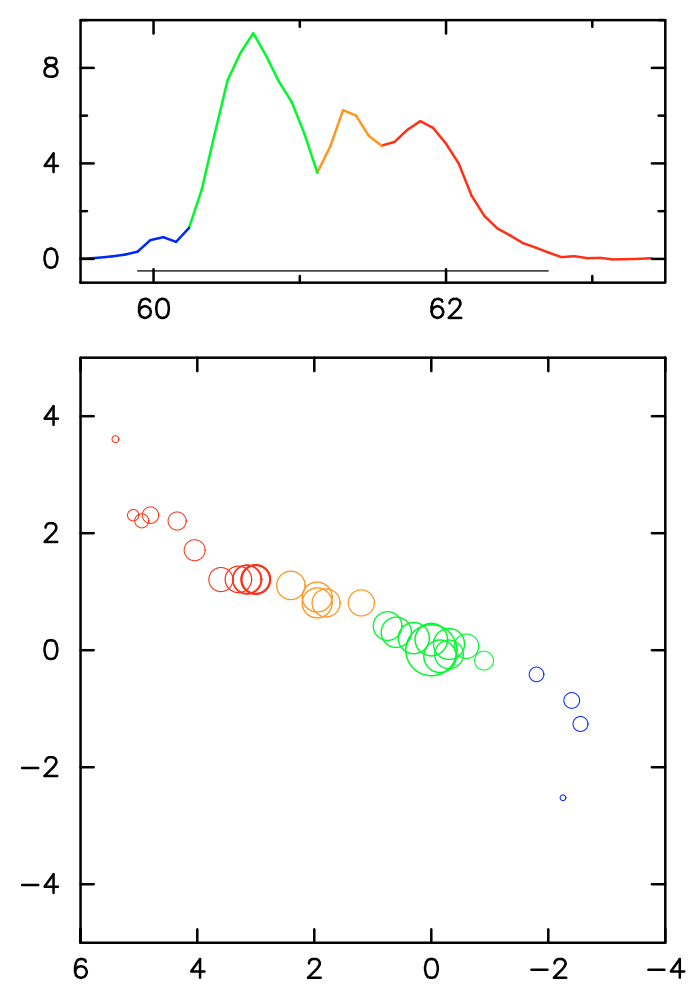

G23.657-00.127. The parallax of this source was measured by Bartkiewicz et al. (2008) and its value indicates that the circular distribution of masers has a linear radius of $405 \mathrm{AU}$, which differs significantly from the sizes inferred from the kinematic distances. The source was observed at three epochs (Tables 2, 4) 

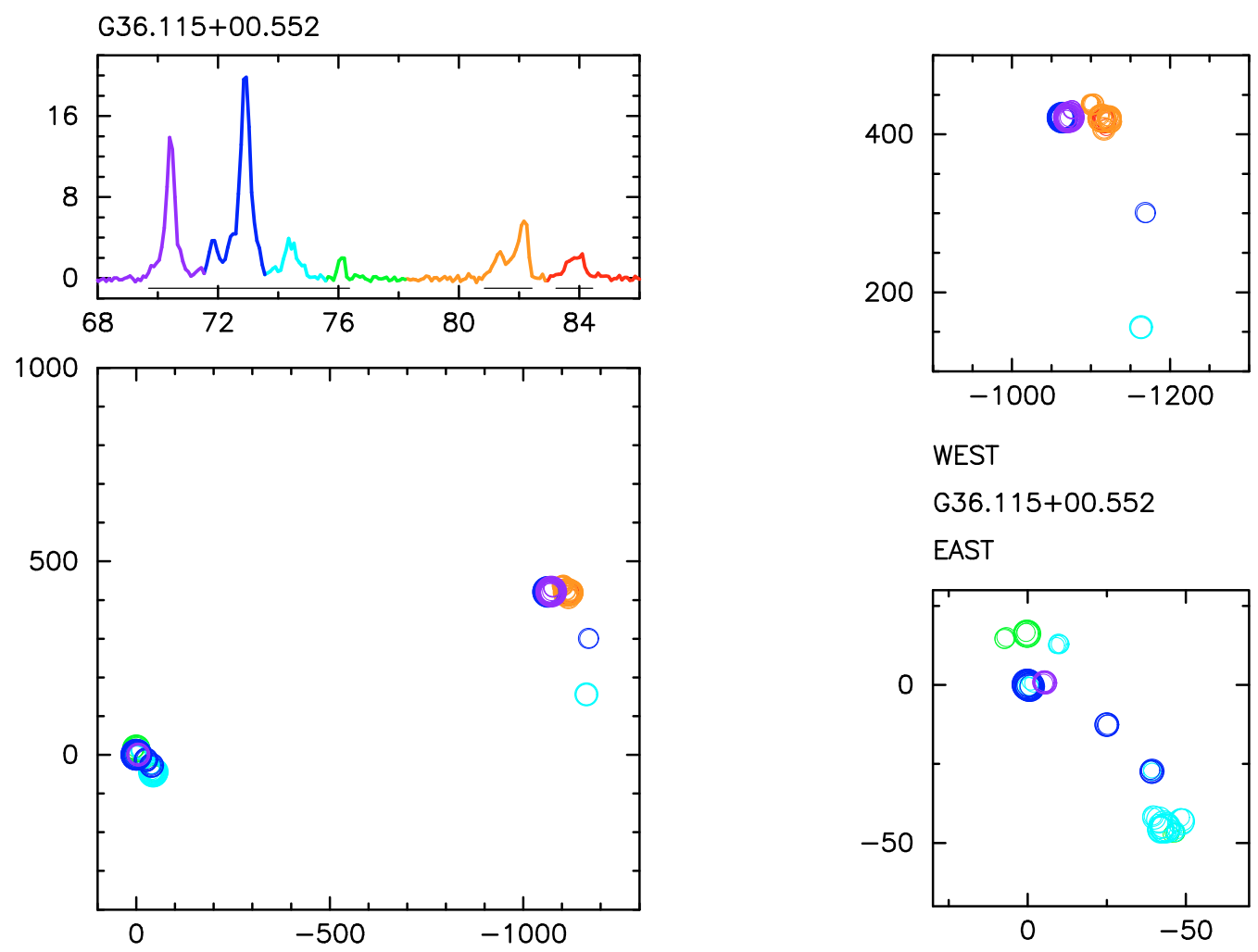

WEST

G36.115+00.552

EAST

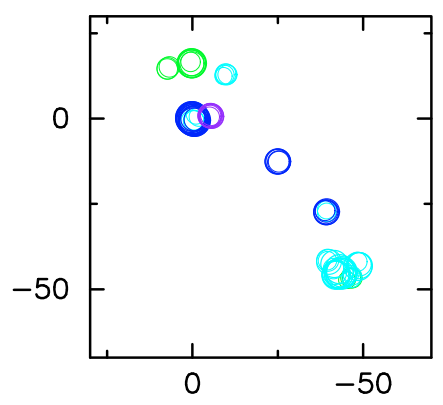

G36.705+00.096

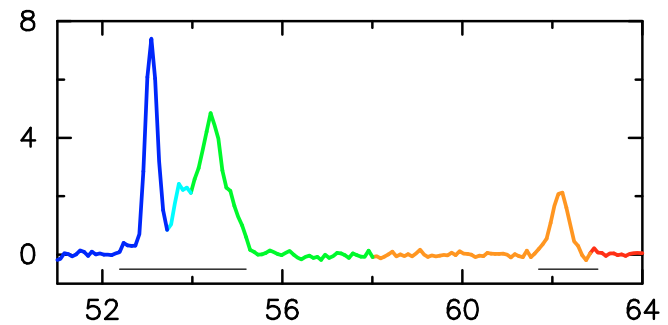

G37.030-00.039
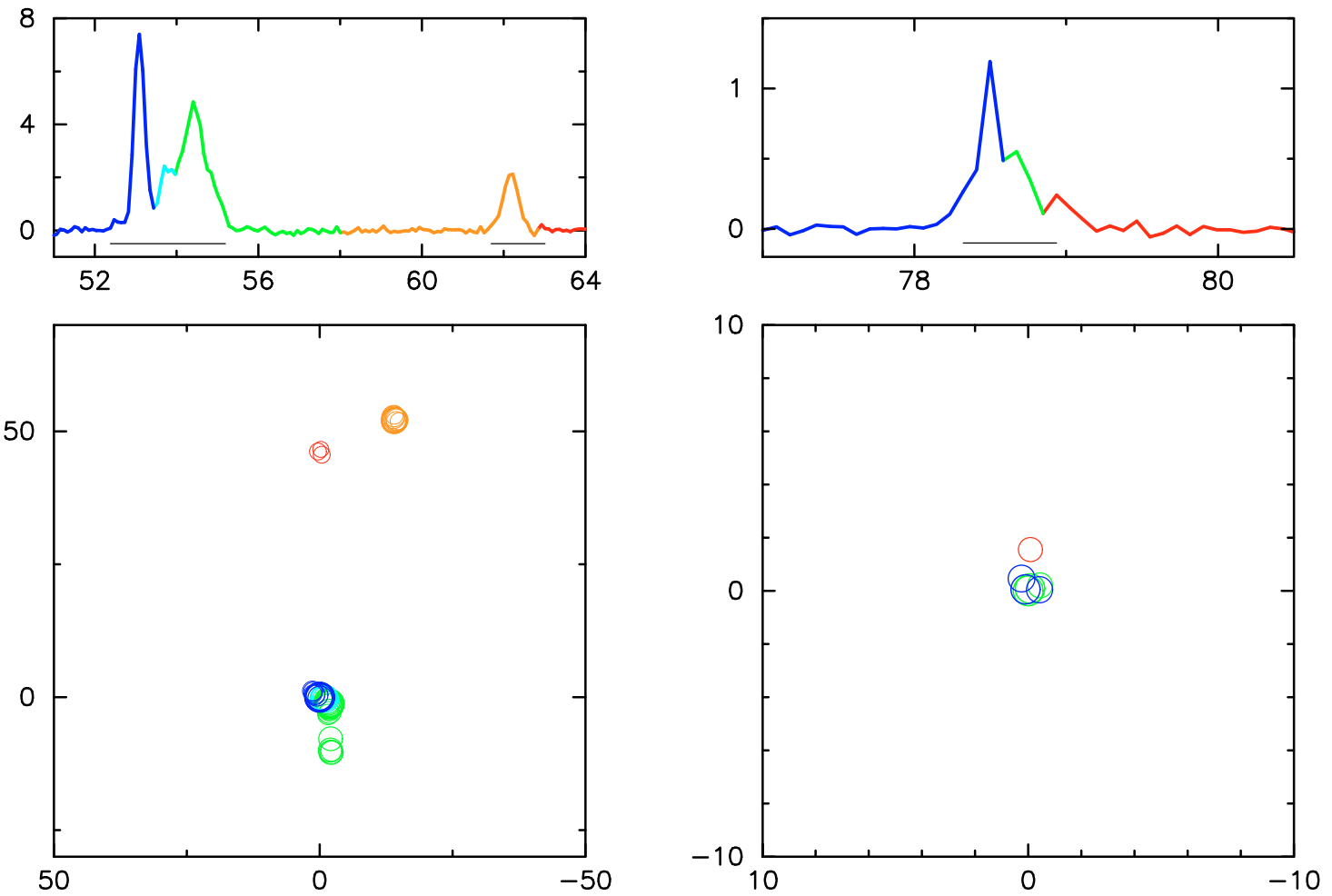

Fig. 1. continued.

and the ring-like morphology clearly persisted over time spans of 1.25 and $2.5 \mathrm{yr}$. A detailed description of the brightness variability in the source will be presented in a forthcoming paper.

G23.707-00.198. Walsh et al. (1998) detected 7 masers in a velocity range of $74.9-81.4 \mathrm{~km} \mathrm{~s}^{-1}$, randomly scattered over

a 0.15 area. The first epoch of EVN maps of this source (run 2) detected 23 clusters (140 spots) of which 19 form a 71 mas (corresponding to 360/750 AU for near/far kinematic distance, respectively) long arc in the NS direction, which has a velocity span of $8 \mathrm{~km} \mathrm{~s}^{-1}$. A clear velocity gradient is seen in the overall 
G37.598+00.425
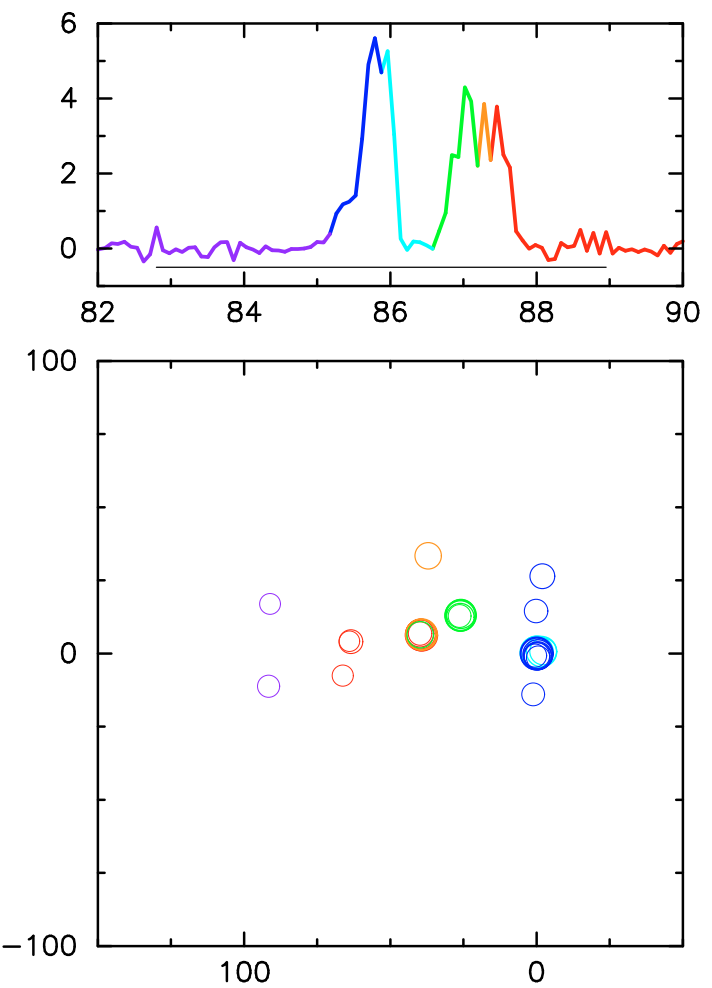

G38.203-00.067
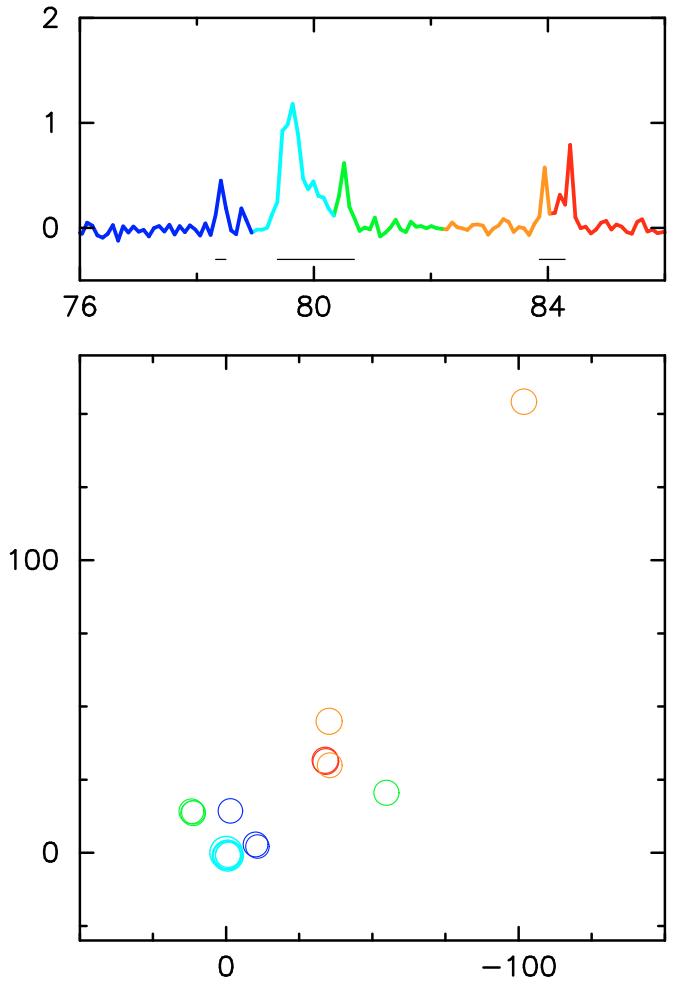

Fig. 1. continued.

arched distribution. The remaining clusters (all blue-shifted) are located $\sim 100$ mas to the west (two clusters) and $\sim 20$ mas to the east (two clusters), relative to the brightest spot. All four clusters are weak and were not detected at a later epoch (run 3a), but this data had poorer sensitivity. The brightest methanol maser
G38.038-00.300
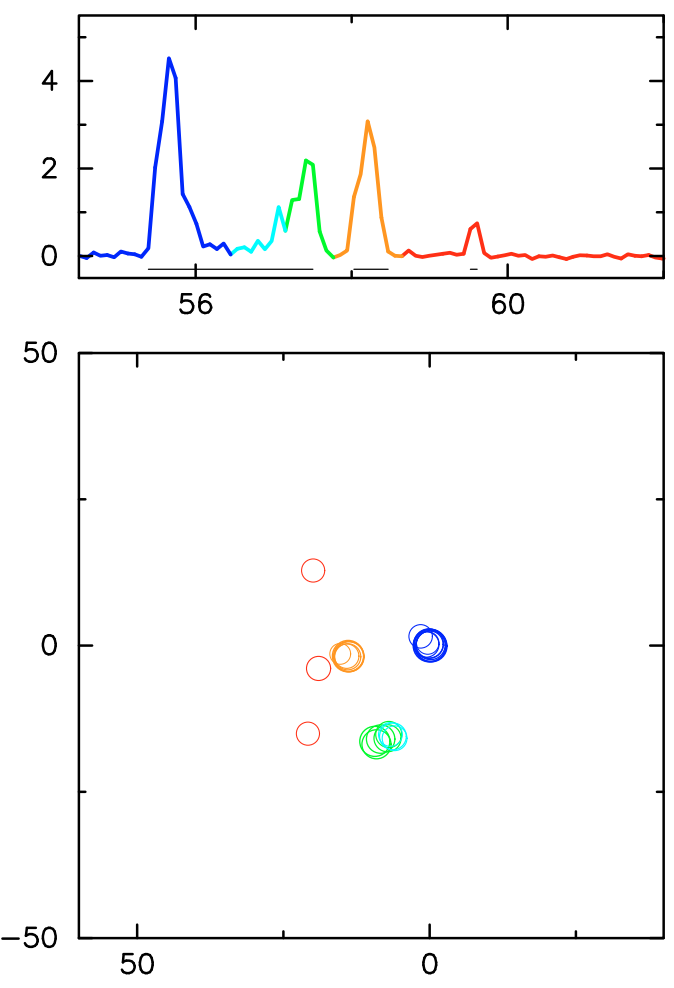

G39.100+00.491
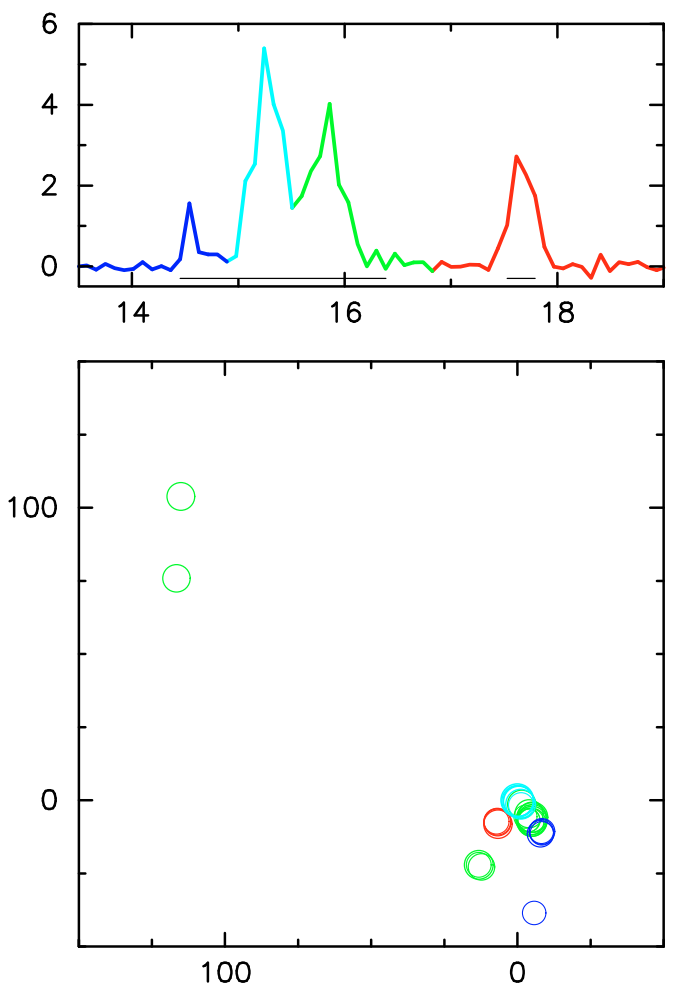

component (Table 5) coincides in position (within 82 mas) and velocity (within $0.1 \mathrm{~km} \mathrm{~s}^{-1}$ ) with weak $\left(60 \mathrm{mJy} \mathrm{beam}^{-1}\right) \mathrm{H}_{2} \mathrm{CO}$ maser emission at $4.8 \mathrm{GHz}$ (Araya et al. 2006). We note that this is well within the absolute positional accuracy of the $\mathrm{H}_{2} \mathrm{CO}$ maser. Both masers lie at the edges of two probable $\mathrm{H}$ II regions 
Table 7. Results of VLA observations at $8.4 \mathrm{GHz}$.

\begin{tabular}{lccccccc}
\hline \hline $\begin{array}{l}\text { Radio continuum } \\
\text { source }\end{array}$ & $S_{\mathrm{p}}$ & $\begin{array}{c}S_{\text {int }} \\
\left(\mathrm{mJy}_{\text {beam }}^{-1}\right)\end{array}$ & $\begin{array}{c}\text { Major axis } \\
\left({ }^{\prime \prime}\right)\end{array}$ & $\begin{array}{c}\text { Size } \\
\text { Minor axis } \\
\left({ }^{\prime \prime}\right)\end{array}$ & $\begin{array}{c}\text { PA } \\
\left({ }^{\circ}\right)\end{array}$ & Nearest maser & Separation \\
\hline G21.385-00.254 & 13.00 & 65 & 3.8 & 1.8 & +50 & G21.407-00.254 & 76.8 \\
G24.148-00.009 & 1.05 & 1 & $0.6^{*}$ & $0.4^{*}$ & +35 & G24.148-00.009 & 0.11 \\
G26.598-00.024 & 4.30 & 42 & 3.8 & 2.5 & -35 & G26.598-00.024 & 0.80 \\
G28.817+00.365 & 0.81 & 0.8 & 0.6 & 0.5 & -20 & G28.817+00.365 & 0.08 \\
G30.330+00.090 & 8.70 & 13 & 1.3 & 0.8 & -25 & G30.318+00.070 & 85.6 \\
G31.160+00.045 & 2.40 & 22 & 2.0 & 1.5 & +70 & G31.156+00.045 & 11.9 \\
G31.582+00.075 & 0.43 & 15 & 4.0 & 3.0 & 0 & G31.581+00.077 & 9.00 \\
G36.115+00.552 & 0.25 & 0.2 & $0.7^{*}$ & $0.3^{*}$ & -45 & G36.115+00.552 & 0.20 \\
\hline
\end{tabular}

* Angular size upper limits (see Sect. 3.2).

Table 8. Parameters of ellipses fitted to the maser spot distributions.

\begin{tabular}{lccccc}
\hline \hline Source & $\begin{array}{c}\text { Centre } \\
\Delta \text { RA, } \Delta \text { Dec.* }\end{array}$ & \multicolumn{2}{c}{ Semi-axes } & PA $^{* *}$ & $e$ \\
& (mas, mas) & (mas) & (mas) & $\left(^{\circ}\right)$ & \\
\hline G23.207-00.377 & $-62,71$ & 126 & 45 & -60 & 0.93 \\
G23.389+00.185 & $-34,-75$ & 95 & 56 & +45 & 0.81 \\
G23.657-00.127 & $-69,-93$ & 133 & 128 & -10 & 0.38 \\
G24.634-00.324 & $-31,17$ & 45 & 15 & -45 & 0.94 \\
G25.411+00.105 & 61,46 & 103 & 70 & +90 & 0.73 \\
G26.598-00.024 & $161,-73$ & 192 & 111 & -84 & 0.81 \\
G31.047+00.356 & $-30,-15$ & 37 & 18 & +47 & 0.87 \\
G33.980-00.019 & $-11,-18$ & 42 & 20 & +80 & 0.88 \\
G34.751-00.093 & $9,-16$ & 27 & 16 & -83 & 0.80 \\
\hline
\end{tabular}

* Coordinates relative to the brightest spots as listed in Table 5. ${ }^{* *}$ The position angle of semi-major axis (north to east).

(Araya et al.2006, their Fig. 2) with a peak intensity of $6.1 \mathrm{mJy}^{-1}$ beam ${ }^{-1}$ at $5 \mathrm{GHz}$ (VLA C-configuration). Our VLA Aconfiguration data at $8.4 \mathrm{GHz}$ do not show any emission above the $0.15 \mathrm{mJy}_{\text {beam }}{ }^{-1}$ sensitivity limit, nor was this source detected at $8.6 \mathrm{GHz}$ with a $2 \mathrm{mJy}_{\text {beam }}{ }^{-1}$ limit (Walsh et al. (1998). Therefore, the $\mathrm{H}$ II regions are intrinsically weak at frequencies $>5 \mathrm{GHz}$ or they are possibly resolved at subarcsec angular resolution.

G25.411+00.105. Beuther et al. (2002) observed this source with the ATCA and found only two components at velocities of 94 and $97 \mathrm{~km} \mathrm{~s}^{-1}$ at positions that coincide to within 0.2 with the brightest spots in the EVN maps. We detected 30 spots, probably because of our $\sim 50$ times higher sensitivity, although variability may also be involved. The distance of $9.5 \mathrm{kpc}$ (Sridharan et al. 2002) implies that the linear radius of the ring distribution is $980 \mathrm{AU}$.

G26.598-00.024. The maser is located $0{ }^{\prime} 85$ from a cometary H II region (Fig. 2) with a spectral index of 0.23 between 1.4 and $5 \mathrm{GHz}$ (Becker et al. 1994). This corresponds to linear distances of 1530(11400) AU. The flux density of $4.4 \mathrm{mJy}$ at $8.4 \mathrm{GHz}$, compared with $55 \mathrm{mJy}$ at $5 \mathrm{GHz}$, implies that the turnover frequency is near $5 \mathrm{GHz}$. The methanol maser probably forms behind a shock front induced by the H II region. The strongest maser component, at $24.2 \mathrm{~km} \mathrm{~s}^{-1}$, coincides in velocity with a $24.3 \mathrm{~km} \mathrm{~s}^{-1}$ absorption feature $(-59.8 \mathrm{mJy})$ in the $\mathrm{H}_{2} \mathrm{CO}$ line at $4.8 \mathrm{GHz}$ (Sewilo et al. 2004).

G36.115+00.552. The brightest component of the NW maser structure is 0.2 away from the weak point continuum source at $\mathrm{PA}=120^{\circ}$ (Fig. 2). The shape of this complex suggests the existence of an outflow. However, the kinematics are not consistent with an outflow model (Sect. 4.2.3), nor do CO (2-1) line maps at $29^{\prime \prime}$ resolution detect any molecular outflow (Zhang et al. 2005).

\section{Discussion}

\subsection{Kinematic models of the origins of methanol masers}

The diverse morphologies of methanol masers indicate that there is no straighforward explanation of the origin of this emission, as discussed previously (Norris et al. 1993; Phillips et al. 1998; Minier et al. 2000). The main hypotheses for the origin of methanol masers assume that they originate in a circumstellar disc or torus, in outflows or a shock colliding with a rotating molecular cloud. The results that we obtained by applying the existing models to our data are summarized below.

\subsubsection{Rotating and expanding ring}

Ring-like masers, which are prevalent in the present sample, may represent an inclined disc or torus around a massive protostar or young star. There is a tendency for flatter structures to have a larger velocity width, in all sources apart from G23.657-00.127, (see Table 5), which suggests that we observe the effects of inclination and all motion is intrinsic to the plane of the ring. To test this hypothesis, we applied the model of a rotating and expanding thin disc (Uscanga et al. 2008) to the nine ring-like masers. First, the coordinates of the spots $\left(x_{j}, y_{j}\right)$ were transformed to a reference system $\left(x_{j}^{\prime}, y_{j}^{\prime}\right)$ in which the origin was the center of the ellipse and the $x^{\prime}$-axis was directed along the major axis of the projected ellipse (see Fig. 1 in Uscanga et al. 2008). We then attempted to reproduce the kinematics using the LSR velocities $\left(V_{\mathrm{LSR}}\right)$ of the maser spots to determine rotation $\left(V_{\text {rot }}\right)$, expansion $\left(V_{\text {exp }}\right)$, and systemic $\left(V_{\text {sys }}\right)$ velocities of each source. The solutions were based on the minimisation of the $\chi_{\mathrm{V}}^{2}$ function expressed in Eq. (8) by Uscanga et al. (2008)

$$
\chi_{V}^{2}=\frac{1}{N-3} \sum_{j=1}^{N} \frac{1}{\sigma_{V}^{2}}\left(V_{\mathrm{LSR}, j}-V_{\mathrm{sys}}-\frac{x_{j}^{\prime}}{a} V_{\mathrm{rot}} \sin i-\frac{y_{j}^{\prime}}{a} V_{\exp } \tan i\right)^{2},
$$

where $\sigma_{\mathrm{V}}$ was the spectral resolution of the observations corresponding to the uncertainty in the LSR velocity. The inclination angle is the angle between the line-of-sight and the normal to the ring plane, which is defined to be $i=\operatorname{acos}\left(\frac{b}{a}\right)$. The semimajor and semi-minor axes $(a, b)$ were taken from Table 8 . We note, that we cannot determine the sign of the inclination angle from the data available and the direction of the rotation is therefore ambiguous, nor distinguish between outflow and contraction. Additional information (e.g., spectroscopic and interferometric observations of molecular clouds at mas resolution) are necessary to solve these questions. For the purpose of the model, we assumed that the brightest and the most complex half of the ellipse is closer to the observer. The results of fitting are 

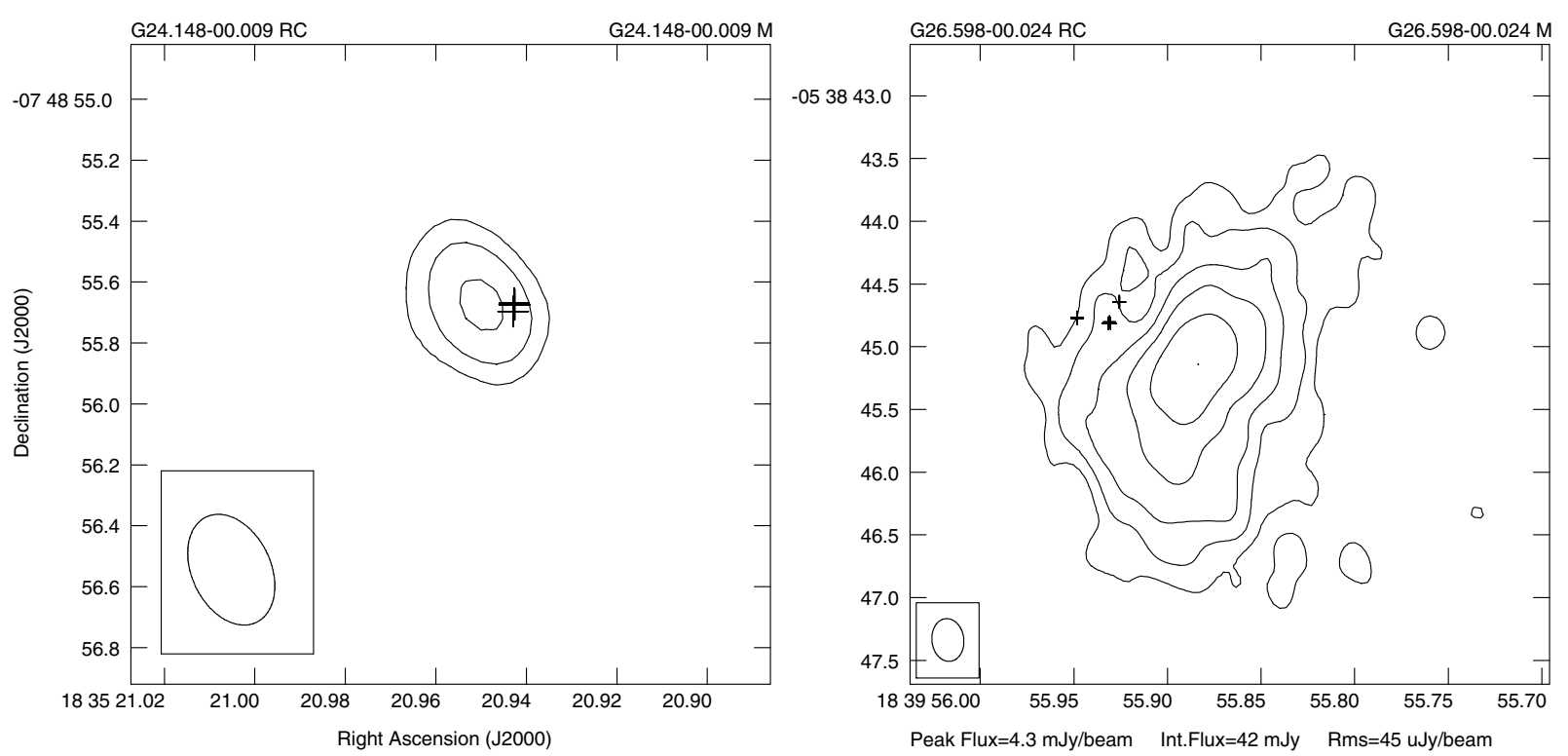

Peak Flux $=1.05 \mathrm{mJy} /$ beam Int.Flux=1 mJy Rms=73 uJy/beam
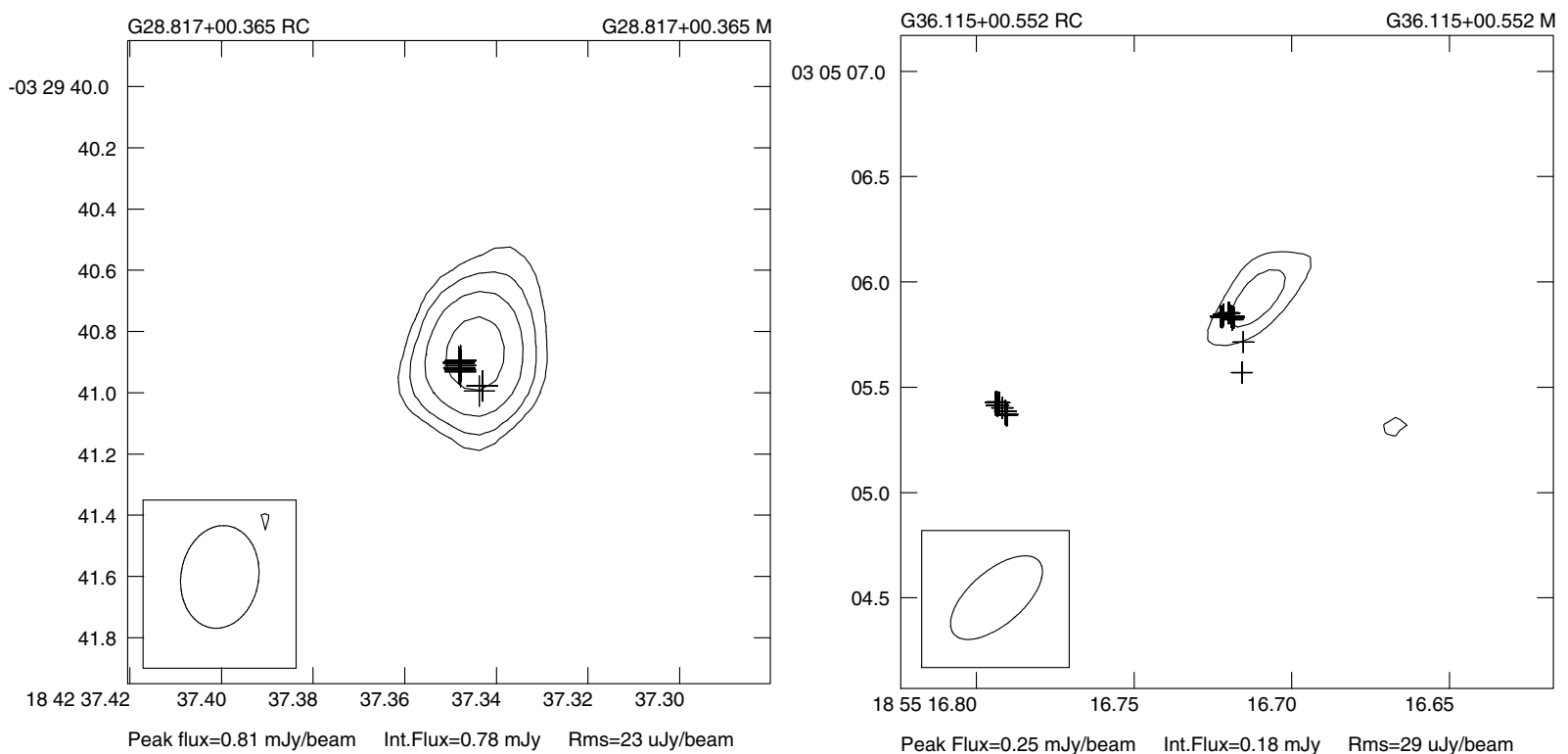

Fig. 2. The $8.4 \mathrm{GHz}$ continuum emission towards four methanol maser sites taken using VLA on 2007 August 18. The names of radio continuum sources (RC) relate to the Galactic coordinates of their peak fluxes. The peak and integrated fluxes as well as the levels of rms $\left(1 \sigma_{\mathrm{rms}}\right)$ are given under each map and the beamsizes are presented at the left bottom corners. Contours trace the levels of $3 \sigma_{\text {rms }} \times(1,2,4,8,16,32,64, \ldots)$. Crosses represent the $6.7 \mathrm{GHz}$ methanol maser spots registered using EVN.

summarized in Table 9 and an example of the fit of the model to the data, for G33.980-00.019, is presented in Fig. 4.

We note that in general the expansion/infall velocity is higher than the rotation component in the majority of sources, as can be clearly seen in the maser spot distributions (Fig. 1). If the rotation velocity was instead higher than that of expansion or infall, the extreme values of velocities could be produced where the major axis and ellipse intersect (at a position angle of $0^{\circ}$ from the major axis). In four of nine sources, the opposite result is found that higher blue- or red-shifted velocities appear where the minor axes intersect the ellipses (see plots of G23.207-00.377, G23.389+00.185, G24.634-00.324, and G25.411+00.105). The average position angle of the most extreme velocity with respect to the major axis in all nine rings, and the standard dispersion in the mean, is $52^{\circ} \pm 11^{\circ}$. This suggests that the masers originate in the zone where the radial motions exist and expansion/infall plays a role. This could occur at the interface between the disc/torus and outflow. A similar result was reported for the archetypical object Cep A (Torstensson et al. 2009), where the major axis of the elliptical distribution of $6.7 \mathrm{GHz}$ methanol masers is perpendicular to the bipolar outflow. Their LSR velocity distributions show similar characteristics in that expansion or contraction dominates over the rotation.

\subsubsection{Linear maser as edge-on disc?}

A thin disc seen edge-on would appear to have a linear morphology. Norris et al. (1998) argued that maser radiation propagates most strongly in the plane of the disc due to the greater column depth, to explain why so many methanol masers with linear morphology appeared in the data then available. 
Table 9. Parameters derived by fitting kinematics of the rotating and expanding disc model.

\begin{tabular}{lrrccc}
\hline \hline Source & $\begin{array}{r}V_{\text {rot }} \\
\left(\mathrm{km} \mathrm{s}^{-1}\right)\end{array}$ & $\begin{array}{r}V_{\text {exp }} \\
\left(\mathrm{km} \mathrm{s}^{-1}\right)\end{array}$ & $\begin{array}{c}V_{\text {sys }} \\
\left(\mathrm{km} \mathrm{s}^{-1}\right)\end{array}$ & $\begin{array}{c}i \\
\left({ }^{\circ}\right)\end{array}$ & $\chi_{\mathrm{V}}^{2}$ \\
\hline G23.207-00.377 & -1.17 & +3.96 & 79.46 & -69 & 168 \\
G23.389+00.185 & -1.26 & -1.71 & 74.91 & +54 & 101 \\
G23.657-00.127 & +7.29 & -2.61 & 81.90 & +16 & 641 \\
G24.634-00.324 & +8.64 & -2.25 & 38.96 & +71 & 544 \\
G25.411+00.105 & +0.09 & -1.17 & 95.84 & +47 & 206 \\
G26.598-00.024 & +0.81 & -0.81 & 24.58 & -55 & 30 \\
G31.047+00.356 & $+4 \times 10^{-7}$ & -2.43 & 80.68 & -61 & 211 \\
G33.980-00.019 & -0.45 & +2.97 & 61.86 & -62 & 123 \\
G34.751-00.093 & -1.17 & -2.88 & 51.17 & +53 & 22 \\
\hline
\end{tabular}

The signs + and - of the rotation and expansion velocities refer to the clockwise or anti-clockwise rotation and outflow or inflow for positive $i$. Both rotation and flow are reversed in a case of negative $i$. For each source, both signs together with the sign of $i$ could be reversed since our model does not give the directions unambiguously.

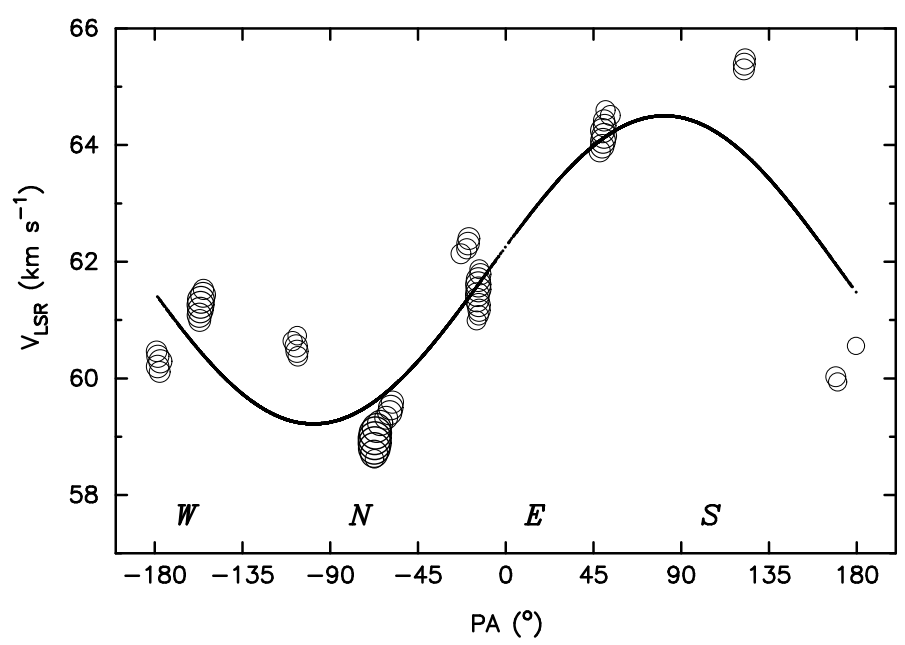

Fig. 4. Velocity of the maser spots in G33.980-00.019 versus azimuth angle measured from the major axis (north to east). The open circles represent the data and are proportional to the logarithm of the flux densities. The sinusoidal line represents the best-fit kinematical model of a rotating and expanding disc (with infintesimal thickness) using the parameters listed in Table 9.

We do not confirm this selection effect and note that the increased sensitivity detects more complex structures of masing regions. Only $16 \%$ of our sample are linear masers (compared to $29 \%$ ring-like masers) and they are also not the brightest, although it is possible that if there is strong expansion or infall, this would produce a steeper velocity gradient in edge-on discs and possibly make the masers fainter.

We calculated the mass that each linear maser structure would contain if it originated in a disc in Keplerian rotation, using a method similar to that of Minier et al. (2000). Assuming that the masing area corresponds to the diameter of the disc, the average central mass of these five linear structures is $0.12 M_{\odot}$ or $0.44 M_{\odot}$ for the near or far kinematic distances, respectively. These subsolar values are very unlikely for massive protostars. We agree with Minier et al. (2000) that the underlying assumption is wrong and we do not detect the full diameter of the disc. However, if we assume that the true extent of the linear masers is similar to the average size of the major axis of the nine ellipses (188 mas), this implies a mean central mass of $76 M_{\odot}$ or $190 M_{\odot}$ for the near or far kinematic distance, respectively. These values seem unrealistically high. Another solution is that the masers are not bound by Keplerian rotation and we argue that the most likely explanation is that the linear morphology results from a different scenario. Linear structures with ordered velocity gradients may be produced readily by geometric effects in outflows. It seems significant that Szymczak et al. (2007) detected molecular line emission from $\mathrm{HCO}^{+}, \mathrm{CO}$, and CS towards these five sources using the IRAM $30 \mathrm{~m}$ telescope, supporting the outflow scenario. In addition, De Buizer et al. (2009) imaged $\mathrm{SiO}$ outflows towards five methanol masers with linear morphologies. They found that the spatial orientations of the outflows were inconsistent with the methanol masers tracing discs. Linear masers produced by outflows seemed to provide a much more plausible scenario. Finally, we also note that the linear masers have a smaller extent than most other maser structures (Table 5). In particular, the entire G35.793-00.175 structure is only 10 mas long corresponding to the typical size of an individual maser cluster in other sources. We conclude that most of the linear masers are more likely to be associated with outflows than with edge-on discs, although it is possible that more sensitive observations might indicate that some are part of ring-like or other structures. G25.411+00.105 (see Sect. 3.4) provides such an example, since Beuther et al. (2002) found only two maser spots, whilst in the present study we detected 30 spots, forming a ring-like morphology.

\subsubsection{Propagating shock front}

Dodson et al. (2004) proposed another model for linear methanol masers. A low speed planar shock propagating through the rotating molecular cloud would produce a linear spatial distribution of maser spots if the shock was propagating close to perpendicular to the line of sight. The linearity would be distrupted where the shock interacted with density perturbations in the starforming regions. The main diagnostic for this scenario is the perpendicular orientation of velocity gradients within individual clusters with respect to the main large-scale velocity gradient. We analyzed the internal gradients of clusters and found this behaviour in three out of five masers with linear morphologies (G22.335-00.155, G23.966-00.109, and G30.318+00.070). In addition, the arched source, G33.641-00.228, shows similar characteristics in four (out of six) clusters, which have internal velocity gradients perpendicular to the longest axis of the overall structure. All these masers have young massive objects in close proximity less then 1'.22 away (Sect. 4.2), which could be responsible for the external shocks. Proper motions studies are needed to verify this scenario.

\subsubsection{Bipolar outflow}

The bipolar outflow model for $\mathrm{H}_{2} \mathrm{O}$ masers associated with a high-mass young stellar object was proposed by Moscadelli et al. (2000) and confirmed in IRAS20126+4104 by proper motion studies (Moscadelli et al. 2005). We also tested this model for all sources in this study. The assumptions of the model are as follows: masers originate in the surface of a conical bipolar jet due to the interaction between the ionised jet and the surrounding neutral medium, and the velocity of a maser spot, $V_{\mathrm{o}}$, is directed radially outward from the central star at a constant value. The center of the coordinate system is at the vertex of the cone, the $z$-axis is along the line of sight, and the $x$-axis coincides with 
Table 10. Parameters derived from fitting the biconical outflow model of Moscadelli et al. (2000).

\begin{tabular}{lcccccc}
\hline \hline Source & $\begin{array}{c}\Delta \text { RA, } \Delta \text { Dec. }{ }^{*} \\
\text { (mas, mas) }\end{array}$ & $\begin{array}{c}V_{\mathrm{o}} \\
\left(\mathrm{km} \mathrm{s}^{-1}\right)\end{array}$ & $\begin{array}{c}\text { PA } \\
\left({ }^{o}\right)\end{array}$ & $\begin{array}{c}\Psi \\
\left({ }^{o}\right)\end{array}$ & $\begin{array}{c}\theta \\
\left({ }^{o}\right)\end{array}$ & $\chi^{2}$ \\
\hline G23.707-00.198 & 33,45 & -52.7 & 57 & 115 & 37 & 1.2 \\
G24.541+00.312 & $-52,-15$ & -15.5 & 112 & 52 & 48 & 2.4 \\
\hline
\end{tabular}

* Coordinates relative to the brightest spots as listed in Table 5.

the projection of the outflow on the plane of the sky (see Fig. 4 in Moscadelli et al. (2000)). Taking the central velocity of the maser emission $\left(V_{\mathrm{c}}\right)$ to be the systemic LSR velocity, we minimized the $\chi^{2}$ function as expressed in Eq. (3) of Moscadelli et al. (2000)

$\chi^{2}=\sum_{j=2}^{n}\left(\frac{V_{z}^{j}}{V_{z}^{1}}-\frac{V_{\mathrm{LSR}}^{j}-V_{\mathrm{c}}}{V_{\mathrm{LSR}}^{1}-V_{\mathrm{c}}}\right)^{2}$,

where $n$ is the number of spots, $V_{\mathrm{z}}^{\mathrm{j}}$ is the velocity of the spot $\# \mathrm{j}$ calculated using Eqs. (1) and (2) from Moscadelli et al. (2000), and $V_{\text {LSR }}^{j}$ is the observed LSR velocity of the spot.

We obtained reasonable fits for only two of the arched sources, G23.707-00.198 and G24.541+00.312. The following best-fit model parameters are listed in Table 10: the position of the vertex, the opening angle of the cone $(2 \theta)$, the inclination angle between the outflow axis and the z-axis $(\psi)$, and the direction of the $x$-axis, PA, which is the position angle of the outflow on the plane of the sky. A comparison between the modelled and observed data and a sketch of the orientation of the outflow was presented previously in Fig. 1 in Bartkiewicz et al. (2006). It is significant that the vertices of the cones calculated for both sources coincide with infrared sources within the position uncertainties (Sect. 4.2). In addition, molecular line emission at similar LSR velocities was reported towards both sources (Szymczak et al. 2007). As we mentioned previously, Araya et al. (2006) imaged $\mathrm{H}_{2} \mathrm{CO}$ maser emission at $4.8 \mathrm{GHz}$ towards $\mathrm{G} 24.541+00.312$. All of these findings ensure that the outflow scenario is plausible for these two objects.

We tested the outflow model intensively on the four maser sources associated with HII regions. However, we did not achieve a reasonable fit that would place the vertex of the cone at the center of the radio continuum object, whilst reproducing the maser spot kinematics, for any of these sources.

\subsection{Association with MIR emission}

Early work by Szymczak et al. (2002) showed that $~ 80 \%$ of methanol masers have infrared counterparts in IRAS and/or MSX catalogues. Since maser data with position accuracy as good or better than these IR catalogues $\left(30^{\prime \prime}-5^{\prime \prime}\right)$ have become available, the fraction of secure identifications has diminished (Pandian et al.2007). We can now attempt identifications with sub-arcsec resolution data.

We used Spitzer IRAC data to test the association between methanol masers and MIR emission. Images at 3.6, 4.5, 5.8, and $8.0 \mu \mathrm{m}$ from the GLIMPSE survey, all at 0 .' $^{\prime} 6$ resolution (Fazio et al. 2004), were retrieved from the Spitzer archive ${ }^{3}$ and compared with the radio data using AIPS.

${ }^{3}$ http://irsa.ipac.caltech.edu/applications/Cutouts/

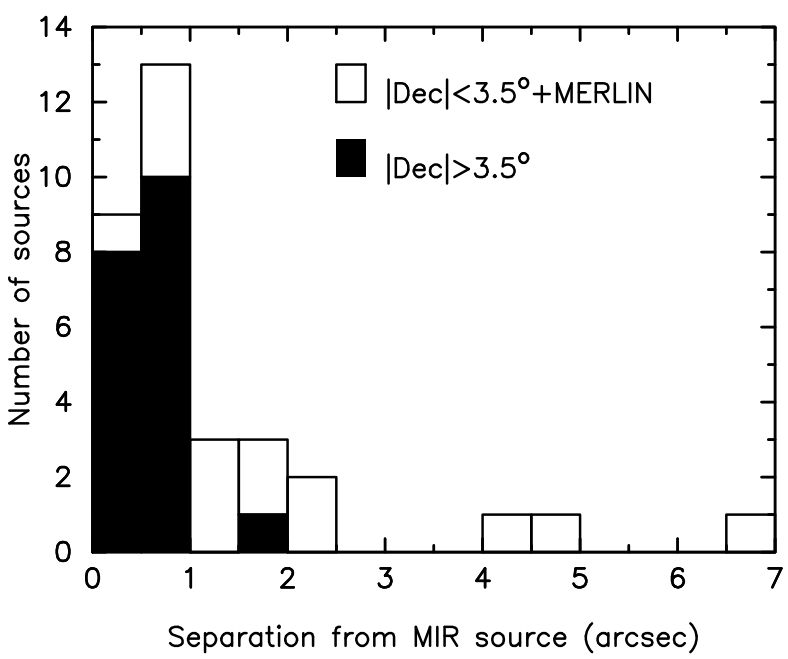

Fig. 5. Histogram of number of the methanol masers as a function of angular separation from associated MIR sources at $4.5 \mu \mathrm{m}$. Bin size is 0 .'5. The sources are divided into two groups with position measurements of high accuracy ( $\mid$ Dec. $\mid>3.5$ ) and lower accuracy ( $\mid$ Dec. $\mid<3.5$ plus those measured with MERLIN alone).

The angular separation between the brightest maser spot of each source in our sample and the nearest MIR source at $4.5 \mu \mathrm{m}$ $\left(\Delta_{\text {MIR }}\right)$ is given in Table 5 and a histogram of the results is shown in Fig. 5. The average separation for the entire sample is $1^{\prime \prime} .18 \pm 0 . \prime 19$ and the median value is 0.89 , whereas, for the subsample of 19 objects at |Dec.| $>3.5$ with coordinates measured using the EVN, the corresponding values are 0.'61 \pm 0. '09 and $0.0^{\prime} 51$, respectively. The remaining 14 targets with $\mid$ Dec. $\mid<3.5$, or with positions derived from the MERLIN data alone, show a larger $\Delta_{\text {MIR }}$ with the mean and median values of $11^{\prime \prime} 96 \pm 0.35$ and 1.'61, respectively. Since the images of these sources had poor $u v$-plane coverage and hence less accurate astrometry, we suggest that their higher estimates of $\Delta_{\text {MIR }}$ have a higher uncertainty and that most associations are also likely to be genuine.

We conclude that the majority of maser sources coincide, within $\lesssim 1^{\prime \prime}$ with MIR emission, i.e., the maser emission from each source falls within one Spitzer pixel (nominal size $\sim 1^{\prime \prime} .2$, see Fazio et al. 2004) in the IRAC $4.5 \mu \mathrm{m}$ image. This strongly reinforces the finding by Cyganowski et al. (2008), who reported coincidences to within $\lesssim 5^{\prime \prime}$ for 46 out of 64 methanol sources with positions measured using the ATCA. Extended emission in the IRAC $4.5 \mu \mathrm{m}$ band has been postulated to be a tracer of shocked molecular gas in protostellar outflows (e.g., Davies et al. 2007; Cyganowski et al. 2008 and references therein). This IRAC band contains $\mathrm{H}_{2}$ and $\mathrm{CO}$ lines that may be excited by shocks. Using shock models, Smith \& Rosen (2005) predicted that $\mathrm{H}_{2}$ emission in outflows is 5-14 times stronger in the $4.5 \mu \mathrm{m}$ band than in the $3.6 \mu \mathrm{m}$ band. Cyganowski et al. (2008) identified more than 300 objects with extended $4.5 \mu \mathrm{m}$ emission, which may relate to outflows in massive stars. Four maser sources from our sample, G23.966-00.109, G35.793-00.175, G37.479-00.105, and G39.100+00.491, were included in their catalogue.

In order to search for extended emission, we created images of the $4.5 \mu \mathrm{m}-3.6 \mu \mathrm{m}$ excess by subtracting the $3.6 \mu \mathrm{m}$ image of each of our sources from its $4.5 \mu \mathrm{m}$ counterpart, and compared these with the $4.5 \mu \mathrm{m}$ image. All the sources have extended $4.5 \mu \mathrm{m}$ emission. Figure 6 shows the $4.5 \mu \mathrm{m}-3.6 \mu \mathrm{m}$ excess superimposed on the $4.5 \mu \mathrm{m}$ image for selected sources. Source G21.407-00.254 illustrates how the maser emission 
located precisely inside the brightest pixel of the $4.5 \mu \mathrm{m}-3.6 \mu \mathrm{m}$ image outside the $4.5 \mu \mathrm{m}$ peak. There is also evidence that maser clusters in at least three sources are associated with $4.5 \mu \mathrm{m}$ emission excess. The image at the position of G24.148-00.009 shows that the maser coincides exactly with maxima of both the $4.5 \mu \mathrm{m}-3.6 \mu \mathrm{m}$ excess and of the $4.5 \mu \mathrm{m}$ emission. Weak, extended $4.5 \mu \mathrm{m}$ emission is also seen at the edges of two neighbouring sources and in diffuse lanes. The maser G37.598+00.425 coincides with a maximum in a very asymmetric distribution of $4.5 \mu \mathrm{m}-3.6 \mu \mathrm{m}$ emission excess that is displaced by $\sim 1$ '. 5 from a peak of $4.5 \mu \mathrm{m}$ emission, implying that the methanol maser and the excess in the $4.5 \mu \mathrm{m}$ IRAC band are very strongly related. Similar evidence is provided by G38.203-00.067, where the maser is offset by more than $8^{\prime \prime}$ from a bright MIR object. The maser is $\sim 1$ !' 7 away from a weak bump in a large lane of diffuse $4.5 \mu \mathrm{m}-3.6 \mu \mathrm{m}$ emission excess. Inspection of IRAC images for other bands suggests that the maser is probably associated with a faint MIR object.

All the counterparts in the sample have MIR properties typical of embedded young massive objects (e.g., Kumar et al. 2007) associated with the methanol masers (Ellingsen 2006). We defer a detailed discussion of the MIR spectral indices of individual objects, because several bright sources (e.g., G23.389+00.185, G23.657-00.127, and G24.634-00.324) are saturated in the IRAC images, while for others only upper limits to the $3.6 \mu \mathrm{m}$ flux can be derived (e.g., G23.966-00.109, G37.030-00.039, and G38.203-00.067). We note only, that the MIR objects associated with methanol masers that have a ring-like morphology have much stronger emission at $8 \mu \mathrm{m}$, and all the objects that are saturated in the IRAC images have regular maser structures. In contrast, no object saturated in the IRAC images has a complex maser morphology. This suggests that the MIR counterparts of masers with less regular structures are deeply embedded massive stars that are younger than the counterparts of those with more regular, ring-like maser structures. One can speculate that in those younger objects the methanol maser originates in a limited number of confined regions, whilst more regular structures emerge during later evolutionary stages.

In summary, we have found strong evidence of a close coincidence of $6.7 \mathrm{GHz}$ methanol masers with $4.5 \mu \mathrm{m}$ emission excess. This provides a firm argument that methanol emission originates in those inner parts of an outflow or disc/torus where the molecular gas is shocked.

This hypothesis is strongly supported by the kinematic model that can be successfully applied to the ring masers in our sample, which involved a rotating and expanding disc wherein expansion dominates the velocity field. Moreover, a fraction of masers seem to fit the model of a bipolar jet or that of a shock front colliding with the surrounding molecular material.

\section{Conclusions}

We have completed a $6.7 \mathrm{GHz}$ methanol line imaging survey of 33 maser sources in the Galactic plane. High quality EVN images were obtained for 31 targets showing their mas-scale structures, from which we derived the absolute positions of 29 sources with a few mas accuracy. In most cases, the masers exhibit complex structures. The observed morphologies can be divided into five groups: simple, linear, ring-like, complex, arched, and pair. It is surprising that about $29 \%$ of the sources exhibit ring-like distributions of maser spots that were not apparent in previous VLBI surveys, which were less sensitive and concentrated on brighter masers. We find that many of the other ordered structures, notably linear and arched, can be interpreted as originating from the interaction between collimated or biconical outflows and the surrounding medium.

A simultaneous survey at high angular resolution of continuum emission at $8.4 \mathrm{GHz}$ towards the maser targets revealed that only $16 \%$ of the methanol masers appear to be physically related to H II regions. In three cases, the maser coincides with a peak in a compact and relatively weak continuum object. These are believed to be young UC H II regions. In general, these results seem to imply that $6.7 \mathrm{GHz}$ methanol masers appear before the ionised region is detectable at $\mathrm{cm}$ wavelengths. The hypothesis that these masers are possible associated with $\mathrm{HC} \mathrm{H}$ II regions needs to be verified.

We used the Spitzer GLIMPSE survey to demonstrate that the majority of methanol masers are closely associated with MIR emission. Analyzis of the MIR counterparts suggests that masers with a regular, ring-like morphology are associated with more evolved proto- or young stars, relative to the counterparts of masers with more complex, irregular structures. Moreover, both the kinematics of the masers with a ring-like distribution and the characteristics of associated MIR emission are consistent with a scenario in which the methanol emission is produced by shocked material associated with a disk or torus, possibly from an interaction with the outflow.

Acknowledgements. Our special acknowledgements to Dr. Peter Thomasson at Jodrell Bank Observatory and to Dr. Bob Campbell at JIVE for detailed support in many stages of this project. We also thank Dr. Riccardo Cesaroni, Dr. Luca Moscadelli, Dr. Lucero Uscanga, Dr. Krzysztof Goździewski, Dr. Piotr Nieżurawski and Kalle Torstensson for useful discussions. This work was benefited from the Polish MNiI grant 1P03D02729 and from research funding from the EC 6th Framework Programme.

The European VLBI Network (EVN) is a joint facility of European, Chinese, South African and other radio astronomy institutes funded by their national research councils. MERLIN is a National Facility operated by the University of Manchester at Jodrell Bank Observatory on behalf of STFC. The Very Large Array (VLA) of the National Radio Astronomy Observatory is a facility of the National Science Foundation operated under cooperative agreement by Associated Universities, Inc. This research has made use of the NASA/IPAC Infrared Science Archive, which is operated by the Jet Propulsion Laboratory, California Institute of Technology, under contract with the National Aeronautics and Space Administration.

\section{References}

Araya, E., Hofner, P., Goss, W. M., et al. 2006, ApJ, 643, L33

Bartkiewicz, A., Szymczak, M., \& van Langevelde, H. J. 2004, PoS, Proceeding of the 7th European VLBI Network Symposium, ed. R. Bachiller, F. Colomer, J. F. Desmurs, \& P. de Vincente, Toledo, Spain, 187

Bartkiewicz, A., Szymczak, M., \& van Langevelde, H. J. 2005, A\&A, 442, L61 Bartkiewicz, A., Szymczak, M., \& van Langevelde, H. J. 2006, PoS, Proceedings of the 8th European VLBI Network Symposium, ed. A. Marecki, 039

Bartkiewicz, A., Brunthaler, A., Szymczak, M., van Langevelde, H. J., \& Reid, M. J. 2008, A\&A, 490, 787

Bartkiewicz, A., Szymczak, M., \& van Langevelde, H. J. 2009, PoS, Proceedings of the 9th European VLBI Network Symposium, ed. F. Montovani, 037

Becker, R. H., White, R. L., Helfand, D. J., \& Zoonematkermani, S. 1994, ApJS, 91, 347

Beuther, H., Walsh, A., Schilke, P., et al. 2002, A\&A, 390, 289

Caswell, J. L., Vaile, R. A., Ellingsen, S. P., Whiteoak, J. B., \& Norris, R. P. 1995, MNRAS, 272, 96

Cragg, D. M., Sobolev, A. M., \& Godfrey, P. D. 2002, MNRAS, 331, 521

Cyganowski, C. J., Whitney, B. A., Holden, E., et al. 2008, AJ, 136, 2391

Dartois, E., Schutte, W., Geballe, T. R., et al. 1999, A\&A, 342, L32

De Buizer, J. M., \& Minier, V. 2005, ApJ, 628, L151

De Buizer, J. M., Redman, R. O., Longmore, S. N., Caswell, J., \& Feldman, P. A. 2009, A\&A, 493, 127

Davis, C. J., Kumar, M. S. N., Sandell, G., et al. 2007, MNRAS, 374, 29

Diamond, P. J., Garrington, S. T., Gunn, A. G., et al. 2003, MERLIN USer Guide, Ver. 3

Dodson, R., Ojha, R., \& Ellingsen, S. P. 2004, MNRAS, 351, 779

Ellingsen, S. P. 2006, ApJ, 638, 241 
Fazio, G. G., Hora, J. L., Allen, L. E., et al. 2004, ApJS, 154, 10

Fitzgibbon, A., Pilu, M., \& Fisher, R. B. 1999, IEEE Transactions on Pattern Analysis and Machine Intelligence, 21, 476

Kumar, M. S. N., \& Grave, J. M. C. 2007, A\&A, 472, 155

Longmore, S. N., Burton, M. G., Barnes, P. J., et al. 2007, MNRAS, 379, 535

Menten, K. M. 1991, ApJ, 380, L75

Menten, K. M., Reid, M. J., Pratap, P., Moran, J. M., \& Wilson, T. L. 1992, ApJ, 401, L39

Minier, V., Booth, R. S., \& Conway, J. E. 2000, A\&A, 362, 1093

Moscadelli, L., Cesaroni, R., \& Rioja, M. J. 2000, A\&A, 360, 663

Moscadelli, L., Menten, K. M., Walmsley, C. M., \& Reid, M. J. 2002, ApJ, 564, 813

Moscadelli, L., Cesaroni, R., \& Rioja, M. J. 2005, A\&A, 438, 889

Norris, R. P., Whiteoak, J. B., Caswell, J. L., Wieringa, M. H., \& Goigh, R. G. 1993, ApJ, 412, 222

Norris, R. P., Byleveld, S. E., Diamond, P. J., et al. 1998, ApJ, 508, 275

Pandian, J. D. \& Goldsmith, P. F. 2007, ApJ, 669, 435

Pestalozzi, M. R., Elitzur, M., Conway, J. E., \& Booth, R. S. 2004, ApJ, 603, L113
Philips, C. J., Norris, R. P., Ellingsen, S. P., \& McCulloch, P. M. 1998, MNRAS, 300,1131

Sewilo, M., Watson, C., Araya, E., et al. 2004, ApJS, 154, 553

Smith, M. D., \& Rosen, A. 2005, MNRAS, 357, 1370

Sridharan, T. K., Beuther, H., Schilke, P., Menten, K. M., \& Wyrowski, F. 2002, ApJ, 566, 931

Szymczak, M., Hrynek, G., \& Kus, A. J. 2000, A\&ASS, 143, 269

Szymczak, M., Kus, A. J., Hrynek, G., Kepa, A., \& Pazderski, E. 2002, A\&A, 392, 277

Szymczak, M., Pillai, T., \& Menten, K. M. 2005, A\&A, 434, 613

Szymczak, M., Bartkiewicz, A., \& Richards, A. M. S. 2007, A\&A, 468, 617

Uscanga, L., Gomez, Y., Raga, A. C., et al. 2008, MNRAS, 390, 1127

Torstensson, K., van Langevelde, H. J., Vlemmings, W., \& van der Tak, F. 2009, PoS, Proceedings of the 9th European VLBI Network Symposium, ed. F. Montovani, 39

Walsh, A. J., Burton, M. G., Hyland, A. R., \& Robinson, G. 1998, MNRAS, 301, 640

van der Walt, D. J., Sobolev, A. M., \& Butner, H. 2007, A\&A, 464, 1015

Zhang, Q., Hunter, T. R., Brand, J., et al. 2005, ApJ, 625, 864 
A. Bartkiewicz et al.: The diversity of methanol maser morphologies from VLBI, Online Material $p 1$

Table 1. Summary of previous high angular resolution studies of $6.7 \mathrm{GHz}$ methanol masers.

\begin{tabular}{|c|c|c|c|c|c|c|}
\hline Survey & Telescope & $\begin{array}{l}\text { Spectral resolution } \\
\left(\mathrm{km} \mathrm{s}^{-1}\right)\end{array}$ & $\begin{array}{l}\text { Angular resolution } \\
\text { (mas) }\end{array}$ & $\begin{array}{c}1 \sigma_{\mathrm{rms}} \\
\left(\mathrm{mJy}^{-1} \text { beam }^{-1}\right)\end{array}$ & Number of targets & $\begin{array}{l}\text { Median peak flux density } \\
\text { (Jy) }\end{array}$ \\
\hline Norris et al. (1993) & ATCA & 0.3 & 1500 & $\sim 500$ & 15 & 475 \\
\hline Phillips et al. (1998) & ATCA & 0.35 & 1500 & $\sim 50$ & 33 & 41 \\
\hline Walsh et al. (1998) & ATCA & 0.18 & 1500 & 300 & 97 & 23 \\
\hline Minier et al. (2000) & EVN & 0.04 & few & $-^{*}$ & 14 & 272 \\
\hline Dodson et al. (2004) & LBA & 0.2 & 5.3 & 2 & 5 & 148 \\
\hline This paper & EVN & $0.09 ; 0.18$ & $\sim 6 \times 14$ & $3-12$ & 31 & 3.6 \\
\hline
\end{tabular}

* The value not given. 
A. Bartkiewicz et al.: The diversity of methanol maser morphologies from VLBI, Online Material p 2

Table 2. Sample of methanol masers observed with the EVN. The names are the Galactic coordinates of the brightest spot of each target obtained in post-processing EVN data. The dates of each observing run are listed in Table 4. The phase-calibrator names and angular separations from the targets are given.

\begin{tabular}{|c|c|c|c|}
\hline $\begin{array}{c}\text { Source } \\
\text { Gll.1ll } \pm \text { bb.bbb }\end{array}$ & $\begin{array}{l}\text { Observing } \\
\text { run }\end{array}$ & Phase-calibrator & $\begin{array}{c}\text { Separation } \\
\left(\begin{array}{c}\circ \\
\text {.) }\end{array}\right.\end{array}$ \\
\hline G21.407-00.254 & $3 a$ & J1825-0737 & 3.1 \\
\hline G22.335-00.155 & $3 \mathrm{a}$ & J1825-0737 & 2.5 \\
\hline $\mathrm{G} 22.357+00.066^{1}$ & $4 a$ & J1825-0737 & 2.3 \\
\hline G23.207-00.377 & 2 & J1825-0737 & 2.6 \\
\hline G23.389+00.185 & 2 & J1825-0737 & 2.1 \\
\hline \multirow{3}{*}{ G23.657-00.127 } & 2 & J1825-0737 & 2.4 \\
\hline & $3 a$ & J1825-0737 & 2.4 \\
\hline & $4 a$ & $\mathrm{~J} 1825-0737$ & 2.4 \\
\hline \multirow[t]{2}{*}{ G23.707-00.198 } & 2 & $\mathrm{~J} 1825-0737$ & 2.5 \\
\hline & $3 \mathrm{a}$ & J1825-0737 & 2.5 \\
\hline G23.966-00.109 & $3 a$ & $\mathrm{~J} 1825-0737$ & 2.5 \\
\hline G24.148-00.009 & $3 a$ & $\mathrm{~J} 1825-0737$ & 2.4 \\
\hline $\mathrm{G} 24.541+00.312$ & 2 & $\mathrm{~J} 1825-0737$ & 2.4 \\
\hline G24.634-00.324 & $4 a$ & $\mathrm{~J} 1825-0737$ & 2.9 \\
\hline $\mathrm{G} 25.411+00.105^{2}$ & $4 a$ & $\mathrm{~J} 1825-0737$ & 3.1 \\
\hline G26.598-00.024 & $4 a$ & $\mathrm{~J} 1825-0737$ & 4.1 \\
\hline G27.221+00.136 & 2 & $\mathrm{~J} 1825-0737$ & 4.5 \\
\hline G28.817+00.365 & $4 \mathrm{~b}$ & J1834-0301 & 2.2 \\
\hline G30.318+00.070 & $4 \mathrm{~b}$ & J1834-0301 & 3.2 \\
\hline G30.400-00.296 & $4 \mathrm{~b}$ & $\mathrm{~J} 1834-0301$ & 3.5 \\
\hline G31.047+00.356 & $4 \mathrm{~b}$ & J1834-0301 & 3.5 \\
\hline G31.156+00.045 & $4 \mathrm{~b}$ & J1834-0301 & 3.8 \\
\hline G31.581+00.077 & $4 \mathrm{~b}$ & $\mathrm{~J} 1834-0301$ & 4.1 \\
\hline $\mathrm{G} 32.992+00.034^{2}$ & $4 \mathrm{c}$ & $\mathrm{J} 1907+0127$ & 4.2 \\
\hline G33.641-00.228 & 1 & $\mathrm{~J} 1907+0127$ & 3.5 \\
\hline G33.980-00.019 & $4 c$ & $\mathrm{~J} 1907+0127$ & 3.5 \\
\hline G34.751-00.093 & $4 c$ & $\mathrm{~J} 1907+0127$ & 3.0 \\
\hline G35.793-00.175 & 1 & $\mathrm{~J} 1907+0127$ & 2.7 \\
\hline \multirow[t]{2}{*}{ G36.115+00.552 } & 1 & $\mathrm{~J} 1907+0127$ & 3.4 \\
\hline & $4 c$ & $\mathrm{~J} 1907+0127$ & 3.0 \\
\hline G36.705+00.096 & $4 \mathrm{c}$ & $\mathrm{J} 1907+0127$ & 3.1 \\
\hline G37.030-00.039 & $3 b$ & $\mathrm{~J} 1856+0610$ & 2.6 \\
\hline G37.479-00.105 & $3 b$ & $\mathrm{~J} 1856+0610$ & 2.4 \\
\hline G37.598+00.425 & $3 b$ & $\mathrm{~J} 1856+0610$ & 1.9 \\
\hline G38.038-00.300 & $3 b$ & $\mathrm{~J} 1856+0610$ & 2.2 \\
\hline G38.203-00.067 & $3 b$ & $\mathrm{~J} 1856+0610$ & 2.0 \\
\hline G39.100+00.491 & $3 b$ & $\mathrm{~J} 1856+0610$ & 1.2 \\
\hline
\end{tabular}

${ }^{1}$ Source from Walsh et al. (1998).

${ }^{2}$ Sources from Beuther et al. (2002). 
A. Bartkiewicz et al.: The diversity of methanol maser morphologies from VLBI, Online Material p 3

Table 3. Targets not detected with the MERLIN single baseline. The source names, coordinates, and peak flux velocities are taken from Szymczak et al. (2002).

\begin{tabular}{ccc}
\hline \hline $\begin{array}{c}\text { Source } \\
\text { G1l.11 } \pm \text { bb.bb }\end{array}$ & $\begin{array}{c}\text { RA, Dec. } \\
(\mathrm{J} 2000)\end{array}$ & $\begin{array}{c}V_{\mathrm{p}} \\
\left(\mathrm{km} \mathrm{s}^{-1}\right)\end{array}$ \\
\hline G21.57-00.03 & $183036.5,-100643$ & +117 \\
$\mathrm{G} 22.05+00.22$ & $183035.7,-093426$ & +54 \\
$\mathrm{G} 24.93+00.08$ & $183629.2,-070505$ & +53 \\
$\mathrm{G} 26.65+00.02$ & $183951.8,-053452$ & +107 \\
$\mathrm{G} 27.21+00.26$ & $184003.8,-045809$ & +9 \\
$\mathrm{G} 27.78+00.07$ & $184147.5,-043311$ & +112 \\
$\mathrm{G} 28.02-00.44$ & $184402.1,-043414$ & +17 \\
$\mathrm{G} 28.40+00.07$ & $184254.5,-040004$ & +69 \\
$\mathrm{G} 28.53+00.12$ & $184257.7,-035159$ & +25 \\
G28.69+00.41 & $184213.6,-033507$ & +94 \\
G28.85+00.50 & $184212.8,-032426$ & +83 \\
G29.31-00.15 & $184523.1,-031723$ & +48 \\
G33.74-00.15 & $185326.9,+003901$ & +54 \\
G33.86+00.01 & $185305.2,+004936$ & +67 \\
G34.10+00.01 & $185331.9,+010226$ & +56 \\
G37.53-00.11 & $190014.4,+040235$ & +50 \\
G38.12-00.24 & $190147.6,+043032$ & +70 \\
G38.26-00.08 & $190128.7,+044202$ & +16 \\
\hline
\end{tabular}


A. Bartkiewicz et al.: The diversity of methanol maser morphologies from VLBI, Online Material $p 4$

Table 6. Maser clusters towards 31 sources. If a spectrum of a maser cluster does not show a Gaussian profile we enter the sign - in the Cols. of $F W H M$ and $S_{\text {amp. }}$.

\begin{tabular}{|c|c|c|c|c|c|}
\hline $\begin{array}{r}V_{\mathrm{LSR}} \\
\left(\mathrm{km} \mathrm{s}^{-1}\right) \\
\end{array}$ & $\begin{array}{r}\Delta \mathrm{RA} \\
\text { (mas) } \\
\end{array}$ & $\begin{array}{r}\text { Dec. } \\
(\mathrm{mas})\end{array}$ & $\begin{array}{c}S_{\mathrm{p}} \\
\left(\mathrm{mJy}^{-1} \text { beam }^{-1}\right)\end{array}$ & $\begin{array}{l}F W H M \\
\left(\mathrm{~km} \mathrm{~s}^{-1}\right)\end{array}$ & $\begin{array}{c}S_{\mathrm{amp}} \\
\left(\mathrm{mJy} \mathrm{beam}^{-1}\right)\end{array}$ \\
\hline \multicolumn{6}{|c|}{ G21.407-00.254 } \\
\hline 89.040 & 0.0000 & 0.0000 & 2762 & 0.32 & 2672 \\
\hline 89.303 & -0.0192 & 9.7250 & 1426 & 0.35 & 1513 \\
\hline 90.094 & -8.9947 & -26.3260 & 307 & 0.18 & 308 \\
\hline 91.148 & -136.3023 & 5.0420 & 891 & 0.26 & 888 \\
\hline 91.412 & -13.1560 & -23.6830 & 117 & - & - \\
\hline 91.587 & -27.3535 & 1.5320 & 422 & 0.26 & 420 \\
\hline \multicolumn{6}{|c|}{ G22.335-00.155 } \\
\hline 35.626 & 0.0000 & 0.0000 & 1714 & 0.32 & 1642 \\
\hline 38.174 & -10.9782 & -47.4060 & 125 & 0.53 & 125 \\
\hline \multicolumn{6}{|c|}{ G22.357+00.066 } \\
\hline 79.708 & 0.0000 & 0.0000 & 10544 & 0.37 & 11350 \\
\hline 80.235 & 1.0074 & -6.0100 & 10067 & 0.47 & 10190 \\
\hline 81.114 & 2.8964 & -18.3490 & 1422 & 0.48 & 1497 \\
\hline 81.465 & 155.0868 & -46.3230 & 492 & - & - \\
\hline 83.574 & 139.2789 & -18.6790 & 309 & - & - \\
\hline 84.101 & 141.5278 & 66.5690 & 156 & 0.58 & 158 \\
\hline 84.804 & 132.0327 & 75.7690 & 176 & - & - \\
\hline 88.143 & 55.7575 & 208.5390 & 602 & 0.27 & 636 \\
\hline 88.494 & 68.1772 & 277.2010 & 250 & - & - \\
\hline 88.670 & 56.6464 & 207.5440 & 476 & - & - \\
\hline \multicolumn{6}{|c|}{ G23.207-00.377 } \\
\hline 72.553 & -101.3966 & -64.2970 & 141 & 0.31 & 140 \\
\hline 73.959 & -32.5775 & -17.5310 & 194 & 0.28 & 190 \\
\hline 75.190 & 0.5751 & 9.9430 & 226 & 0.23 & 223 \\
\hline 75.014 & -25.7012 & -1.9800 & 421 & 0.83 & 374 \\
\hline 75.453 & -269.6437 & 26.1920 & 893 & 0.34 & 946 \\
\hline 75.629 & 57.3581 & 41.8210 & 647 & 0.34 & 683 \\
\hline 76.069 & -30.8269 & -11.6140 & 229 & 0.42 & 228 \\
\hline 76.596 & -11.6581 & 3.1990 & 4748 & 0.43 & 4761 \\
\hline 77.123 & 0.0000 & 0.0000 & 9292 & 0.34 & 9468 \\
\hline 77.475 & 29.4751 & -4.2880 & 3387 & 0.46 & 3463 \\
\hline 77.739 & 11.0845 & 4.9910 & 3096 & 0.47 & 2852 \\
\hline 78.793 & 53.0729 & -33.4270 & 541 & 0.44 & 563 \\
\hline 78.881 & -217.1237 & 30.9130 & 286 & 0.28 & 293 \\
\hline 78.969 & 47.4388 & -30.9230 & 577 & - & - \\
\hline 79.233 & 50.4581 & -32.3460 & 1319 & 0.38 & 1412 \\
\hline 79.496 & -135.9039 & -191.481 & 0277 & 0.21 & 294 \\
\hline 79.672 & -113.8403 & -117.084 & 0213 & 0.41 & 221 \\
\hline 79.936 & -57.8547 & -71.4830 & 315 & 0.29 & 303 \\
\hline 80.727 & -163.9145 & -147.143 & 07092 & 0.36 & 6989 \\
\hline 81.869 & -136.2582 & -139.260 & 08967 & 0.37 & 8838 \\
\hline 81.869 & -141.0800 & -140.772 & 05874 & 0.60 & 5918 \\
\hline 82.045 & -157.2072 & -145.562 & 01104 & 0.25 & 1119 \\
\hline 82.836 & -151.9599 & -147.620 & 01190 & 0.43 & 1012 \\
\hline 84.858 & -47.3987 & -112.077 & 0791 & 0.56 & 679 \\
\hline \multicolumn{6}{|c|}{ G23.389+00.185 } \\
\hline 72.641 & -78.2268 & 32.6440 & 6398 & 0.46 & 6279 \\
\hline 73.784 & 52.4158 & 32.4260 & 9326 & 0.47 & 8831 \\
\hline 74.399 & 46.8761 & 38.8880 & 2978 & 0.41 & 2715 \\
\hline 74.575 & -43.2789 & 31.9400 & 4700 & 0.28 & 4730 \\
\hline 74.750 & 43.4540 & 63.9750 & 311 & - & - \\
\hline 74.750 & 41.0708 & 14.0150 & 267 & - & - \\
\hline 74.838 & 31.5347 & 70.8960 & 19723 & 0.71 & 21320 \\
\hline 74.926 & 41.8691 & 49.9560 & 1272 & 0.17 & 1232 \\
\hline 75.453 & 0.0000 & 0.0000 & 21554 & 0.26 & 21587 \\
\hline 75.541 & -57.2121 & 153.9020 & 397 & - & - \\
\hline
\end{tabular}


A. Bartkiewicz et al.: The diversity of methanol maser morphologies from VLBI, Online Material p 5

Table 6. continued.

\begin{tabular}{|c|c|c|c|c|c|}
\hline $\begin{array}{r}V_{\mathrm{LSR}} \\
\left(\mathrm{km} \mathrm{s}^{-1}\right) \\
\end{array}$ & $\begin{array}{r}\Delta \mathrm{RA} \\
\text { (mas) } \\
\end{array}$ & $\begin{array}{r}\Delta \text { Dec. } \\
\text { (mas) }\end{array}$ & $\begin{array}{c}S_{\mathrm{p}} \\
\left(\mathrm{mJy}^{-1} \text { beam }^{-1}\right)\end{array}$ & $\begin{array}{l}F W H M \\
\left(\mathrm{~km} \mathrm{~s}^{-1}\right)\end{array}$ & $\begin{array}{c}S_{\mathrm{amp}} \\
\left(\mathrm{mJy} \mathrm{beam}^{-1}\right)\end{array}$ \\
\hline 75.805 & -64.5014 & 143.6490 & 3708 & 0.32 & 3768 \\
\hline 76.069 & 78.5948 & 144.9390 & 6571 & 0.37 & 6686 \\
\hline 76.332 & -93.2091 & 154.8360 & 4516 & 0.62 & 4683 \\
\hline 76.596 & -70.0292 & 145.2360 & 1072 & 0.55 & 1086 \\
\hline 76.948 & 20.9791 & 75.4450 & 721 & 0.31 & 727 \\
\hline 77.035 & -69.1893 & 147.1100 & 4860 & 0.28 & 4795 \\
\hline 77.475 & -73.8030 & 150.8570 & 234 & 0.19 & 234 \\
\hline 77.563 & -31.8834 & 157.9720 & 485 & 0.24 & 470 \\
\hline \multicolumn{6}{|c|}{ G23.657-00.127 } \\
\hline 77.563 & -5.0421 & 229.8720 & 816 & 0.42 & 754 \\
\hline 77.563 & -22.2581 & 232.7000 & 333 & 0.25 & 355 \\
\hline 78.266 & -71.1275 & 228.1640 & 1059 & 0.52 & 932 \\
\hline 78.881 & 54.5928 & 171.6190 & 336 & 0.50 & 305 \\
\hline 79.584 & 105.3428 & 117.0720 & 162 & 0.75 & 170 \\
\hline 80.024 & -124.7437 & 212.4980 & 1595 & 0.23 & 1607 \\
\hline 80.112 & -39.3568 & 213.5540 & 977 & 0.32 & 938 \\
\hline 80.639 & 21.7712 & -13.2960 & 850 & 0.49 & 691 \\
\hline 80.727 & 14.3113 & -66.4860 & 375 & 0.20 & 391 \\
\hline 80.990 & -156.9775 & -5.2880 & 124 & 0.25 & 135 \\
\hline 81.166 & 2.9967 & -47.4670 & 362 & 0.30 & 370 \\
\hline 81.166 & 26.3472 & -70.2780 & 68 & 0.55 & 77 \\
\hline 81.518 & 7.2432 & -3.7020 & 588 & 0.42 & 564 \\
\hline 81.781 & 46.3136 & 156.5250 & 126 & 0.39 & 117 \\
\hline 81.869 & 10.2711 & 10.8770 & 806 & 0.30 & 820 \\
\hline 81.869 & 13.2471 & -13.0970 & 90 & 0.19 & 92 \\
\hline 82.133 & -1.5481 & -43.7250 & 132 & 0.31 & 124 \\
\hline 82.309 & 44.5072 & 156.8130 & 393 & 0.28 & 391 \\
\hline 82.573 & 0.0000 & 0.0000 & 3623 & 0.48 & 3401 \\
\hline 82.836 & 41.5298 & 162.2880 & 168 & 0.29 & 174 \\
\hline 83.100 & -210.9484 & 11.3450 & 182 & 0.24 & 196 \\
\hline 83.276 & -47.8646 & -35.5030 & 1018 & 0.33 & 1036 \\
\hline 83.539 & 69.2069 & 83.7510 & 80 & 0.27 & 81 \\
\hline 83.979 & 0.7674 & 18.7780 & 143 & 0.62 & 125 \\
\hline 83.979 & -52.8072 & -32.5950 & 557 & 0.58 & 531 \\
\hline 84.243 & -133.7606 & -4.1400 & 358 & 0.27 & 373 \\
\hline 84.858 & 45.6887 & 140.0160 & 548 & 0.44 & 552 \\
\hline 86.264 & -17.8008 & -15.6640 & 797 & 0.37 & 766 \\
\hline 86.791 & -19.7200 & -18.7010 & 746 & 0.37 & 702 \\
\hline 87.231 & -16.7410 & -26.9680 & 96 & 0.24 & 96 \\
\hline 87.670 & -20.3523 & -29.5260 & 280 & 0.26 & 272 \\
\hline \multicolumn{6}{|c|}{ G23.707-00.198 } \\
\hline 58.578 & 20.4283 & 18.1540 & 377 & - & \\
\hline 71.410 & -101.2879 & 0.5490 & 200 & 0.19 & 207 \\
\hline 71.762 & -105.4053 & -5.6330 & 128 & 0.19 & 141 \\
\hline 72.993 & -10.2000 & 57.1700 & 667 & 0.23 & 707 \\
\hline 73.344 & -1.6921 & 33.7300 & 92 & 0.40 & 95 \\
\hline 73.608 & 0.3488 & 27.4820 & 100 & 0.22 & 102 \\
\hline 74.223 & -3.2461 & 38.1980 & 672 & 0.38 & 683 \\
\hline 74.575 & 0.3933 & 23.6850 & 1845 & 0.26 & 1756 \\
\hline 75.102 & -0.0089 & 29.0830 & 404 & 0.47 & 416 \\
\hline 75.190 & 16.6285 & -9.9600 & 306 & 0.52 & 340 \\
\hline 75.629 & 0.3577 & 26.8210 & 249 & 0.50 & 260 \\
\hline 76.157 & 0.9722 & 19.8400 & 3319 & 0.46 & 3297 \\
\hline 76.596 & 0.9589 & 17.6580 & 2054 & 0.83 & 2033 \\
\hline 77.299 & 0.8772 & 16.2390 & 3473 & 0.29 & 3625 \\
\hline 78.266 & -14.6856 & -14.3980 & 219 & 0.31 & 219 \\
\hline 78.530 & -13.7935 & -14.4180 & 358 & 0.32 & 366 \\
\hline
\end{tabular}


A. Bartkiewicz et al.: The diversity of methanol maser morphologies from VLBI, Online Material p 6

Table 6. continued.

\begin{tabular}{|c|c|c|c|c|c|}
\hline $\begin{array}{r}V_{\text {LSR }} \\
\left(\mathrm{km} \mathrm{s}^{-1}\right)\end{array}$ & $\begin{array}{r}\Delta \mathrm{RA} \\
(\mathrm{mas})\end{array}$ & $\begin{array}{l}\text { Dec. } \\
\text { (mas) }\end{array}$ & $\begin{array}{c}S_{\mathrm{p}} \\
\left(\text { mJy beam }^{-1}\right)\end{array}$ & $\begin{array}{l}F W H M \\
\left(\mathrm{~km} \mathrm{~s}^{-1}\right)\end{array}$ & $\begin{array}{c}S_{\mathrm{amp}} \\
\left(\mathrm{mJy} \mathrm{beam}^{-1}\right)\end{array}$ \\
\hline 77.651 & -0.4334 & 3.7530 & 2234 & 0.70 & 2239 \\
\hline 78.090 & -0.5982 & 1.0340 & 2144 & 0.95 & 2099 \\
\hline 79.145 & 0.0000 & 0.0000 & 6059 & 0.79 & 5368 \\
\hline 79.496 & -17.3246 & -13.9080 & 117 & 0.61 & 109 \\
\hline 80.112 & -14.3234 & -14.3790 & 202 & 0.36 & 200 \\
\hline 80.903 & -4.2421 & -8.0300 & 142 & 0.48 & 129 \\
\hline 81.078 & -3.9200 & -8.6280 & 159 & 0.56 & 153 \\
\hline \multicolumn{6}{|c|}{ G23.966-00.109 } \\
\hline 67.428 & 21.9498 & -23.0050 & 387 & 0.30 & 369 \\
\hline 68.219 & 23.3000 & -22.9600 & 431 & 0.41 & 404 \\
\hline 70.942 & 0.0000 & 0.0000 & 5487 & 0.41 & 5390 \\
\hline \multicolumn{6}{|c|}{ G24.148-00.009 } \\
\hline 17.441 & 4.3548 & -21.7190 & 1048 & 0.20 & 1048 \\
\hline 17.529 & 0.1980 & -0.0970 & 2459 & 0.51 & 2493 \\
\hline 17.792 & 0.0000 & 0.0000 & 3648 & 0.40 & 3702 \\
\hline 18.407 & 0.6149 & 5.9860 & 240 & 0.33 & 235 \\
\hline \multicolumn{6}{|c|}{$\mathrm{G} 24.541+00.312$} \\
\hline 103.754 & -35.7935 & 59.5820 & 233 & 0.23 & 233 \\
\hline 105.688 & 0.0000 & 0.0000 & 7753 & 0.34 & 7453 \\
\hline 106.215 & -38.6463 & 37.3600 & 316 & 0.29 & 278 \\
\hline 107.973 & -10.8681 & 7.8090 & 238 & 0.33 & 239 \\
\hline 106.479 & -5.5553 & 2.9680 & 2788 & 0.49 & 2554 \\
\hline 107.270 & 0.0253 & 1.0010 & 2700 & 0.47 & 2677 \\
\hline 107.533 & -1.8840 & 2.4950 & 2005 & 0.38 & 2052 \\
\hline 108.324 & 94.8606 & 14.3270 & 106 & 0.32 & 108 \\
\hline 108.588 & -26.0490 & 13.4890 & 638 & 0.37 & 534 \\
\hline 109.467 & -39.7491 & 16.3690 & 339 & 0.57 & 339 \\
\hline 109.643 & -39.9396 & 16.9440 & 407 & 0.38 & 413 \\
\hline 110.082 & -41.4218 & 18.0810 & 728 & 0.39 & 742 \\
\hline \multicolumn{6}{|c|}{ G24.634-00.324 } \\
\hline 34.725 & -18.6698 & 25.4370 & 457 & - & - \\
\hline 35.428 & 0.0000 & 0.0000 & 3027 & 0.29 & 3544 \\
\hline 43.862 & -62.2684 & 36.4860 & 2370 & 0.56 & 2322 \\
\hline 45.795 & -41.9147 & 45.7150 & 257 & 0.41 & 257 \\
\hline 46.322 & -43.8591 & 45.1230 & 312 & 0.38 & 313 \\
\hline 47.904 & -42.4775 & 45.4230 & 136 & 0.54 & 136 \\
\hline \multicolumn{6}{|c|}{ G25.411+00.105 } \\
\hline 93.765 & 34.1096 & 111.2790 & 142 & - & - \\
\hline 94.117 & 109.0956 & -19.4400 & 82 & - & - \\
\hline 94.820 & 115.8608 & 101.1940 & 847 & - & - \\
\hline 94.644 & -16.7363 & 86.3980 & 2374 & 0.51 & 2345 \\
\hline 94.820 & -3.5683 & -20.6650 & 1025 & 0.50 & 1037 \\
\hline 96.928 & -3.5146 & 123.8050 & 323 & - & - \\
\hline 96.928 & 3.2029 & -5.4840 & 2251 & 0.42 & 2241 \\
\hline 97.104 & -46.6480 & 16.7050 & 2468 & 0.28 & 2504 \\
\hline 97.280 & 0.0000 & 0.0000 & 3433 & 0.56 & 3690 \\
\hline 98.861 & 176.2362 & 50.0100 & 139 & - & - \\
\hline \multicolumn{6}{|c|}{ G26.598-00.024 } \\
\hline 22.952 & 2.1488 & -0.1130 & 182 & 0.45 & 183 \\
\hline 24.182 & 0.0000 & 0.0000 & 3043 & 0.34 & 3239 \\
\hline 24.709 & 84.5157 & -169.5700 & 1368 & 0.44 & 1276 \\
\hline 25.061 & 335.4955 & -131.1280 & 714 & 0.51 & 671 \\
\hline 25.588 & 78.8583 & -159.7380 & 76 & - & - \\
\hline 25.939 & 338.8875 & -124.8040 & 97 & 0.94 & 99 \\
\hline
\end{tabular}


A. Bartkiewicz et al.: The diversity of methanol maser morphologies from VLBI, Online Material $p 7$

Table 6. continued.

\begin{tabular}{|c|c|c|c|c|c|}
\hline $\begin{array}{r}V_{\text {LSR }} \\
\left(\mathrm{km} \mathrm{s}^{-1}\right)\end{array}$ & $\begin{array}{r}\Delta \mathrm{RA} \\
(\mathrm{mas})\end{array}$ & $\begin{array}{r}\Delta \text { Dec. } \\
\text { (mas) }\end{array}$ & $\begin{array}{c}S_{\mathrm{p}} \\
\left(\text { mJy beam }^{-1}\right)\end{array}$ & $\begin{array}{c}F W H M \\
\left(\mathrm{~km} \mathrm{~s}^{-1}\right)\end{array}$ & $\begin{array}{c}S_{\text {amp }} \\
\left(\text { mJy beam }^{-1}\right)\end{array}$ \\
\hline \multicolumn{6}{|c|}{ G27.221+00.136 } \\
\hline 105.424 & -11.6570 & 77.8590 & 253 & 0.42 & 237 \\
\hline 106.391 & -11.2849 & 77.8360 & 94 & 0.59 & 91 \\
\hline 107.182 & -33.2614 & 55.4880 & 245 & 0.42 & 232 \\
\hline 109.731 & -33.8577 & 32.9640 & 1239 & 0.26 & 1193 \\
\hline 110.258 & -34.2955 & 34.2190 & 1182 & 0.43 & 1179 \\
\hline 111.225 & -33.0149 & 35.1860 & 467 & 0.30 & 466 \\
\hline 112.280 & -19.4363 & 27.0680 & 563 & 0.35 & 538 \\
\hline 112.719 & -18.6697 & 27.1140 & 906 & 0.29 & 917 \\
\hline 114.828 & -20.1700 & 27.8700 & 166 & 0.25 & 169 \\
\hline 115.268 & -18.4171 & 23.1670 & 150 & 0.39 & 152 \\
\hline 115.444 & -19.0119 & 24.4340 & 158 & - & - \\
\hline 115.707 & -24.1941 & 47.1650 & 236 & 0.31 & 225 \\
\hline 116.586 & -24.7798 & 7.8580 & 437 & 0.23 & 457 \\
\hline 117.114 & -53.1908 & 8.6200 & 6519 & 0.33 & 6591 \\
\hline 117.729 & 8.9344 & -12.1430 & 448 & 0.23 & 469 \\
\hline 117.817 & -54.3145 & 7.6670 & 5642 & 0.55 & 5621 \\
\hline 118.168 & -9.3558 & -11.4900 & 1991 & 0.33 & 1990 \\
\hline 118.432 & -55.3082 & 7.0300 & 4831 & 0.35 & 4755 \\
\hline 118.783 & 0.0000 & 0.0000 & 12535 & 0.39 & 12021 \\
\hline 118.959 & -8.6878 & 13.1750 & 765 & 0.67 & 801 \\
\hline 119.223 & -70.7368 & -6.8450 & 209 & - & - \\
\hline 119.311 & -9.2317 & -5.7490 & 232 & 0.33 & 224 \\
\hline 119.311 & -34.3642 & 4.9240 & 135 & 0.51 & 111 \\
\hline 119.750 & -9.4066 & 11.3470 & 101 & 0.38 & 103 \\
\hline 120.365 & -15.4465 & -18.4780 & 536 & 0.23 & 536 \\
\hline 120.190 & -20.1998 & 41.8670 & 1339 & 0.40 & 1367 \\
\hline 121.069 & -17.4294 & 44.0510 & 218 & 0.29 & 218 \\
\hline \multicolumn{6}{|c|}{$\mathrm{G} 28.817+00.365$} \\
\hline 87.735 & -73.2105 & -56.0600 & 325 & 0.51 & 331 \\
\hline 88.087 & -63.9892 & -72.9580 & 183 & - & - \\
\hline 90.723 & 0.0000 & 0.0000 & 3137 & 0.36 & 3211 \\
\hline 91.074 & 2.8641 & 17.4230 & 1440 & 0.62 & 1440 \\
\hline 91.250 & 2.7787 & 3.5790 & 896 & 0.68 & 933 \\
\hline 91.601 & 4.0115 & 20.0900 & 2511 & 0.31 & 2755 \\
\hline 92.129 & -3.1337 & 10.3800 & 743 & 0.47 & 761 \\
\hline 92.656 & -2.7143 & 26.7830 & 2116 & 0.38 & 2103 \\
\hline \multicolumn{6}{|c|}{ G30.318+00.070 } \\
\hline 35.192 & -23.8385 & 22.6390 & 300 & 0.53 & 303 \\
\hline 36.070 & 0.0000 & 0.0000 & 514 & 0.68 & 528 \\
\hline 36.949 & 5.6770 & -5.0420 & 144 & 0.70 & 144 \\
\hline \multicolumn{6}{|c|}{ G30.400-00.296 } \\
\hline 98.104 & 4.4898 & -11.8250 & 2139 & 0.31 & 2151 \\
\hline 98.455 & 0.0000 & 0.0000 & 2765 & 0.32 & 3129 \\
\hline 100.388 & -31.0281 & -45.2180 & 1581 & 0.29 & 1603 \\
\hline 101.618 & -27.7091 & 85.7960 & 331 & 0.72 & 326 \\
\hline 103.727 & -19.1733 & 86.2030 & 1914 & 0.41 & 1923 \\
\hline 103.727 & 67.4799 & 134.7790 & 497 & 0.28 & 503 \\
\hline 104.430 & 66.4200 & 135.2860 & 260 & 0.43 & 267 \\
\hline
\end{tabular}


A. Bartkiewicz et al.: The diversity of methanol maser morphologies from VLBI, Online Material $p 8$

Table 6. continued.

\begin{tabular}{|c|c|c|c|c|c|}
\hline $\begin{array}{r}V_{\text {LSR }} \\
\left(\mathrm{km} \mathrm{s}^{-1}\right) \\
\end{array}$ & $\begin{array}{r}\mathrm{RA} \\
(\mathrm{mas}) \\
\end{array}$ & $\begin{array}{r}\Delta \text { Dec. } \\
(\text { mas })\end{array}$ & $\begin{array}{c}S_{\mathrm{p}} \\
\left(\mathrm{mJy}^{-1} \text { beam }^{-1}\right)\end{array}$ & $\begin{array}{l}F W H M \\
\left(\mathrm{~km} \mathrm{~s}^{-1}\right) \\
\end{array}$ & $\begin{array}{c}S_{\mathrm{amp}} \\
\left(\mathrm{mJy} \mathrm{beam}^{-1}\right)\end{array}$ \\
\hline \multicolumn{6}{|c|}{ G31.047+00.356 } \\
\hline 81.058 & -59.6159 & -28.5990 & 277 & - & - \\
\hline 78.070 & -0.9674 & 7.7850 & 281 & 0.82 & 267 \\
\hline 79.125 & -1.2973 & 3.2920 & 1169 & 0.73 & 1180 \\
\hline 80.179 & 0.4664 & 1.5320 & 544 & 0.55 & 555 \\
\hline 80.882 & 0.0000 & 0.0000 & 1989 & 0.55 & 1960 \\
\hline 81.058 & -0.3989 & -1.5250 & 1563 & 0.55 & 1568 \\
\hline 82.991 & -47.8577 & -6.9740 & 617 & 0.28 & 651 \\
\hline 84.045 & -25.8591 & 9.6530 & 101 & - & - \\
\hline \multicolumn{6}{|c|}{ G31.581+00.077 } \\
\hline 95.643 & 0.0000 & 0.0000 & 2722 & 0.35 & 2891 \\
\hline 97.752 & -60.9387 & 57.6080 & 349 & - & - \\
\hline 98.104 & 37.3458 & -7.0480 & 809 & 0.31 & 814 \\
\hline 98.455 & 32.2616 & -3.7570 & 376 & 0.39 & 386 \\
\hline 98.631 & 149.7072 & 94.0260 & 493 & - & - \\
\hline 98.806 & -61.9691 & 79.9160 & 292 & 0.41 & 321 \\
\hline 98.806 & 149.8767 & 93.2150 & 2045 & 0.68 & 2039 \\
\hline 99.509 & 158.1329 & 69.4150 & 358 & - & - \\
\hline 99.685 & 151.6794 & 56.5090 & 1099 & 0.39 & 1223 \\
\hline \multicolumn{6}{|c|}{ G32.992+00.034 } \\
\hline 89.724 & 13.3545 & -44.5810 & 321 & - & - \\
\hline 90.339 & -51.5775 & 49.8690 & 396 & - & - \\
\hline 90.602 & 14.7555 & -34.4810 & 1967 & 0.40 & 1953 \\
\hline 90.954 & 26.7960 & 1.1600 & 4650 & 0.32 & 4838 \\
\hline 91.393 & -4.7370 & -6.0210 & 493 & 0.50 & 505 \\
\hline 91.481 & 28.3665 & 0.9760 & 1856 & 0.50 & 1867 \\
\hline 91.656 & -16.1355 & 37.9820 & 465 & - & - \\
\hline 91.832 & 0.0000 & 0.0000 & 6212 & 0.35 & 6786 \\
\hline 92.184 & 34.7340 & -14.9630 & 394 & 0.28 & 394 \\
\hline 92.447 & 35.5635 & -14.7320 & 163 & - & - \\
\hline 92.711 & 24.9990 & -19.6750 & 199 & - & - \\
\hline 94.204 & 51.0915 & -1.6040 & 600 & 0.26 & 574 \\
\hline 94.731 & 49.8900 & -4.5590 & 445 & 0.22 & 446 \\
\hline \multicolumn{6}{|c|}{ G33.641-00.228 } \\
\hline 58.840 & 0.0000 & 0.0000 & 28300 & 0.31 & 29569 \\
\hline 59.540 & -9.5996 & -12.3000 & 4105 & 0.29 & 4259 \\
\hline 59.800 & -14.3994 & -20.1000 & 12260 & 0.22 & 12665 \\
\hline 60.330 & 69.5970 & -15.9000 & 20402 & 0.30 & 21448 \\
\hline 60.420 & 61.9473 & -25.9000 & 2280 & 0.50 & 2688 \\
\hline 60.510 & 60.8973 & -27.0000 & 1621 & - & - \\
\hline 60.860 & 13.3494 & -28.6000 & 1672 & 0.30 & 1716 \\
\hline 60.940 & 65.3971 & -14.6000 & 10447 & 0.35 & 10558 \\
\hline 61.120 & 76.3467 & -2.3000 & 356 & 0.35 & 372 \\
\hline 61.300 & 56.2475 & -17.0000 & 207 & 0.26 & 213 \\
\hline 61.300 & 128.3944 & 55.9000 & 176 & - & - \\
\hline 61.820 & 82.1964 & 7.6000 & 365 & 0.40 & 358 \\
\hline 62.260 & 122.5447 & 63.5000 & 893 & 0.56 & 812 \\
\hline 62.180 & 111.2951 & 50.0000 & 109 & - & - \\
\hline 62.440 & 42.5981 & -18.2000 & 5440 & 0.31 & 5758 \\
\hline 62.700 & 42.5981 & -17.8000 & 20690 & 0.35 & 20872 \\
\hline 62.970 & 42.5981 & -16.6000 & 9325 & 0.48 & 9620 \\
\hline 63.140 & 30.4487 & -19.7000 & 1090 & 0.30 & 1124 \\
\hline \multicolumn{6}{|c|}{ G33.980-00.019 } \\
\hline 58.886 & 0.0000 & 0.0000 & 3782 & 0.33 & 4057 \\
\hline 59.501 & 5.1225 & 0.1130 & 434 & 0.30 & 454 \\
\hline 60.292 & -50.1990 & -23.9650 & 315 & 0.45 & 290 \\
\hline
\end{tabular}


A. Bartkiewicz et al.: The diversity of methanol maser morphologies from VLBI, Online Material $p 9$

Table 6. continued.

\begin{tabular}{|c|c|c|c|c|c|}
\hline $\begin{array}{r}V_{\text {LSR }} \\
\left(\mathrm{km} \mathrm{s}^{-1}\right)\end{array}$ & $\begin{array}{c}\mathrm{RA} \\
\text { (mas) }\end{array}$ & $\begin{array}{l}\text { Dec. } \\
\text { (mas) }\end{array}$ & $\begin{array}{c}S_{\mathrm{p}} \\
\left(\mathrm{mJy}_{\text {beam }}^{-1}\right)\end{array}$ & $\begin{array}{l}F W H M \\
\left(\mathrm{~km} \mathrm{~s}^{-1}\right)\end{array}$ & $\begin{array}{c}S_{\mathrm{amp}} \\
\left(\mathrm{mJy} \mathrm{beam}^{-1}\right)\end{array}$ \\
\hline 60.555 & -29.6685 & 1.7760 & 254 & 0.29 & 258 \\
\hline 61.258 & -49.1925 & -16.8830 & 784 & 0.37 & 797 \\
\hline 61.609 & 30.1755 & -5.5850 & 515 & 0.60 & 469 \\
\hline 62.312 & 30.5595 & -3.2980 & 276 & 0.40 & 248 \\
\hline 64.157 & 16.2345 & -27.9140 & 497 & 0.50 & 471 \\
\hline 65.387 & -32.4450 & -40.9110 & 236 & 0.25 & 237 \\
\hline \multicolumn{6}{|c|}{ G34.751-00.093 } \\
\hline 50.627 & 28.8631 & -27.8740 & 224 & 0.59 & 215 \\
\hline 51.769 & -10.8418 & -3.5670 & 718 & 0.46 & 702 \\
\hline 52.736 & 0.0000 & 0.0000 & 1954 & 0.68 & 1817 \\
\hline 53.175 & -3.5980 & -0.9420 & 1763 & 0.44 & 1845 \\
\hline \multicolumn{6}{|c|}{ G35.793-00.175 } \\
\hline 60.060 & -2.4000 & -0.8600 & 889 & 0.27 & 952 \\
\hline 60.680 & 0.0000 & 0.0000 & 9702 & 0.62 & 9616 \\
\hline 61.300 & 1.9500 & 0.8100 & 6338 & 0.48 & 6327 \\
\hline 61.820 & 3.0000 & 1.2100 & 5960 & 0.75 & 5927 \\
\hline 62.350 & 4.3500 & 2.2100 & 1225 & 0.60 & 1347 \\
\hline \multicolumn{6}{|c|}{$\mathrm{G} 36.115+00.552$} \\
\hline 70.395 & -1072.0323 & 421.7720 & 10779 & 0.38 & 9795 \\
\hline 70.834 & -1075.7487 & 431.4520 & 254 & 0.33 & 261 \\
\hline 71.186 & -5.1454 & 0.8900 & 1066 & 0.40 & 1113 \\
\hline 71.449 & -1068.0478 & 420.0420 & 617 & 0.35 & 637 \\
\hline 71.801 & -1062.5353 & 420.6660 & 2921 & 0.28 & 2869 \\
\hline 71.977 & -24.9857 & -12.5080 & 1386 & 0.29 & 1398 \\
\hline 72.416 & -1064.1711 & 421.8460 & 2973 & 0.63 & 2681 \\
\hline 72.504 & -1168.7980 & 300.8850 & 506 & - & - \\
\hline 72.855 & -1063.7741 & 421.0850 & 11194 & 0.35 & 11100 \\
\hline 73.031 & 0.0000 & 0.0000 & 11744 & 0.49 & 11978 \\
\hline 73.119 & -39.3480 & -27.3450 & 1431 & 0.58 & 1613 \\
\hline 73.821 & -1163.3500 & 155.9080 & 1134 & 0.19 & 1149 \\
\hline 74.173 & -48.5034 & -43.3810 & 2845 & 0.39 & 3600 \\
\hline 74.436 & -43.6441 & -45.4110 & 6641 & 0.54 & 5863 \\
\hline 75.139 & -9.8909 & 12.9190 & 371 & 0.35 & 357 \\
\hline 75.579 & -46.4093 & -46.6400 & 316 & 0.36 & 302 \\
\hline 75.754 & 7.3175 & 14.7340 & 431 & 0.28 & 431 \\
\hline 76.106 & 0.1992 & 16.2260 & 3900 & 0.27 & 4007 \\
\hline 81.289 & -1122.9459 & 420.7460 & 1619 & 0.78 & 1373 \\
\hline 81.553 & -1100.4663 & 436.9820 & 447 & 0.77 & 386 \\
\hline 81.816 & -1103.1221 & 438.3080 & 513 & 0.27 & 515 \\
\hline 81.904 & -1116.3250 & 407.0410 & 1010 & 0.31 & 1103 \\
\hline 82.168 & -1113.5523 & 420.1690 & 5062 & 0.32 & 5183 \\
\hline 83.925 & -1116.4418 & 417.6580 & 1652 & 0.66 & 1816 \\
\hline \multicolumn{6}{|c|}{$\mathrm{G} 36.705+00.096$} \\
\hline 52.560 & 1.3392 & 1.1530 & 348 & 0.33 & 353 \\
\hline 53.087 & 0.0000 & 0.0000 & 7576 & 0.31 & 7608 \\
\hline 53.790 & -0.9167 & -0.1560 & 2413 & 0.48 & 2470 \\
\hline 54.405 & -1.8934 & -1.2010 & 4629 & 0.65 & 4404 \\
\hline 54.844 & -2.0102 & -9.9610 & 1309 & 0.43 & 1343 \\
\hline 55.108 & -1.5579 & -3.2250 & 420 & - & - \\
\hline 62.137 & -14.1016 & 52.0590 & 1974 & 0.45 & 1969 \\
\hline 63.015 & -0.3940 & 45.6080 & 153 & 0.47 & 154 \\
\hline \multicolumn{6}{|c|}{ G37.030-00.039 } \\
\hline 78.585 & 0.0000 & 0.0000 & 691 & 0.47 & 656 \\
\hline 78.761 & -0.4374 & 0.2050 & 224 & - & - \\
\hline
\end{tabular}


A. Bartkiewicz et al.: The diversity of methanol maser morphologies from VLBI, Online Material p 10

Table 6. continued.

\begin{tabular}{|c|c|c|c|c|c|}
\hline $\begin{array}{r}V_{\text {LSR }} \\
\left(\mathrm{km} \mathrm{s}^{-1}\right)\end{array}$ & $\begin{array}{r}\Delta \mathrm{RA} \\
\text { (mas) }\end{array}$ & $\begin{array}{r}\text { Dec. } \\
\text { (mas) }\end{array}$ & $\begin{array}{c}S_{\mathrm{p}} \\
\left(\text { mJy beam }^{-1}\right)\end{array}$ & $\begin{array}{l}F W H M \\
\left(\mathrm{~km} \mathrm{~s}^{-1}\right)\end{array}$ & $\begin{array}{c}\begin{array}{c}S_{\mathrm{amp}} \\
\left(\mathrm{mJy} \mathrm{beam}^{-1}\right)\end{array}\end{array}$ \\
\hline \multicolumn{6}{|c|}{ G37.598+00.425 } \\
\hline 82.802 & 91.6302 & -11.1510 & 251 & - & - \\
\hline 85.789 & 0.0000 & 0.0000 & 3911 & 0.43 & 3826 \\
\hline 85.438 & -0.3322 & -0.8860 & 891 & 0.33 & 902 \\
\hline 86.931 & 26.0379 & 12.9580 & 2407 & 0.32 & 2482 \\
\hline 87.195 & 25.4768 & 13.1770 & 465 & - & - \\
\hline 87.283 & 39.3823 & 6.436 & 2543 & 0.45 & 2661 \\
\hline 88.600 & 63.5139 & 4.0650 & 395 & - & - \\
\hline 88.688 & 66.2298 & -7.5300 & 202 & - & - \\
\hline \multicolumn{6}{|c|}{ G38.038-00.300 } \\
\hline 55.656 & 0.0000 & 0.0000 & 2168 & 0.34 & 1996 \\
\hline 57.062 & 6.2921 & -15.5470 & 507 & - & - \\
\hline 57.413 & 9.3731 & -16.4000 & 1046 & 0.38 & 980 \\
\hline 58.204 & 13.9385 & -1.8140 & 1434 & 0.25 & 1444 \\
\hline 59.522 & 20.8441 & -15.0610 & 215 & - & - \\
\hline 59.522 & 19.9328 & 12.8000 & 224 & - & - \\
\hline 59.609 & 19.0545 & -3.8880 & 281 & - & - \\
\hline \multicolumn{6}{|c|}{ G38.203-00.067 } \\
\hline 79.640 & 0.0000 & 0.0000 & 828 & 0.28 & 707 \\
\hline 80.518 & -54.8141 & 20.5190 & 169 & - & - \\
\hline 80.606 & 11.2600 & 13.4970 & 157 & 0.38 & 160 \\
\hline 83.856 & -101.8398 & 154.2200 & 173 & - & - \\
\hline 83.944 & -35.3736 & 29.9400 & 152 & - & - \\
\hline 83.944 & -35.2315 & 44.9650 & 195 & - & - \\
\hline 84.208 & -33.8743 & 31.6240 & 198 & - & - \\
\hline \multicolumn{6}{|c|}{ G39.100+00.491 } \\
\hline 14.630 & -5.7037 & -38.5700 & 222 & - & - \\
\hline 14.630 & -7.8346 & -11.4310 & 453 & 0.28 & 446 \\
\hline 15.772 & 13.3126 & -22.0650 & 314 & - & - \\
\hline 15.860 & 115.0769 & 103.8770 & 623 & - & - \\
\hline 15.860 & 116.6086 & 75.8880 & 545 & - & - \\
\hline 15.860 & 13.3112 & -22.0040 & 769 & - & - \\
\hline 15.245 & 0.0000 & 0.0000 & 2068 & 0.37 & 1803 \\
\hline 15.684 & -5.1254 & -5.8070 & 1140 & 0.62 & 1158 \\
\hline 15.948 & -4.9192 & -7.1320 & 1042 & 0.23 & 1108 \\
\hline 17.617 & 6.6630 & -8.1800 & 696 & 0.32 & 654 \\
\hline
\end{tabular}


A. Bartkiewicz et al.: The diversity of methanol maser morphologies from VLBI, Online Material p 11
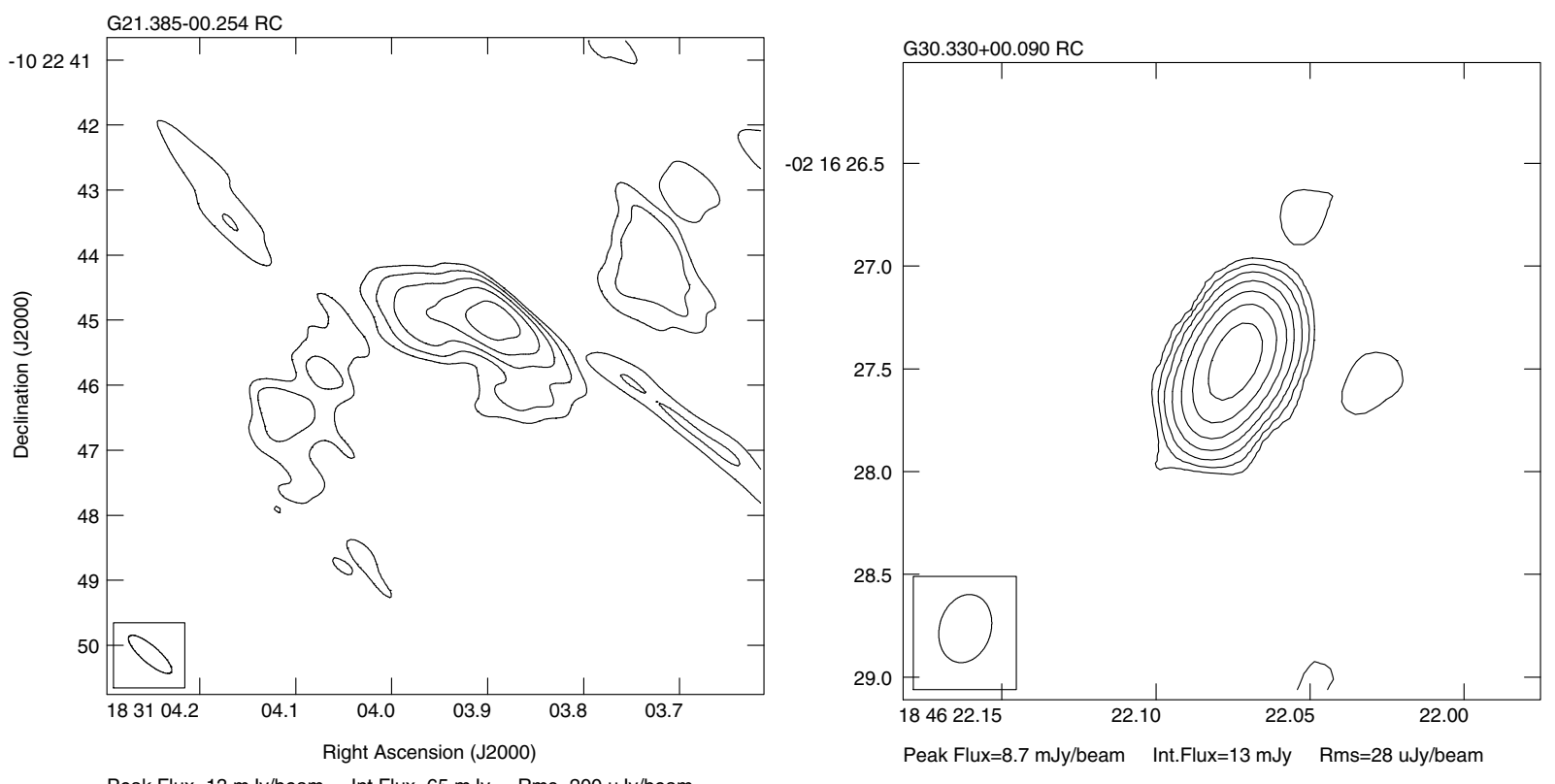

Peak Flux=13 mJy/beam Int.Flux=65 mJy Rms=200 uJy/beam
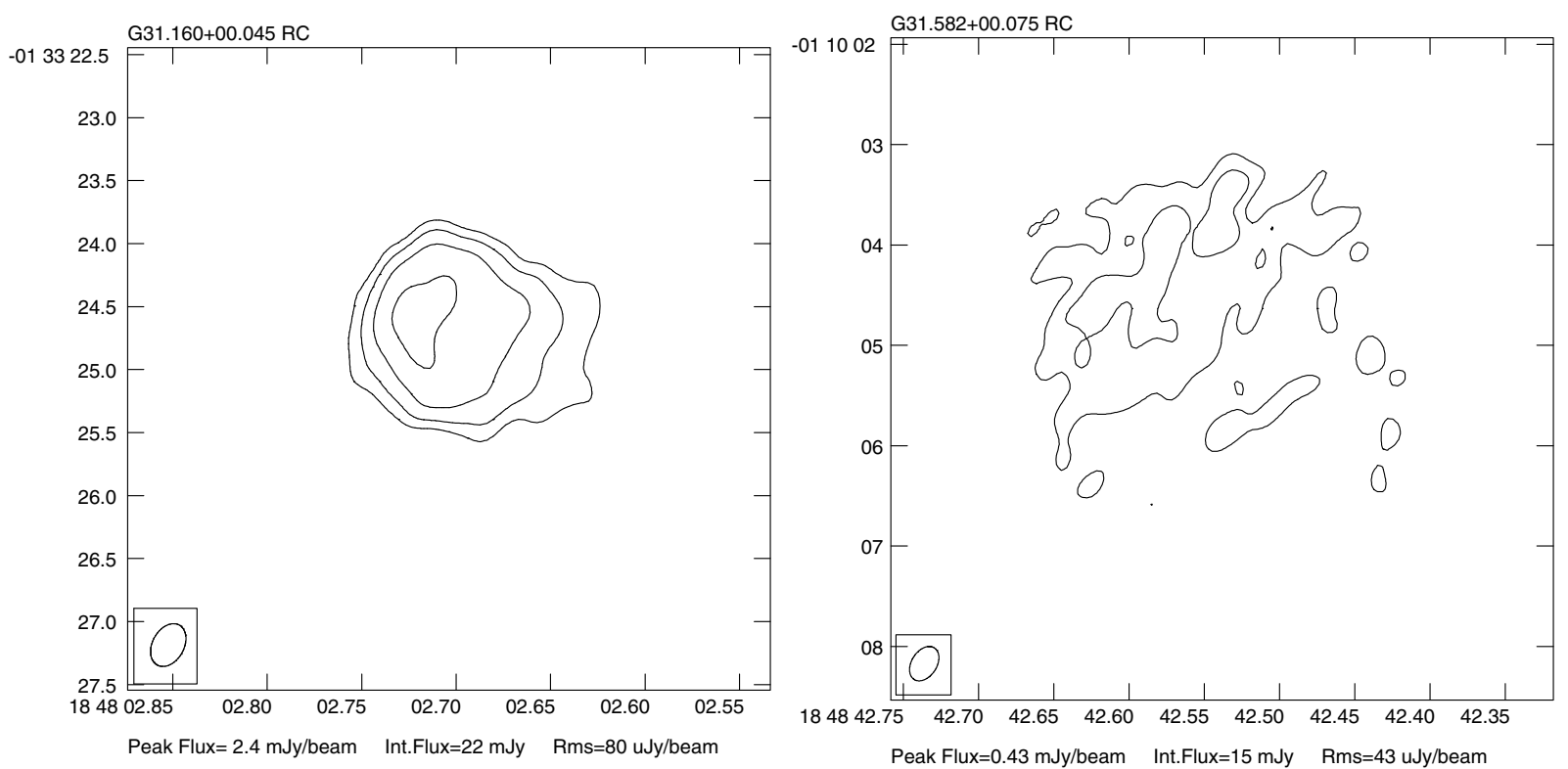

Fig. 3. The $8.4 \mathrm{GHz}$ continuum emission detected using VLA on 2007 August 18. The names of radio continuum sources (RC) are the Galactic coordinates of peak fluxes. The peak and integrated fluxes as well as the levels of rms $\left(1 \sigma_{\mathrm{rms}}\right)$ are given under each map and the beamsizes are presented at the left down corners. Contours trace the levels of $3 \sigma_{\text {rms }} \times(1,2,4,8,16,32,64, \ldots)$. 
A. Bartkiewicz et al.: The diversity of methanol maser morphologies from VLBI, Online Material $p 12$
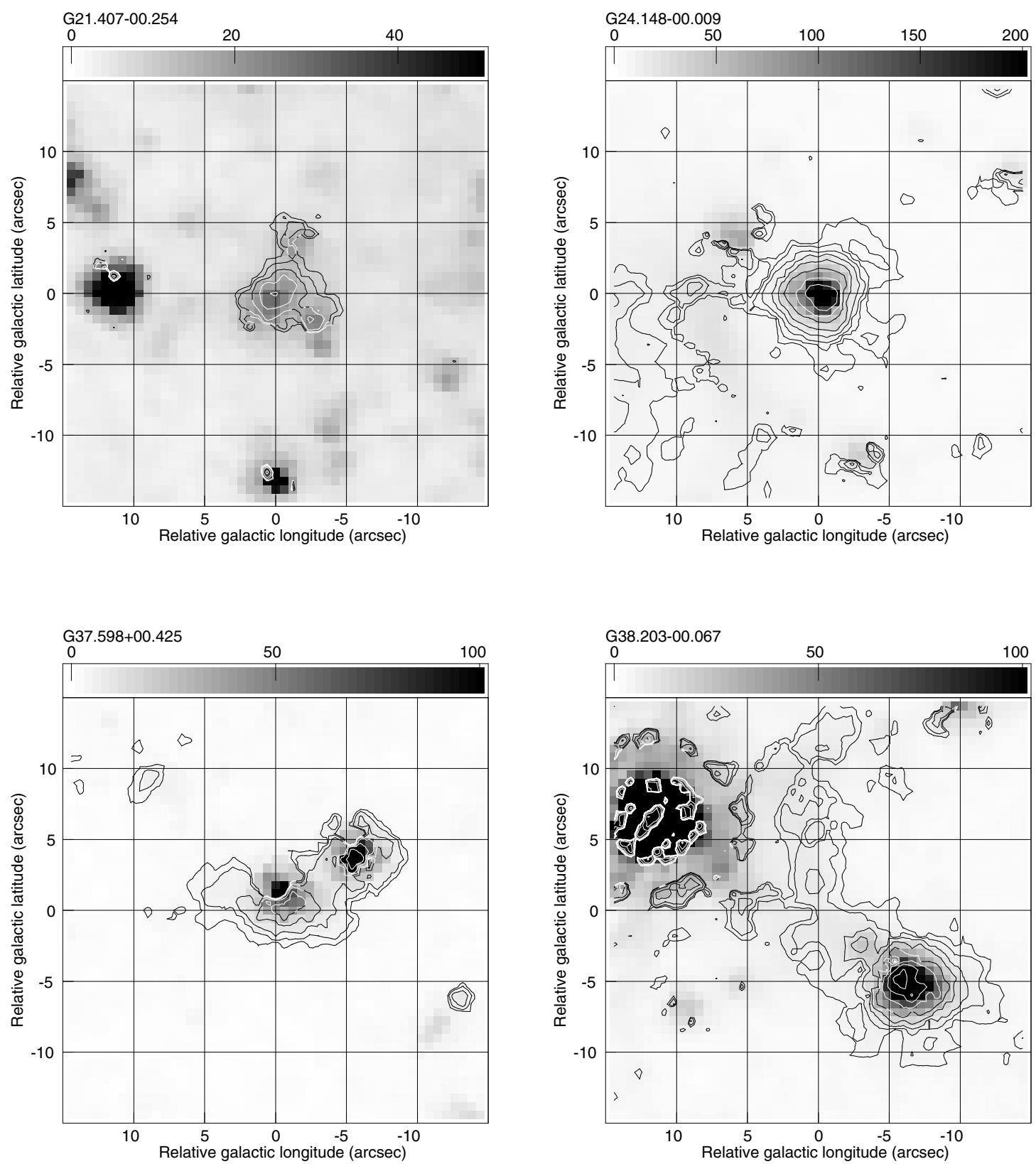

Fig. 6. Spitzer IRAC $4.5 \mu \mathrm{m}$ (grey) and $4.5 \mu \mathrm{m}-3.6 \mu \mathrm{m}$ (contour) images centered on selected methanol sources (at coordinates taken from Table 5). The grey scale in $\mathrm{MJy} \mathrm{sr}^{-1}$ is indicated in the horizontal bar and contour levels are $1 \mathrm{MJy} \mathrm{sr}^{-1} \times(1,2,4,8,16,32,64,128)$. The methanol maser G21.407-00.254 coincides exactly with the brightest pixel in the $4.5 \mu \mathrm{m}-3.6 \mu \mathrm{m}$ image, at the map origin, which is offset from the $4.5 \mu \mathrm{m}$ peak. There are at least two other nearby MIR sources with $4.5 \mu \mathrm{m}$ emission excesses. Note the absence of further sources with extended $4.5 \mu \mathrm{m}-3.6 \mu \mathrm{m}$ emission excess in this $30^{\prime \prime} \times 30^{\prime \prime}$ field. The maser source G24.148-00.009 coincides with the peaks of emission both at $4.5 \mu \mathrm{m}$ and for the $4.5 \mu \mathrm{m}-3.6 \mu \mathrm{m}$ excess. The maser $\mathbf{G 3 7 . 5 9 8}+\mathbf{0 0 . 4 2 5}$ is offset from the brightest $4.5 \mu \mathrm{m}$ emission. Note the highly asymmetric morphology of the extended $4.5 \mu \mathrm{m}-3.6 \mu \mathrm{m}$ excess emission and the coincidence of the maser emission with a maximum of this excess. In the case of G38.203-00.067, the maser emission does not coincide with either of the two brightest sources but is close ( $\left.\sim 1^{\prime \prime} .7\right)$ to a weak bump of $4.5 \mu \mathrm{m}-3.6 \mu \mathrm{m}$ excess located in a very extended $\operatorname{arc}\left(\sim 25^{\prime \prime}\right)$ of diffuse $4.5 \mu \mathrm{m}$ emission. Further inspection of IRAC images at $5.8 \mu \mathrm{m}$ suggests that this bump could be a weak MIR object. 